This article was downloaded by: [Princeton University Library]

On: 9 December 2009

Access details: Access Details: [subscription number 908309709]

Publisher Taylor \& Francis

Informa Ltd Registered in England and Wales Registered Number: 1072954 Registered office: Mortimer House, 3741 Mortimer Street, London W1T 3JH, UK

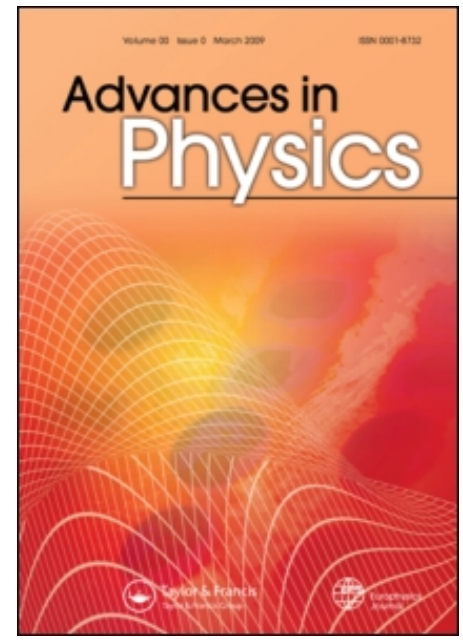

\title{
Advances in Physics
}

Publication details, including instructions for authors and subscription information:

http://www.informaworld.com/smpp/title content=t713736250

\section{Diffusion in disordered media}

Shlomo Havlin; Daniel Ben-Avraham

To cite this Article Havlin, Shlomo and Ben-Avraham, Daniel(2002) 'Diffusion in disordered media', Advances in Physics, 51: $1,187-292$

To link to this Article: DOI: $10.1080 / 00018730110116353$

URL: http://dx.doi.org/10.1080/00018730110116353

\section{PLEASE SCROLL DOWN FOR ARTICLE}

\footnotetext{
Full terms and conditions of use: http://www.informaworld.com/terms-and-conditions-of-access.pdf

This article may be used for research, teaching and private study purposes. Any substantial or systematic reproduction, re-distribution, re-selling, loan or sub-licensing, systematic supply or distribution in any form to anyone is expressly forbidden.

The publisher does not give any warranty express or implied or make any representation that the contents will be complete or accurate or up to date. The accuracy of any instructions, formulae and drug doses should be independently verified with primary sources. The publisher shall not be liable for any loss, actions, claims, proceedings, demand or costs or damages whatsoever or howsoever caused arising directly or indirectly in connection with or arising out of the use of this material.
} 


\title{
Diffusion in disordered media*
}

\author{
Shlomo Havlin广 and Daniel Ben-Avraham $†$ \\ $\uparrow$ Department of Physics, Bar-Ilan University, Ramat-Gan, Israel \\ $\ddagger$ Center for Polymer Studies, Boston University, Boston, MA 02215, USA
}

[Received 20 May 1987]

\begin{abstract}
Diffusion in disordered systems does not follow the classical laws which describe transport in ordered crystalline media, and this leads to many anomalous physical properties. Since the application of percolation theory, the main advances in the understanding of these processes have come from fractal theory. Scaling theories and numerical simulations are important tools to describe diffusion processes (random walks: the 'ant in the labyrinth') on percolation systems and fractals. Different types of disordered systems exhibiting anomalous diffusion are presented (the incipient infinite percolation cluster, diffusion-limited aggregation clusters, lattice animals, and random combs), and scaling theories as well as numerical simulations of greater sophistication are described. Also, diffusion in the presence of singular distributions of transition rates is discussed and related to anomalous diffusion on disordered structures.
\end{abstract}

\section{Contents}

PAGE

1. Introduction

1.1. Fractals

190

1.2. Random walks

2. Anomalous diffusion on fractals

2.1. Diffusion on the Sierpinski gasket

2.2. Conductivity and diffusion: the Einstein relation

2.3. Fracton (spectral) dimensionality and the Alexander-Orbach conjecture

2.4. Probability densities

3. Diffusion on percolation clusters 204

3.1. Analogy with diffusion on fractals 205

3.2. Two ensembles 206

3.3. Scaling theory 207

3.4. Chemical distance metric 209

3.5. Probability densities and multifractals 214

$\begin{array}{ll}\text { 3.6. Numerical results } & 218\end{array}$

$\begin{array}{ll}\text { 3.7. Continuum percolation } & 220\end{array}$

4. Diffusion on loopless structures 224

4.1. Relation between transport exponents and structural exponents 225

4.2. Diffusion on branched polymers modelled by lattice animals 225

4.3. Diffusion on DLA clusters 227

4.4. Diffusion on comb-like structures 229

* This article was originally published in Advances in Physics, volume 36, 1987. It had attracted 811 citations by October 2001, and is ranked 18 in the index of articles attracting more than 100 citations. 
5. Diffusion on random and hierarchical structures

5.1. Transport in one-dimensional systems with a power-law distribution of conductivities

5.2. One-dimensional systems with a power-law distribution of potential barriers or wells and hierarchical structures

5.3. Transport in strips $(n \times \infty)$ and in $d \geqslant 2$ with random barriers and wells

5.4. Potential barriers and wells on fractals

5.5. Anomalous diffusion on combs and fractals

6. Biased diffusion in disordered media 246

6.1. Dynamical phase transition 247

6.2. Bias leading to logarithmic behaviour $\quad 250$

6.3. Biased diffusion on percolation clusters 251

6.4. Bias along the backbone 255

$\begin{array}{ll}\text { 6.5. Time-dependent fields } & 257\end{array}$

7. Trapping and diffusion-limited reactions 258

$\begin{array}{ll}\text { 7.1. Trapping } & 259\end{array}$

7.2. One-species annihilation and the effect of diffusion 261

7.3. Two-species annihilation and the effect of spatial fluctuations in concentration

$\begin{array}{ll}\text { 8.1. Diffusion of particles with hard-core interactions } & 265 \\ \text { 8.2. Diffusion on deterministic fractals } & 267\end{array}$

$\begin{array}{ll}\text { 8.3. Self-avoiding walks on fractals } & 270\end{array}$

8.4. Diffusion in other types of disordered systems 274

$\begin{array}{ll}\text { Acknowledgements } & 275\end{array}$

Appendix: Numerical methods $\quad 275$

A.1. Monte Carlo methods 275

$\begin{array}{ll}\text { A.2. Exact enumeration } & 277\end{array}$

A.3. The 'blind ant' and the 'myopic ant' 278

$\begin{array}{ll}\text { Notes added in proof } & 279\end{array}$

$\begin{array}{ll}\text { References } & 283\end{array}$

\section{Introduction}

The problem of diffusion in disordered media is part of the general problem of transport in disordered media. The range of applicability and of physical interest is enormous [1-28]. Most of the materials encountered in nature in everyday experience are non-crystalline, disordered materials. The classic theories of transport valid for crystals do not apply, and the physics of transport, and in particular of diffusion, is anomalous in these disordered systems. It is impossible to present an exhaustive list of the physical phenomena related to the subject reviewed in this article. Some typical examples are the problems of the transport properties in fractured $[2,29]$ and in porous [30] rocks, the anomalous density of states in randomly diluted magnetic systems [31, 32], in silica aerogels [33] and in glassy ionic conductors [34], anomalous relaxation phenomena in spin glasses [35] and in macromolecules [36], conductivity of superionic conductors such as hollandite [37] and of percolation clusters of $\mathrm{Pb}$ on thin films of $\mathrm{Ge}$ or $\mathrm{Au}$ [38-40], and of diffusion-controlled fusion of excitations in porous membrane films, polymeric glasses and isotropic mixed crystals [41], to mention a number of examples.

Much of our understanding of disordered systems comes from percolation theory. The percolation model has proved to be relevant and useful for the study of most disordered media. Since it is essentially a simple purely geometrical model, it 
has as much appeal to the mathematician as to the physicist. This subject is excellently reviewed in references [16-22]. Another important advance came with the advent of fractals $[23,24]$ and with the realization that fractals are a very good model for the geometrical structure of most disordered materials, as well as for the clusters generated by the percolation model itself. It is not our intention to review fractal theory (a complete presentation of the subject is given in references [23-28]). Rather, we present in section 1.1 only the definitions of the most basic concepts used in this review.

Diffusion and transport properties on both percolation and fractals have been extensively studied. The analysis of transport in disordered media by means of diffusion on percolation clusters was suggested by de Gennes [42]. The idea was to perform random walks on a percolation system, for which de Gennes coined the term 'ant in the labyrinth'. This can be used to measure the diffusion constant and to calculate the conductivity of the system via the Einstein relation for diffusion and conductivity. Numerical simulations of random walks on percolation clusters were carried out by Mitescu et al. [43]. With the recognition that fractal lattices are a good model for disordered systems, the problem of the ant in the labyrinth, originally intended for percolation, was extended to fractals. The study of finitely ramified fractals (see section 1.1) yielded rigorous results [44] for anomalous transport in disordered media.

The theory of random walks has been applied in many areas of science, especially as a model for transport phenomena. In uniform Euclidean systems, the meansquare displacement of a random walker, $\left\langle R^{2}(t)\right\rangle$, is proportional to the time $t$, $\left\langle R^{2}(t)\right\rangle \propto t$, for any number of spatial dimensions $d$ (Fick's law). However, in disordered systems, this law is not valid in general. Rather, the diffusion law becomes anomalous [45-49]:

$$
\left\langle R^{2}(t)\right\rangle \sim t^{2 / d_{\mathrm{w}}}
$$

with $d_{\mathrm{w}}>2$. This slowing down of the transport is caused by the delay of the diffusing particles in the dangling ends, bottlenecks and backbends existing in the disordered structure. Examples of disordered systems for which anomalous diffusion has been observed are the incipient infinite percolation cluster [45-49], diffusionlimited aggregation (DLA) clusters [50], lattice animals [51, 52], and the Sierpinski gasket [47] and other fractal lattices [53].

In addition to percolation, fractals and simple random walks, other models have been applied to the study of the anomalous transport properties of disordered media. Most notably, disordered systems have been modelled by regular lattices with a random distribution of transition rates or of bond conductivities. This approach is excellently reviewed by Alexander et al. [4]. On the level of random-walk theory, the simple random-walk model has been generalized to the multi-state random-walk [54] and to the continuous-time random-walk (CTRW) [55] models. Many of the anomalous transport results of disordered systems are reproduced by these models. For recent reviews on random-walk theory, including the multi-state random walk and the CTRW, see references [3, 14, 15]. The review of Haus and Kehr [3] contains a complete discussion of the use of CTRWs for the understanding of transport in disordered media. Because of the existing reviews of the approaches discussed above, the emphasis of the present review is on the analysis of the anomalous transport properties of disordered media using simple random-walk and scaling theory, 
applied to the percolation and, especially, to the fractal models of disordered structures.

\subsection{Fractals}

We begin with the definitions of the most basic properties of fractals. Fractals are mathematical objects with a Hausdorff-Besicovitch dimension that is not an integer. A fractal is not smooth at every point-it was this property, of being fractured at every point, that led Mandelbrot [23] to coin the term fractals (from the Latin participle: fractus). Some examples [23] of well-known fractals are pictured in figures 1,2 and 3. Fractals are best constructed in a recursive way. Thus, for example, the Koch curve (figure 1) is constructed by starting with a unit segment. The middle third section of this segment is erased and replaced by two other segments of equal length $\frac{1}{3}$. Next, the same procedure is repeated for each of the four resulting segments (of length $\frac{1}{3}$ ). The process is iterated ad infinitum. The limiting curve is of infinite length, yet it is confined to a finite region of the plane. The best way to characterize it

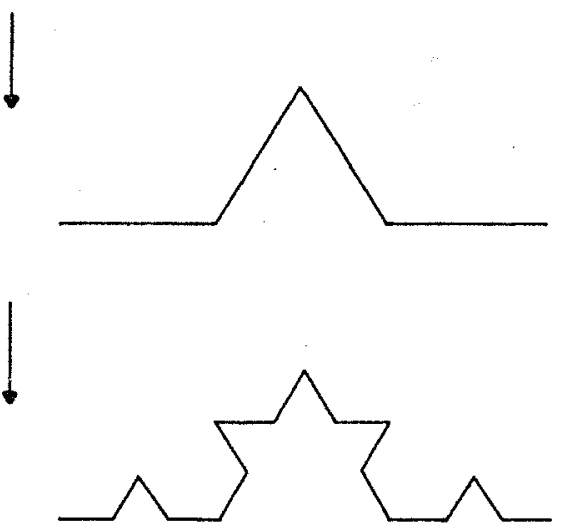

(a)

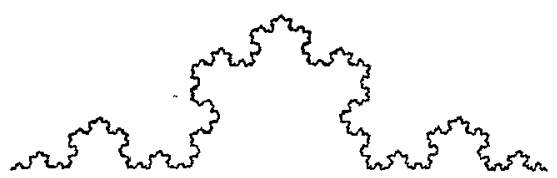

(b)

Figure 1. Koch curve: (a) the iteration process by which it is constructed; $(b)$ selfsimilarity - the central 'snowman' is surrounded by two exact copies of itself.

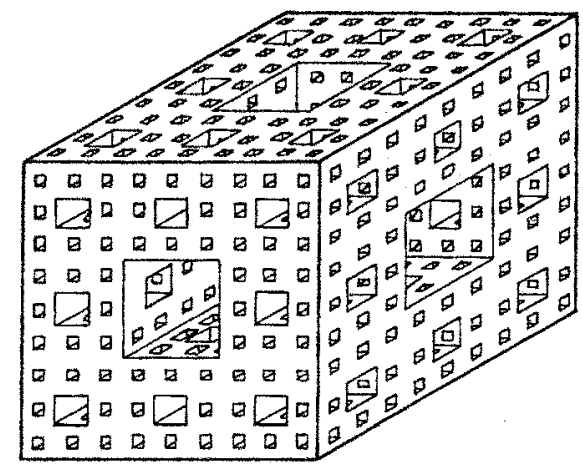

Figure 2. The Sierpinski sponge. 


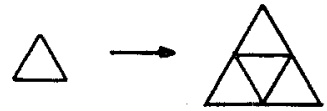

(a)

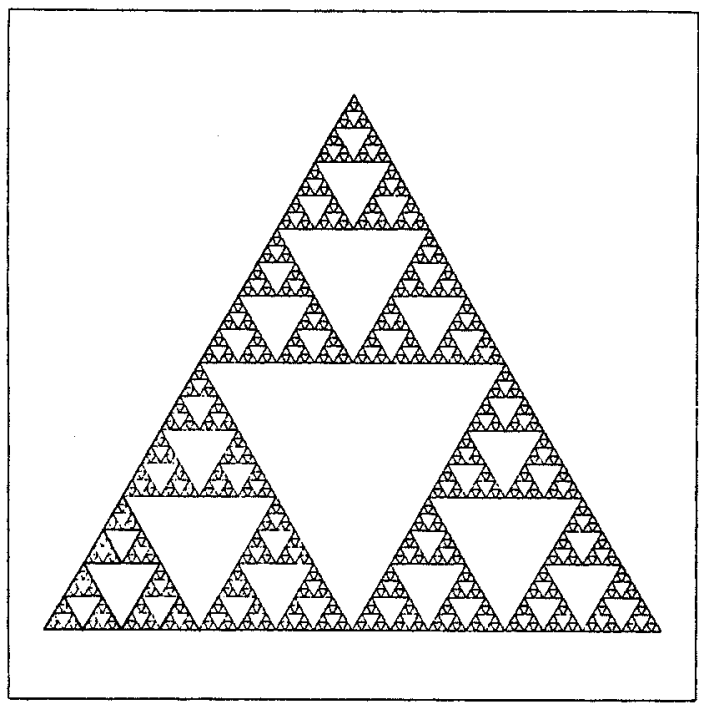

(b)

Figure 3. Sierpinski gasket in $d=2$ : (a) the iteration process from a triangular genus; (b) a Sierpinski gasket drawn to the sixth generation.

is by using its Hausdorff-Besicovitch or fractal dimension, $d_{\mathrm{f}}$. In a Koch curve magnified by a factor of three there fit exactly four of the original curves. Therefore its fractal dimension is given by $3^{d_{\mathrm{f}}}=4$, or $d_{\mathrm{f}}=\ln 4 / \ln 3=1.262$.

When calculating $d_{\mathrm{f}}$, we notice another striking property of the Koch curve- the property of self-similarity. Indeed, if we examine the Koch curve, or the Koch snowflake, as it is frequently called, we notice that there is a central object in the figure that is reminiscent of a snowman. To the right and to the left of this central snowman, there are two other snowmen, each being an exact reproduction only smaller by a factor of $\frac{1}{3}$. Each of the smaller snowmen has in turn two still smaller copies of themselves to their right and left, etc.

The Sierpinski sponge of figure 2 is constructed by starting from a cube, subdividing it into $3 \times 3 \times 3=27$ smaller cubes, and taking out the central small cube and its six nearest neighbours. Each of the remaining 20 small cubes is processed in the same way, and the whole procedure is iterated ad infinitum. After each iteration, the volume of the sponge is reduced by a factor of $\frac{20}{27}$, while the total surface area increases. In the limit of the fractal sponge, the surface area is infinite, while the volume vanishes. This is consistent with the fractal dimension of the Sierpinski sponge, which is given by $3^{d_{\mathrm{f}}}=20$, or $d_{\mathrm{f}}=\ln 20 / \ln 3=2.727$. Perhaps the most famous example of a fractal is the Sierpinski gasket of figure 3. It is constructed from an equilateral triangle, subdividing it into four smaller triangles and taking out the central triangle. This generator is iterated ad infinitum. The resulting Sierpinski gasket has a fractal dimension given by $2^{d_{\mathrm{f}}}=3$ or $d_{\mathrm{f}}=$ $\ln 3 / \ln 2=1.585$.

The great attraction of the Sierpinski gasket is that many physical problems involving it can be solved exactly. This is due to the self-similarity property and to the fact that the Sierpinski gasket is finitely ramified. A fractal is finitely ramified $[24,44]$ if any bounded subset of the fractal can be isolated by cutting a finite 
number of bonds, interactions or sites. Thus the Sierpinski gasket and the Koch curve are finitely ramified, but the Sierpinski sponge is infinitely ramified. These two properties, of self-similarity and of being finitely ramified, enable an exact renormalization-group approach for most physical problems considered on the Sierpinski gasket.

The fractal dimension $d_{\mathrm{f}}$ and ramification, finite or infinite, are just two of the parameters characterizing fractals. Many other exponents and characterization s are needed in order to emphasize other aspects and properties of fractals. Thus, for example, the concept of lacunarity $[23,24,56]$ is related to the degree of homogeneity of a fractal and to the extent that it is translation-invariant. The fracton [45] or spectral [49] dimension $d_{\mathrm{s}}$ is an exponent describing the scaling of the density of states with the energy on a fractal. The chemical exponent $\tilde{\nu}$ [57] describes the scaling of the number of bonds connecting two points on a fractal along the minimal or chemical path between them. Some of these exponents and concepts are used in the text. Their exact definition and a more detailed explanation will be presented when needed (see also table 9).

All of the above examples of fractals are deterministic fractals, meaning fractals that are constructed by a rigorous deterministic recursive law. There are, however, many other objects that have the self-similarity property characteristic of fractals, but only in a statistical sense. For these objects a fractal dimension $d_{\mathrm{f}}$ is still easily defined by the scaling of their mass $M$ with their linear size $L$ :

$$
M \sim L^{d_{\mathrm{f}}} .
$$

It was first realized by Mandelbrot [23] that many objects in nature are of a statistical-fractal shape. These range from polymers and coastlines to clouds and the pockmarked surface of the Moon. There is experimental evidence [58-62] that some real aggregates, such as gold colloids and silica-colloid aggregates, have a fractal structure (figure 4). A number of fractal models have been applied to physical problems; diffusion-limited aggregation [63] has been used as a model for real aggregates [8], dielectric breakdown [64], growth processes [11], viscous fingering $[30,65,66]$ and snowflakes (J. Nittman and H. E. Stanley, preprint); percolation theory $[18,19]$ is being used as a model for gelation [10], porous media and fractures [2]; and self-avoiding walks [67, 68] or other fractals [69, 70] are being used in modelling polymers [71]. These subjects have been discussed in recent books, reviews and conference proceedings [23-28].

\subsection{Random walks}

Throughout this review, we refer to a simple random walk on a lattice as model for diffusion [72]. In a simple discrete random walk the walker advances one step in unit time. Each step is taken to a nearest neighbour of the site. In a disordered system, such as a percolation cluster, not all of the nearest neighbours belong to the substrate. The simple random walker can step with equal probability to any of the nearest neighbour sites that belongs to the substrate. As a matter of fact, there are several ways of assigning transition probabilities for stepping from site to site. Some of the most common and useful methods are summarized in section A.3 of the appendix.

One of the most important physical parameters describing a random walk is the mean-square displacement $\left\langle R^{2}(t)\right\rangle$ covered by the walker after having stepped $t$ steps (or, equivalently, after a time $t$ ). Denote the walker steps by $\mathbf{u}_{1}, \mathbf{u}_{2}, \ldots, \mathbf{u}_{t}$. Then 


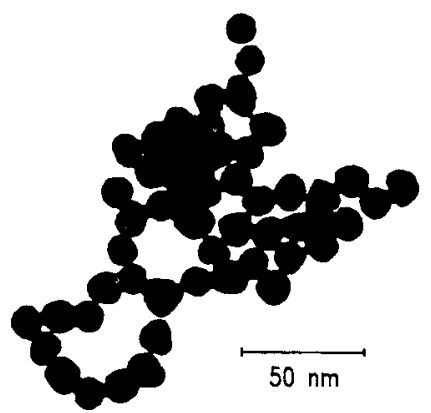

(a)

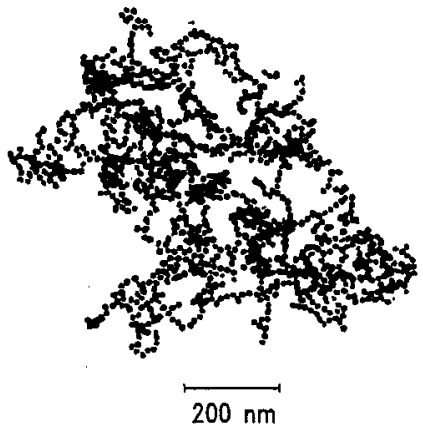

(c)

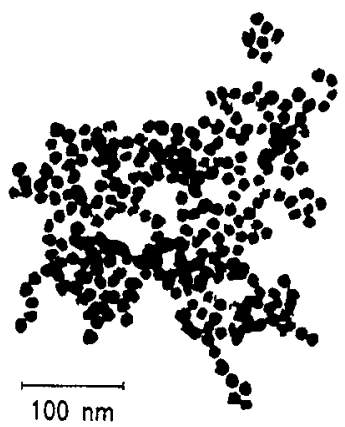

(b)

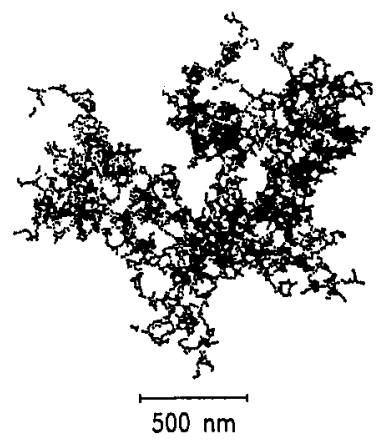

(d)

Figure 4. Example of gold-colloid aggregate made by Weitz and Oliveria [61]. Direct analysis in terms of (1.2) gives $d_{\mathrm{f}}=1.75$.

$$
\left\langle R^{2}(t)\right\rangle=\left\langle\left(\sum_{i=1}^{t} \mathbf{u}_{i}\right)^{2}\right\rangle=t+2 \sum_{i>j}^{t}\left\langle\mathbf{u}_{i} \cdot \mathbf{u}_{j}\right\rangle .
$$

For normal Euclidean space the correlations $\left\langle\mathbf{u}_{i} \cdot \mathbf{u}_{j}\right\rangle$ are all zero. Thus for Euclidean space one has the usual result for normal diffusion that $\left\langle R^{2}(t)\right\rangle=t$.

A simple random walk is statistically self-similar. To see this, consider the random walk as it looks when one regards $n$ consecutive steps as one single superstep. Each of the supersteps is a random jump $\mathbf{r}$ from the present site of the walker to a neighbouring site. The random supersteps are distributed according to a probability function $P_{n}(\mathbf{r})$. In the limit that $n \gg 1, P_{n}(\mathbf{r})$ tends to a Gaussian distribution. This is a simple result of the Central-Limit Theorem. It is evident that, statistically, the same random walk results for different values of $n$, provided that $n$ is large enough. The only difference between walks with $n=n_{1}$ and with $n=n_{2} \neq n_{1}$ is that in the first case a step takes a time $\tau_{1}=n_{1}$, whereas in the second case the time needed is $\tau_{2}=n_{2}$. Also, the average length of a step is $n_{1}^{1 / 2}$ in the first case and $n_{2}^{1 / 2}$ in the second case. This means that if we scale time as $t \rightarrow \lambda t$ and length as $r \rightarrow \lambda^{1 / 2} r$ then two walks with $n_{2}=\lambda n_{1}$ will be exactly equivalent under this scaling. Hence the simple random walk is statistically self-similar. Another consequence is that the simple random walk is a statistical fractal. Upon dilation of space by a factor of $\lambda^{1 / 2}$, the number of steps (or the 'mass' of the walk) increases by a factor of $\lambda$. Therefore the fractal dimension of a random walk is $d_{\mathrm{w}}=\ln \lambda / \ln \lambda^{1 / 2}=2$. It is a curious result 
that random walks performed on disordered but statistically self-similar structures are still self-similar themselves, exactly as in Euclidean space. The only difference is that the usual diffusion exponent, $d_{\mathrm{w}}=2$ is no longer equal to 2 . Thus diffusion becomes anomalous.

The outline of the review is as follows. In section 2 the Sierpinski gasket is used as an example to define and to calculate various concepts related to transport properties. In percolation clusters above criticality, the classical phonons found at length scales greater than the correlation length $\xi$ give way to fractons, which are excitations in length scales smaller than $\xi$. Also included in section 2 is a discussion of fractons and of the Alexander-Orbach [45] conjecture that the related fracton dimensionality $d_{\mathrm{s}}$ is $\frac{4}{3}$ for percolation in any dimension.

Transport properties of percolation clusters, a model for disordered media, are reviewed in section 3. The topics covered include scaling theories (which relate the diffusion exponents to the conductivity exponents), the chemical distance (which is the shortest path on a structure connecting two sites and is a natural metric when dealing with diffusion) and numerical results.

For loopless structures some of the long-range correlations (associated with loops) do not exist. This greatly simplifies the study of such structures, enabling one to obtain an exact relation between dynamical transport exponents and static geometrical exponents. Such a relation has not yet been found for structures with loops, such as percolation clusters. 'Random combs', i.e. comb-like structures with a random distribution of the length of their teeth, are exactly solved for their transport properties. A distribution of lengths of the teeth can be replaced by a distribution of transition probabilities from site to site of a random walker on a (one-dimensional) line. Random combs are used to model transport phenomena on fractals and shed light on the problem posed by the more complex structures. These subjects are reviewed in sections 4 and 5 .

A bias field applied to a diffusing particle in a disordered medium has two opposite effects. On the one hand, the particles are pushed in the direction of the field, giving rise to a drift velocity. On the other hand, the field traps the particles in the dangling ends: in order to exit a dangling end, the particle must move against the field. These two opposite effects give rise to a dynamical phase transition. For a field strength $E$ smaller than a critical value $E_{\mathrm{c}}$, the particles experience a finite drift velocity. This velocity vanishes for $E>E_{\mathrm{c}}$, and anomalous diffusion takes place, with a field-dependent critical exponent. The dynamical phase transition is predicted from the exact solutions of biased diffusion on a comb, and is obtained numerically in simulations of biased diffusion on percolation. Biased diffusion is reviewed in section 6 .

Section 7 contains a discussion of the problems of trapping of diffusing particles in the presence of random traps and of diffusion-controlled reactions. Finally, several miscellaneous topics are covered in section 8 , such as diffusion of particles with hard-core interactions, diffusion on deterministic fractals, and self-avoiding walks on fractals.

Finally, a word is in order about the citing of references. For many topics, the number of references is so large that we have chosen to cite representative articles rather than to attempt to be exhaustive. No implication is intended of the importance of articles cited relative to articles not cited. We apologize in advance for those cases where our selection was clearly faulty. 


\section{Anomalous diffusion on fractals}

In this chapter we discuss various concepts related to diffusion in disordered media. Anomalous diffusion is most easily analysed on exact fractal lattices with a finite ramification, for which many of its properties can be calculated exactly [24, 44, 73]. It is convenient to use diffusion on the Sierpinski gasket (figure 3 ) as our major example. Although an exact fractal lattice is not a disordered medium, diffusion on a fractal exhibits anomalies similar to those present in disordered media. This is due to the obstacles, holes or traps, at all scales of length, appearing in both types of structures.

The fractal dimension of the $d=2$ Sierpinski gasket is $d_{\mathrm{f}}=\ln 3 / \ln 2$, since by magnifying the scale by a factor of 2 , the mass is seen to increase by a factor of 3 . For the Sierpinski gasket in $d$ dimensions the fractal dimension is $d_{\mathrm{f}}=\ln (d+1) / \ln 2$. Note also the self-similarity of the Sierpinski gasket. On magnifying a small triangle of the Sierpinski gasket, which itself consists of an infinite hierarchy of smaller triangles, to the size of the whole gasket, both the magnified and the original pictures look exactly the same.

\subsection{Diffusion on the Sierpinski gasket}

To illustrate anomalous diffusion, imagine a random walker on the Sierpinski gasket. At each step the walker chooses randomly to move to one of the (four) nearest-neighbour sites on the gasket. $\dagger$

We then look at the mean-square displacement after $N$ steps, $\left\langle R_{N}^{2}\right\rangle$. Because of the self-similarity of random walks in Euclidean lattices [23], we expect the walk on the Sierpinski gasket to be self-similar as well. Thus for large $N$

$$
\left\langle R_{N}^{2}\right\rangle \sim N^{2 / d_{\mathrm{w}}}
$$

where $d_{\mathrm{w}}$ is the anomalous-diffusion exponent. Note that $d_{\mathrm{w}}$ is in fact the fractal dimensionality of the path of the random walker on the gasket [74].

A straightforward way to calculate $d_{\mathrm{w}}$ is to apply the exact enumeration method (see section A.2 of the appendix), in which the square displacements $R^{2}$ of all possible random walks starting from a common origin are averaged. This yields the exact result for $\left\langle R^{2}\right\rangle$. A plot of $\ln \left\langle R_{N}^{2}\right\rangle^{1 / 2}$ as a function of $\ln N$ is presented [47] in figure 5. It is evident from the plot that after a transient of about ten steps the meansquare displacement follows a power law with an anomalous-diffusion exponent $d_{\mathrm{w}}=2.32 \pm 0.01$. We note that this result is independent of the origin, although in general (2.1) implies an averaging over different origins as well as on the walks emanating from each.

There exist some simple renormalization schemes $[44,75,76]$ for the calculation of the exact value of $d_{\mathrm{w}}$ for the gasket. Consider, for example the mean transit time $T$ needed to traverse a gasket unit from one of its vertices to one of the remaining vertices $O$ (figure $6(a)$ ) [76]. One can then calculate the corresponding time $T^{\prime}$ for

$\dagger$ It has been proved by S. Goldstein (preprint, 1987) that diffusion in the Sierpinski gasket (a Cantor set of Lebesgue measure zero) is the limiting case of a (discrete) random walk on the 'Sierpinski lattice'. The Sierpinski lattice is the object obtained by iterating the genus of figure $1(a)$ a finite number of times. Thus the smallest length scale in the Sierpinski lattice is the length of the bonds of the genus. In contrast, the Sierpinski gasket has no finite length associated with it. Because of the equivalence of the gasket and the lattice we do not make a distinction between the two. 


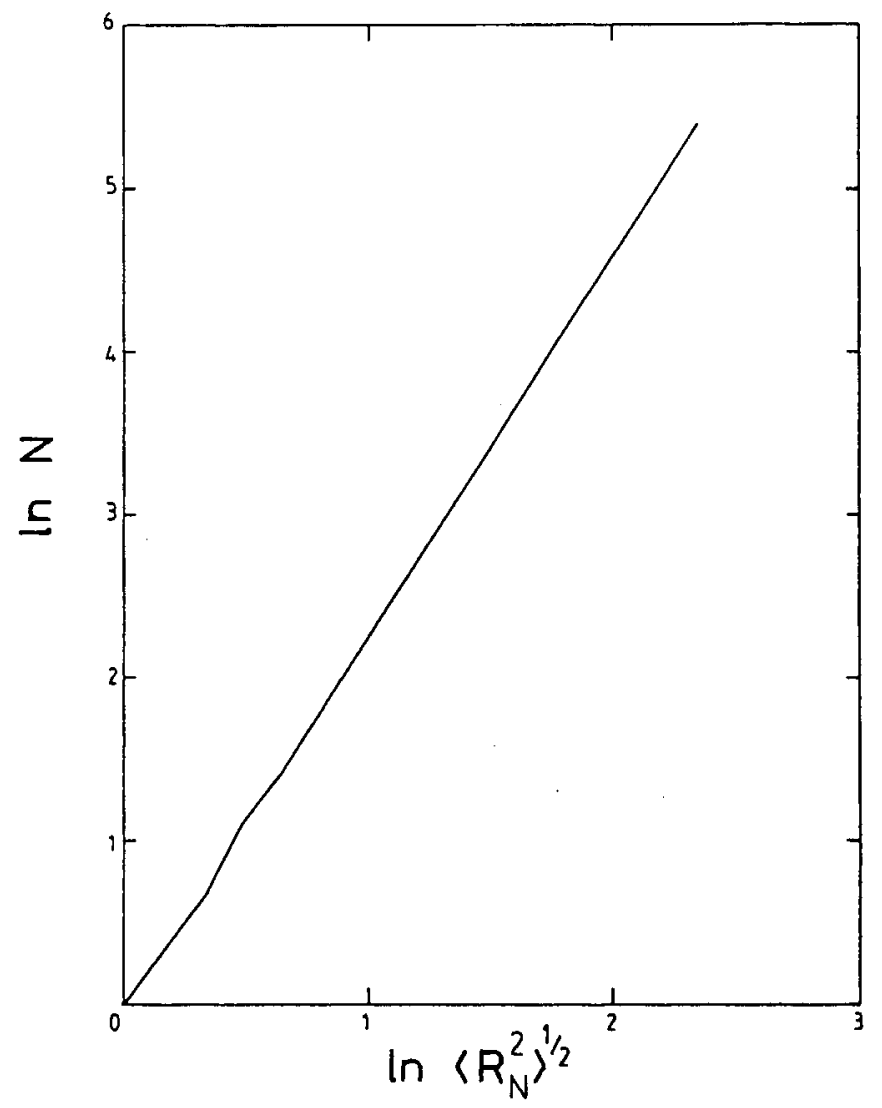

Figure 5. Plot of $\ln N$ as a function of $\ln \left\langle R_{N}^{2}\right\rangle^{1 / 2}$ on a Sierpinski gasket using the exactenumeration method.

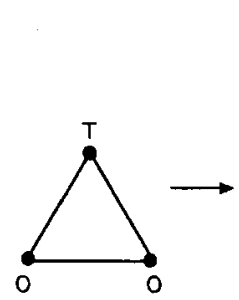

(a)

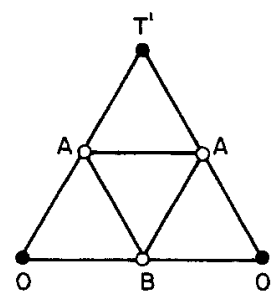

(b)

Figure 6. Rescaling of transit time for traversing the gasket. The walker enters the gasket at the top vertex and $(a)$ takes a time $T$ to exist through the lower $O$ vertices. $(b)$ The rescaled gasket, $T \rightarrow T^{\prime}$ and $A$ and $B$ are transit times from the internal (decimated) vertices to the lower $O$-vertices.

exiting a rescaled fractal unit by a factor of two (figure $6(b)$ ). This is done by exploiting the Markov property of the random walk on the fractal. Thus $T^{\prime}$ equals the time $T$ to exit the first gasket unit, plus $A$, the mean transit time needed to leave the rescaled unit from then on. Using the same reasoning for the times $A$ and $B$ (the mean exit times starting from the decimated internal vertices), one gets 


$$
\left.\begin{array}{l}
T^{\prime}=T+A \\
4 A=4 T+A+B+T^{\prime} \\
4 B=4 T+2 A .
\end{array}\right\}
$$

The solution is $T^{\prime}=5 T$ (and $A=4 T, B=3 T$ ), which is the rescaling of time for a diffusion process on the gasket, upon the rescaling of length by a factor of two. It follows that $d_{\mathrm{w}}=\ln 5 / \ln 2=2.322$. Note the agreement with the result of the exact enumeration presented earlier. This renormalization scheme is easily generalized for the $d$-dimensional Sierpinski gasket, yielding for the analogous transit times

$$
\left.\begin{array}{rl}
T^{\prime} & =T+A, \\
2 d A & =2 d T+(d-1) A+(d-1) B+T^{\prime}, \\
2 d B & =2 d T+2 A+(2 d-4) B,
\end{array}\right\}
$$

with the solution $T^{\prime}=(d+3) T$ (and $\left.A=(d+2) T, B=(d+1) T\right)$ and $d_{\mathrm{w}}(d)=$ $\ln (d+3) / \ln 2$. This result can be derived by means of a calculation of the resistance exponent for the gasket done by Gefen et al. [44] (see the next section).

\subsection{Conductivity and diffusion: the Einstein relation}

One of the main reasons for the interst in diffusion in disordered media is its immediate relation to the conductivity of the medium. The relation between diffusion and conductivity is established $\dagger$ via the Einstein relation

$$
\sigma_{\mathrm{dc}}=\frac{e^{2} n}{k_{\mathrm{B}} T} D,
$$

where $\sigma_{\mathrm{dc}}$ is the d.c. conductivity, $n$ is the carrier density and $D$ is the diffusion constant

$$
D \equiv\left\langle R_{N}^{2}\right\rangle / N, \quad N \gg 1 .
$$

In the present context, the carrier density $n$ means the density of the substrate. This scales with length $R$ as $n \sim R^{d_{\mathrm{f}}-d}$, where $d_{\mathrm{f}}$ is the fractal dimension of the substrate. The conductivity exponent $\tilde{\mu}$ is defined by the scaling of the conductivity $\sigma_{\mathrm{dc}} \equiv \sigma$ with length $R$ :

$$
\sigma(R) \sim R^{-\tilde{\mu}}
$$

Using (2.1) and (2.5), one obtains from the Einstein relation [45]

$$
N \sim R^{2-d+\tilde{\mu}+d_{\mathrm{f}}} \equiv R^{d_{\mathrm{w}}},
$$

or

$$
d_{\mathrm{w}}=2-d+d_{\mathrm{f}}+\tilde{\mu} \equiv d_{\mathrm{f}}+\tilde{\zeta}
$$

In the last equation we have used the resistance exponent $\tilde{\zeta}$, defined by the scaling of resistance with length $\varrho(R) \sim R^{\tilde{\zeta}}$ (the relation between resistance and conductivity of a bulk sample is such that $\tilde{\zeta}=2-d+\tilde{\mu})$.

Returning to the example of the Sierpinski gasket, we mention that $\tilde{\mu}$ or $\tilde{\zeta}$ are easily calculated by an exact renormalization procedure [44]. In $d$ dimensions

$\dagger$ Y. Gefen and I. Goldhirsch (preprint, 1987), studied the question whether the Einstein relation holds for disordered random media (see also references [77, 78]). They find that it holds, with a redefinition of time. This time conversion is of course irrelevant for the d.c. conductivity. 
$\tilde{\zeta}=\ln [(d+3) /(d+1)] / \ln 2$. Combining the fractal dimensionality of the gasket $d_{\mathrm{f}}=\ln (d+1) / \ln 2$ with $(2.7 b)$ and the above result reproduces the result $d_{\mathrm{w}}=\ln (d+3) / \ln 2$, which was derived earlier in this section.

The usefulness of diffusion for the conductivity problem is now evident. From $(2.7 \mathrm{~b})$, we can see that it is possible to obtain the value of the conductivity exponent $\tilde{\mu}$ from a knowledge of the fractal dimension and the anomalous diffusion exponent of the medium. Until this method for measuring conductivity was discovered, the alternative was a direct calculation of the conductivity, involving the solution of Kirchhoff's equations for the specific disordered substrate under discussion. The complications involved limited calculations to samples of moderate size, even then requiring shortcuts and some very sophisticated algorithms [79]. As a result, there was a large uncertainty, for example, concerning the numerical value of $\tilde{\mu}$ for percolation clusters. In contrast, the evaluation of $d_{\mathrm{w}}$ is easily carried out by either a Monte Carlo simulation, or by an exact enumeration procedure. The fractal dimensionality of the substrate, which is also required for the calculation of $\tilde{\mu}$, $(2.7 \mathrm{~b})$, can be derived from the diffusion problem as well. It is related to the probability of the random walker returning to the origin (details are presented in the next section). It can also be obtained by a straightforward geometrical measurement based on its definition. In summary, $(2.7 \mathrm{~b})$ provides us with a highly accurate yet easy method of measuring the conductivity exponent. This was used to obtain reliable estimates of $\tilde{\mu}$ for percolation clusters [80-83], lattice animals [51, 52], diffusion-limited aggregates [50, 84], and other random substrates [85-87].

Another efficient numerical method for calculating the conductivity exponent of random resistor networks is the transfer-matrix technique of Derrida and Vannimenus [88]. Technical details of this method as well as a computer program for three-dimensional percolation systems have been given in reference [89]. This method has been used by Zabolitzky [90] to obtain accurate estimates of the conductivity exponent in two-dimensional percolation. For more details of numerical results in percolation see section 3 .

\subsection{Fracton (spectral) dimensionality and the Alexander-Orbach conjecture}

The problem of diffusion is closely related to the density of states [91] in the substrate. This can be best understood from the relation [4, 92] between the density of states $\mathscr{N}(\epsilon)$ and the probability $P(0, N)$ of the random walker returning to the origin

$$
P(0, N)=\int_{0}^{\infty} \mathscr{N}(\epsilon) \exp (-\epsilon N) \mathrm{d} \epsilon
$$

After performing $N$ steps, the number of sites a random walker has visited is proportional to the volume $R^{d_{\mathrm{f}}} \sim N^{d_{\mathrm{f}} / d_{\mathrm{w}}}$. Therefore the probability of returning to the origin scales as [45]

$$
P(0, N) \sim 1 / R^{d_{\mathrm{f}}} \sim N^{-\mathrm{d}_{\mathrm{f}} / d_{\mathrm{w}}} .
$$

Using this result in $(2.8 a)$, one obtains the following expression for the density of states on the substrate:

$$
\mathscr{N}(\epsilon) \sim \epsilon^{d_{\mathrm{f}} / d_{\mathrm{w}}-1} \equiv \epsilon^{d_{\mathrm{s}} / 2-1} .
$$

This is similar to the usual expression for Euclidean space, $\mathscr{N}(\epsilon) \sim \epsilon^{d / 2-1}$, except that $d$ is replaced by $2 d_{\mathrm{f}} / d_{\mathrm{w}}$. The ratio $2 d_{\mathrm{f}} / d_{\mathrm{w}}$ is then identified as the relevant dimension for the density of states in a substrate, and is called fracton dimensionality 
[45] or spectral dimensionality [49]. We denote it by $d_{\mathrm{s}}$ ('s' for spectral) to avoid ambiguity with the fractal dimensionality $d_{\mathrm{f}}$. The fracton dimensionality also characterizes the number of distinct sites, $S(N)$, visited by the random walker, as can be seen from $(2.8 b)$ :

$$
S(N) \sim N^{d_{\mathrm{s}} / 2}
$$

In recent years there has been much excitement about the Alexander-Orbach (AO) conjecture [45] that $d_{\mathrm{s}}=\frac{4}{3}$ for percolation clusters at criticality in any dimension above 1 . The fracton dimensionality is exactly $\frac{4}{3}$ in $d=6$, and is surprisingly close to $\frac{4}{3}$ for $1<d<6$, giving rise to this conjecture. Much theoretical [93-97] and numerical [82, 90, 98-101] effort has been made in the attempt to prove or disprove the AO conjecture. There have also been attempts to generalize the AO conjecture to other statistical fractals besides percolation $[50,93]$. It is now clear that it does not generalize to all statistical fractals. For example, the AO conjecture cannot hold simultaneously for percolation clusters and for the backbones of percolation clusters [102].

One of the many attempts to justify the AO conjecture was carried out by Rammal and Toulouse [49]. They suggest that the number of distinct sites $S(N)$ visited by a random walker after $N$ steps is related to the number of growth sites $G(N)$ (those sites with accessible neighbours out of $S(N)$ ) via the equation

$$
\frac{\mathrm{d} S(N)}{\mathrm{d} N} \sim \frac{G(N)}{S(N)} .
$$

The AO conjecture then follows if $G(N) / S(N) \sim[S(N)]^{-1 / 2} \sim R(N)^{-d_{\mathrm{f}} / 2}$, or $G(N) \sim R(N)^{d_{\mathrm{f}} / 2}$. It has been argued [95, 103] that $G(N)$ might scale as a simple sphere cut of the fractal substrate [24], leading to $G(N) \sim R(N)^{d_{\mathrm{f}}-1}$, which rules out the AO conjecture. One finds that the difference between $d_{\mathrm{f}} / 2$ and $d_{\mathrm{f}}-1$ for two-dimensional percolation (for which most of the numerical work is performed) is very small, making it difficult to reach a decision in this dispute. However, calculations have been performed [80] on an exact fractal lattice, similar to the Sierpinski gasket (figure 7) and the results show that (a) the Rammal-Toulouse equation (2.11) is closely satisfied and $(b)$ the front of the diffusion process does not scale as a spherical cut of the fractal substrate. Note that $(a)$ alone does not prove the AO conjecture, but (b) eliminates the doubt cast on it by the spherical-cut argument. Indeed, the plot of $\ln G(N)$ against $\ln S(N)$ (figure 8) for the fractal lattice in figure 7 shows that $G(N) \sim S(N)^{x}$, with $x=0.53 \pm 0.01$, in contrast with the

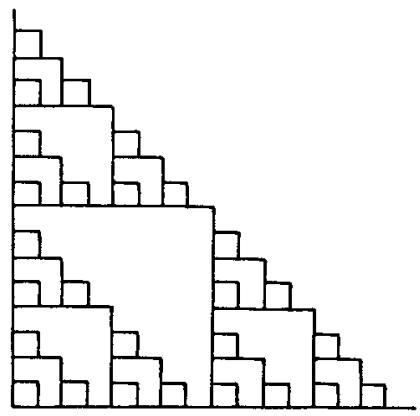

Figure 7. A fractal similar to the Sierpinski gasket. The critical exponents are the same as for the Sierpinski gasket. The fractal is shown to four generations. 


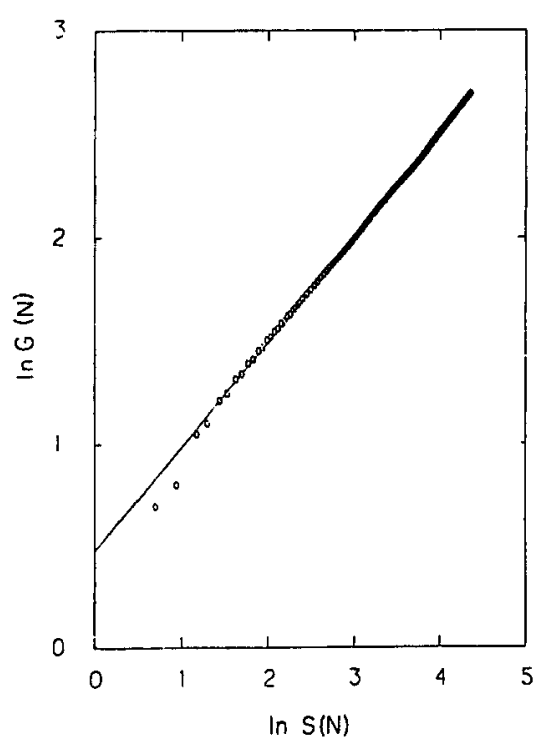

Figure 8. Plot of $\ln G(N)$ against $\ln S(N)$ for diffusion on the fractal described in figure 7. The circles represent the numerical data and the solid line is drawn from the prediction of the Rammal-Toulouse equation (2.11) [49].

value $x=\left(d_{\mathrm{f}}-1\right) / d_{\mathrm{f}}=0.369$ that is predicted by the assumption of a sphere cut. The value of $x$ obtained from figure 8 is consistent with the theoretical value, $x=2-2 / d_{\mathrm{s}}=0.535$ obtained from (2.11), thus supporting the Rammal-Toulouse equation. One can also argue against a spherical-cut front of diffusion based on (a) alone. If we accept (a), that the Rammal-Toulouse equation is valid, then $G(N) \sim R^{2 d_{\mathrm{f}}-d_{\mathrm{w}}}$. In general, this contradicts the spherical-cut assumption that $G(N) \sim R^{d_{\mathrm{f}}-1}$ (a specific example is the Sierpinski gasket for which $d_{\mathrm{w}}$ and $d_{\mathrm{f}}$ are exactly known - as previously shown). Exceptions are loopless fractal structures (section 4), for which the chemical metric coincides with the Pythagorian metric, $d_{l}=d_{\mathrm{f}}$. There $d_{\mathrm{w}}=1+d_{\mathrm{f}}$ and the front of diffusion is a spherical cut, without contradicting the fact that the Rammal-Toulouse equation holds. For a more detailed discussion of the front of diffusion on fractals see references $[95,96$, 101-103].

There is now a large body of evidence against the AO conjecture. It violates an $\epsilon$-expansion [95] around $d=6$. Enormous Monte Carlo simulations [82, 90, $98,99]$, seem to rule out the conjecture for two-dimensional percolation. The disagreement of the $\epsilon$-expansion with the conjecture proves that it does not hold for $d=6-\epsilon$ when $\epsilon$ is infinitesimally small. However, it could be true that $d_{\mathrm{s}}=\frac{4}{3}$ when $\epsilon$ becomes an integer. Thus there is no rigorous analytical disproof of the AO conjecture for percolation. Also, in spite of the impressive amount of numerical work disproving the conjecture, we consider it to be an open question whether or not it is valid for percolation. For numerical results supporting the AO conjecture see references $[100,104]$. In addition, there exists a very recent consistency argument supporting this conjecture, which uses the Ioffe-Regel criterion for localization of the fractons [105].

The great interest in the conjecture results not only from the universal value assigned to $d_{\mathrm{s}}$, but also from the fact that it provides a relationship between 
dynamic and static exponents. Indeed, by assuming $d_{\mathrm{s}}=\frac{4}{3}=$ constant at the exact relation

$$
d_{\mathrm{s}}=2 d_{\mathrm{f}} / d_{\mathrm{w}},
$$

one immediately obtains $d_{\mathrm{w}}$, characterizing the dynamic process of diffusion, in terms of $d_{\mathrm{f}}$, which describes the static geometrical scaling of the mass of the substrate. The question of the relationship between static and dynamic exponents has attracted considerable attention. The AO conjecture is one of many attempts, such as the de Gennes proposition [106], and the Skal and Shklovski model [107] to provide an answer to this question.

Finally, we note that $d_{\mathrm{s}}=2$ is a critical dimension for the problem of diffusion on fractals, analogous to $d=2$ for diffusion in Euclidean space. Indeed, for $d_{\mathrm{s}}>2$, (2.12) tells us that $d_{\mathrm{w}}<d_{\mathrm{f}}$, meaning that a random walk on such a substrate is no longer recurrent. There is therefore interest [49, 53] in exact fractal lattices with a tunable $d_{\mathrm{s}}$, which would enable us to study the transition at $d_{\mathrm{s}}=2$. In particular, diffusion on fractals with $d_{\mathrm{s}}>2$ has been almost completely neglected, primarily because of the lack of examples of fractal lattices with $d_{\mathrm{s}}>2$. In reference [53] such fractal lattices are suggested and bounds are given for the conductivity and diffusion exponents. However, no numerical study of diffusion on these lattices has been published. A serious study of diffusion on substrates with $d_{\mathrm{s}}>2$ is therefore desirable.

\subsection{Probability densities}

When dealing with diffusion or random walks, probability densities are of general interest and of great importance [14, 72, 108]. Consider for example the probability density $P(\mathbf{r}, t)$ of finding a random walker at $\mathbf{r}$ at time $t$, having started at the origin at $t=0 . \dagger$ This function enables one to calculate the average moments

$$
\left\langle\mathbf{r}^{k}(t)\right\rangle=\int \mathbf{r}^{k} P(\mathbf{r}, t) \mathrm{d}^{d} \mathbf{r} .
$$

The probability of return to the origin (discussed in the previous section) is simply $P(0, t)$ and its value provides us with the value of $d_{\mathrm{s}}$ for the fractal medium (equation $(2.8 b))$. In addition, $P(\mathbf{r}, t)$ plays an important role in the Flory theory for SAWs [10] (see section 8.4 for details).

For a diffusion problem in Euclidean space [14],

$$
P(\mathbf{r}, t)=\left(\frac{d}{2 \pi a^{2} t}\right)^{d / 2} \exp \left(-\frac{d r^{2}}{2 a^{2} t}\right),
$$

where $a^{2}$ is the mean-square distance travelled in unit time. We show now that this simple Gaussian behaviour does not generalize to disordered (fractal) substrates. As an example, consider the relatively simple case of diffusion on self-avoiding walks. SAWs have a random fractal structure [24, 68], but are topologically linear. Therefore the diffusion process along a SAW is normal, just as in one-dimensional space. Thus, using the number of SAW steps, $l$, as a (chemical) distance coordinate [57, 109] rather than the Euclidean spatial distance $r$, one has, for $\phi(l, t)$, the probability density to find the walker at a distance $l$ at time $t$, 


$$
\phi(l, t)=\frac{1}{(2 \pi t)^{1 / 2}} \exp \left(-\frac{l^{2}}{2 t}\right) .
$$

Here we have assumed that each SAW step is of unit length. The relationship between the number of steps of a SAW and the spatial coordinate is given by the fractal dimensionality of the SAW, $r(l)^{d_{\mathrm{f}}} \sim l$. However, more detailed information is obtained from the probability density [110] $P(r, l)$, that an $l$-step SAW section is of length $r$ :

$$
P(r, l)=A_{l}\left(\frac{r}{l^{\nu}}\right)^{g} \exp \left[-a\left(\frac{r}{l^{\nu}}\right)^{\delta}\right], \quad \delta=(1-\nu)^{-1} .
$$

Here $g=(\gamma-1) / \nu$, and $\nu=1 / d_{\mathrm{f}}$ is the end-to-end exponent for the SAW. We combine (2.15) and (2.16) in order to get the probability density $P_{\mathrm{SAW}}(r, t)$ that a random walker on a SAW is a distance $r$ at time $t$ after starting from the origin at $t=0$ :

$$
P_{\mathrm{SAW}}(r, t)=\int_{0}^{\infty} P(r, l) \phi(l, t) \mathrm{d} l .
$$

Using a steepest-descent approximation, and the fact that for diffusion on a SAW $d_{\mathrm{w}}=2 d_{\mathrm{f}}$, we find for the exponential term

$$
P_{\mathrm{SAW}}(\mathbf{r}, t) \sim \exp \left[-\left(\frac{r}{t^{1 / d_{\mathrm{w}}}}\right)^{u}\right], \quad u=\left(1-\frac{1}{d_{\mathrm{w}}}\right)^{-1} .
$$

For $\nu=1, d_{\mathrm{w}}=2$ (normal one-dimensional space) we recover the Gaussian distribution (2.14), but for $\nu<1$ (as is the case for SAWs in $d>1$ ) $P_{\mathrm{SAW}}(\mathbf{r}, t)$ has a nonGaussian structure. Note, however, the striking similarity between $P_{\mathrm{SAW}}(\mathbf{r}, t)$ of (2.18) and $P(r, l)$ of (2.16). This similarity becomes evident when one realizes that $1 / d_{\mathrm{w}}$ is in fact $\nu_{\mathrm{w}}$, the end-to-end exponent for the path of the random walker. We see that the convoluted fractal structure of the SAW gives rise to a non-Gaussian probability density. We expect to find this non-Gaussian behaviour in more complex fractal media as well (see section 4.4).

A recent topic of interest is the scaling behaviour (if any) of probability densities [111-114]. The simplest scaling form that one expects is

$$
P(\mathbf{r}, t)=\frac{r^{d_{\mathrm{f}}-d}}{t^{d_{\mathrm{s}} / 2}} \Pi\left(\frac{r}{t^{1 / d_{\mathrm{w}}}}\right) .
$$

Here the scaling of $r$ with $t^{1 / d_{\mathrm{w}}}$ comes from the anomalous-diffusion theory, and the normalizing factor of $t^{d_{\mathrm{s}} / 2}$ is required because of (2.9). The $r^{d_{\mathrm{f}}-d}$ factor accounts for the scaling of the density of the fractal substrate. Note that the probability density for diffusion on a SAW, (2.18), scales as suggested in (2.19).

Several authors $[111,112]$ have considered the scaling form of $P(\mathbf{r}, t)$ on the Sierpinski gasket, exploiting the Markov property of a random walker, and using some approximations, to derive

$$
P(\mathbf{r}, t)=\frac{r^{d_{\mathrm{f}}-d}}{t^{d_{\mathrm{s}} / 2}} \exp \left(-\frac{r^{d_{\mathrm{w}}}}{t}\right) .
$$

It was suggested [111] that (2.20) is valid for all fractals. This is in disagreement with (2.18) and with the result presented by Guyer [113], who used a numerical renormalization, to obtain for the Sierpinski gasket 


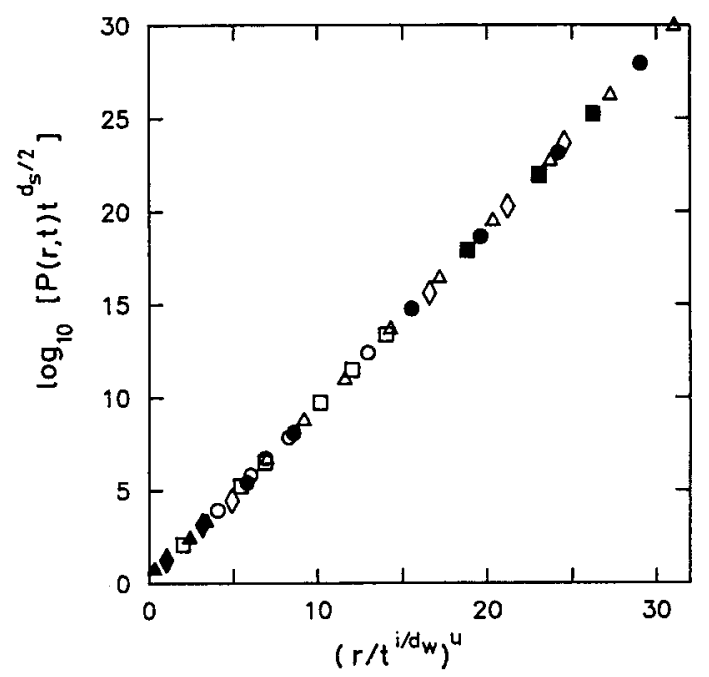

Figure 9. Plot of $\log _{10}\left[P(r, t) t^{d_{\mathrm{s}} / 2}\right]$ against $\left(r / t^{1 / d_{\mathrm{w}}}\right)^{u}$ for a Sierpinski gasket. The best linear fit is achieved for $u=1.9 \pm 0.1$.

$$
P(\mathbf{r}, t)=\frac{r^{d_{\mathrm{f}}-d}}{t^{d_{\mathrm{s}} / 2}} \exp \left[-\left(\frac{r}{t^{1 / d_{\mathrm{w}}}}\right)^{\mu}\right], \quad u=\left(1-\frac{1}{d_{\mathrm{w}}}\right)^{-1}=1.76 .
$$

Note the similarity between this result and the probability distribution of a RW on a SAW, (2.18). This form of probability distribution is found also for some loopless structures (other than SAWs) (see section 4). S. Havlin and J. Kiefer (unpublished work) find yet a different value for the exponent $u$, for random walks on the Sierpinski gasket, using the exact enumeration technique (section A.2 of the appendix), $u=1.9 \pm 0.1$. Numerical results (figure 9) show good agreement with the scaling form of (2.19). A best fit to (2.21) yields $u=1.9 \pm 0.1$, which is close to but somewhat different from the value of $u$ obtained by Guyer. It is possible [113] that the differences between (2.20) and (2.21) are due to the different ranges of validity of $P(r, t)$ in the two equations. The theoretical argument of reference [111] exploits the Markov property, and the range $r<t^{1 / d_{\mathrm{w}}}$ is of great importance. However, the renormalization procedure of Guyer holds for $r>t^{1 / d_{\mathrm{w}}}$. It was suggested by Guyer [113] that (2.20) might better describe the regime $r<t^{1 / d_{\mathrm{w}}}$, while (2.21) describes the case for which $r>t^{1 / d_{\mathrm{w}}}$. The question of the exact form of $P(\mathbf{r}, t)$ for the Sierpinski gasket is not yet resolved, and more decisive research work is necessary. Similar remarks apply to $P(\mathbf{r}, t)$ on percolation clusters (see reference [114]). We present further details on this particular problem in section 3.5.

A direct consequence of the scaling of $P(\mathbf{r}, t),(2.19)$, is the existence of a gap exponent charcterizing all the moments of diffusion. Indeed, (2.19) yields

$$
\left\langle\mathbf{r}^{k}\right\rangle=\int P(\mathbf{r}, t) \mathbf{r}^{k} \mathrm{~d}^{d} \mathbf{r}=\int \frac{r^{d_{\mathrm{f}}-d}}{t^{d_{\mathrm{s}} / 2}} \Pi\left(\frac{r}{t^{1 / d_{\mathrm{w}}}}\right) \mathbf{r}^{k} \mathrm{~d}^{d} \mathbf{r}=\text { const } \times t^{k / d_{\mathrm{w}}} .
$$

Thus $1 / d_{\mathrm{w}}$ is the gap exponent.

Recently, there have been many studies of cases of fractals, and the physical phenomena associated with fractal structures, that are not characterized by a single gap exponent, but rather by an infinite hierarchy of exponents [115-123]. The lack of 
a gap exponent rules out a scaling form like (2.19), and one of the questions to be asked is whether this is the case for diffusion or fractals. If so, this might be the reason for the many different results for $P(\mathbf{r}, t)$ presented above. For further details on this subject see section 3.5.

Another probability distribution of interest is $S(\mathbf{r}, t ; R)$, the survival probability for a diffusing particle starting at the origin at $t=0$, and surrounded by a perfectly absorbing sphere of radius $R$. This function has proved to be essential for the understanding of the physics of trapping [126-131]. In Euclidean space $S(0, t ; R) \sim \exp \left(-\gamma_{d} t / R^{2}\right)$, where $\gamma_{d}$ is a dimension-dependent constant [127]. In the case of a random medium, we expect

$$
S(0, t ; R) \sim \exp \left(-c t / R^{d_{\mathrm{w}}}\right) .
$$

This simple theoretical prediction is supported by various numerical test (B. L. Trus, S. Havlin and D. Stauffer, preprint, 1987). We shall return to this probability density and its applications in section 7.1.

Several other physical properties have been studied on the Sierpinski gasket. The elasticity of the Sierpinski gasket has been studied by Bergman and Kantor [132], trapping on the Sierpinski gasket by Zumofen et al. [133], rate processes by Kopelman [41, 135] and waiting-time distribution by Robillard and Tremblay [136]. The solution and the spectrum of the Schrödinger equation on the Sierpinski gasket has been studied by Domany et al. [137] and by Rammal and Toulouse [138].

\section{Diffusion on percolation clusters}

The percolation model [139, 140] has been found useful to characterize many disordered systems, such as porous media, fragmentation and fractures [2], gelation $[8,10]$, random-resistor insulator systems [20, 141], dispersed ionic conductors [142], forest fires [18, 143] and epidemics [144, 145]. For simplicity, we describe the site percolation model [140] on a square lattice. Consider a simple square lattice for which a fraction $p$ of sites are randomly occupied and a fraction $1-p$ are empty. Nearest-neighbour occupied sites form connected clusters. When $p$ increases, the average size of the clusters increases. There exists [146] a critical concentration $p_{\mathrm{c}}=0.592745$ below which only finite clusters exist and above which a large ('infinite') cluster is formed (see figure 10). The percolation transition at $p_{\mathrm{c}}$ is described by the probability $P_{\infty}$ that a site in the lattice belongs to the incipient infinite cluster. Below $p_{\mathrm{c}}, P_{\infty}=0$, whereas above $p_{\mathrm{c}}, P_{\infty}$ increases with $p$ as

$$
P_{\infty} \sim\left(p-p_{\mathrm{c}}\right)^{\beta} \text {. }
$$

The diameter of the clusters below $p_{\mathrm{c}}$ is characterized by the correlation length $\xi$ which can be regarded as the mean distance between two sites belonging to the same cluster. When $p \rightarrow p_{\mathrm{c}}, \xi$ diverges as

$$
\xi \sim\left(p_{\mathrm{c}}-p\right)^{-\nu}
$$

The exponents $\beta$ and $\nu$ are universal and depend only on the space dimensionality $d$ but not on the lattice structure. For $d=2$ the critical exponents are known exactly [147] to be $\beta=\frac{5}{36}$ and $\nu=\frac{4}{3}$. For $d=3$ there exist numerical estimates [148, 149], $\beta \approx 0.44$ and $\nu \approx 0.88$. The upper critical dimension above which the mean-field theory $[125,161]$ is valid is $d=d_{\mathrm{c}}=6$. Mean-field percolation can be modelled by 


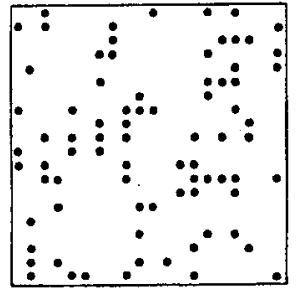

$p=0.2$

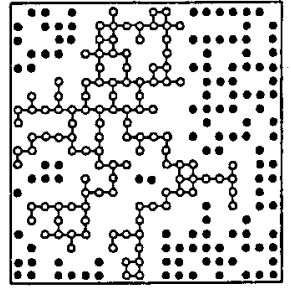

$p=0.59$

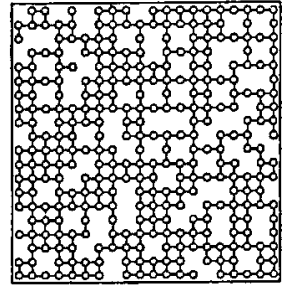

$p=0.8$

Figure 10. Square lattice of $20 \times 20$. Sites have been randomly occupied with probability $p$ $(p=0.20,0.59,0.80)$. Sites belonging to finite clusters are marked by full circles, and sites on the 'infinite' clusters are marked by open circles. (After Bunde [13].)

percolation on a Cayley tree (Bethe lattice). This model has been solved exactly [150], yielding $\beta=1$ and $\nu=\frac{1}{2}$. For recent reviews on percolation see references [16-22].

The problem of diffusion on percolation clusters provides us with one of the most valuable tools for the study of the topological properties of the clusters. Different geometrical models, such as the de Gennes model [106] and the links-and-nodes model of Skal and Shklovski [107] for percolation, lead to different predictions for diffusion. The model that is most favoured is the fractal model introduced by Kirkpatrick [16] and further developed by Gefen et al. [44], Mandelbrot and Given [151] and de Arcangelis et al. [116]. Other geometrical models, such as the bubble model (S. Redner and B. Kahng, private communication) for percolation, are currently being studied. The Alexander-Orbach conjecture [45] that the fracton dimensionality of percolation clusters at criticality is $\frac{4}{3}$ for any Euclidean dimension $d>1$ has attracted considerable interest.

In this section we review the analogy of percolation to exact fractal lattices, the theoretical and numerical results $[47,81-83,152]$ for diffusion on percolation, and their relationship to static exponents-describing the structure of the clusters. We also review the chemical distance and its application to transport, and recent results for continuum percolation [153-163].

\subsection{Analogy with diffusion on fractals}

A great amount of research [44, 151, 164] has led to the conclusion that percolation clusters are fairly well described by fractals. The picture is as follows. For $p<p_{\mathrm{c}}$ (below the percolation threshold) there appear clusters of finite size with a typical linear size $\xi(p)$. In fact $\xi(p)$ can be regarded as the mean size of the clusters or as their maximal size. There also appear clusters of a size much larger than $\xi$. These are lattice animals $[141,165]$, and we neglect them in the present discussion because of their scarcity (see, however, section 4.2). The clusters generated at $p<p_{\mathrm{c}}$ each have a statistical internal self-similarity property, which is characteristic of fractals. This has been demonstrated by Sapoval et al. [166]. Actually, the fractal dimensionality of these clusters has been shown to be $d_{\mathrm{f}}=d-\beta / \nu$, both theoretically $[16,18,167,168]$ and numerically [164]. Thus, $d_{\mathrm{f}}=\frac{91}{48} \approx 1.896$ in $d=2$, $d_{\mathrm{f}} \approx 2.5$ in $d=3$, and $d_{\mathrm{f}}=4$ in $d=d_{\mathrm{c}}=6$. Percolation clusters at this regime are therefore successfully modelled by exact fractal lattices of linear size $\xi(p)$, with the corresponding spatial properties of fractal and fracton dimensionality, connectivity, dead ends, etc. $[44,116,151]$. As $p$ approaches $p_{\mathrm{c}}$ from below, the characteristic size of the finite clusters increases, until at $p=p_{\mathrm{c}}$ there appears an infinite self-similar 


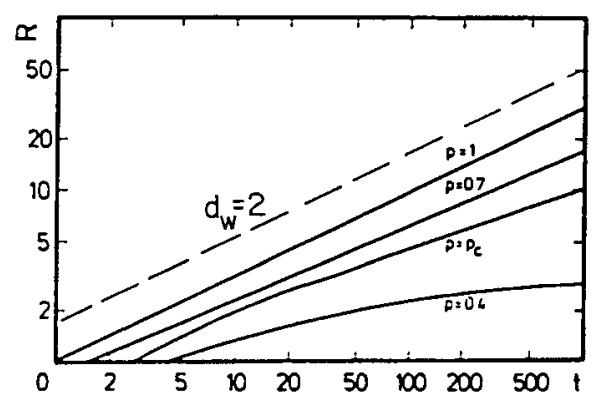

Figure 11. Example of $R \equiv\left\langle R^{2}(t)\right\rangle^{1 / 2}$ for diffusion on percolation clusters generated at $p>p_{\mathrm{c}}, p=p_{\mathrm{c}}$ and $p<p_{\mathrm{c}}$ on a square lattice. The dashed line with slope $\frac{1}{2}$, representing $d_{\mathrm{w}}=2$, is shown for comparison. (After Stauffer [18].)

cluster (with similar spatial characteristics to those of the finite clusters for $p<p_{\mathrm{c}}$ ). The incipient infinite cluster is then modelled by an infinite fractal lattice, similar to the lattices modelling the clusters for $p<p_{\mathrm{c}}$. For $p \geqslant p_{\mathrm{c}}$ there are also finite clusters in the empty spaces left by the incipient infinite cluster. These are quite similar to the finite clusters below $p_{\mathrm{c}}$. Above the percolation threshold $\left(p>p_{\mathrm{c}}\right)$ there is an infinite cluster, but a finite correlation length $\xi(p)$. We interpret $\xi(p)$ as being a typical distance up to which the infinite cluster is statistically self-similar. As an example [164], the mass of the infinite cluster scale as $M \sim R^{d_{\mathrm{f}}}$ for $R<\xi(p)$, but for $R>\xi(p)$ self-similarity of the fractal is lost and the mass scales on the average as for a homogeneous object, $M \sim R^{d}$. Thus the infinite cluster for $p>p_{\mathrm{c}}$ might be modelled by a regular lattice of fractal unit cels. Each fractal unit cell is of length $\xi(p)$ and similar in structure to the fractal lattices modelling percolation for $p \leqslant p_{\mathrm{c}}$.

There are three characteristic regimes for diffusion on percolation clusters. For $p>p_{\mathrm{c}}$ the infinite cluster is homogeneous for $R>\xi(p)$, and diffusion is regular, with $d_{\mathrm{w}}=2$. At criticality, $p=p_{\mathrm{c}}$, the incipient infinite cluster is self-similar in all length scales, and anomalous diffusion takes place, with $d_{\mathrm{w}}>2$. Finally, for $p<p_{\mathrm{c}}$, the largest clusters are typically of a finite linear size $\xi(p)$, and $\left\langle R(t)^{2}\right\rangle \sim \xi^{2}(p)$ when $t \rightarrow \infty$. These three regimes are illustrated in figure 11 .

\subsection{Two ensembles}

When studying properties of percolation clusters, it is convenient to look at one of two possible ensembles. The first kind of statistical ensemble includes only percolation clusters whose linear size is much larger than the typical length of the physical phenomenon under discussion. Thus, since we are interested in diffusion, such an ensemble will include those clusters whose size is much larger than the mean span of diffusion. This ensemble, of large clusters, is usually generated at $p$ slightly below $p_{\mathrm{c}}$, and therefore its clusters have the same self-similarity as the incipient infinite cluster. Since the phenomenon being studied is of a linear size much smaller than that of the cluster, end-effects are completely avoided, and for all practical purposes the results are equivalent to those that one would have obtained from the incipient infinite cluster. The importance of this ensemble lies in the fact that it gives critical exponents equal in value to those usually defined for the percolation transition (critical exponents are traditionally defined for the properties of the incipient infinite cluster alone). 
The other statistical ensemble is one that includes all the percolation clusters (again usually for $p<p_{\mathrm{c}}$ ). This is independent of the typical linear size of the phenomenon studied. Thus, for example, such an ensemble will include clusters whose size is smaller than the typical diffusion span (as well as all clusters with a larger size). This ensemble is natural, since this is the unrestricted ensemble that one obtains in a simple percolation experiment. We denote the critical exponents measured on this ensemble by a prime.

We now present $[47,48]$ some simple arguments for the relation between $d_{\mathrm{w}}^{\prime}$, the anomalous diffusion exponent for an ant 'dropped' on the ensemble of all percolation clusters, and $d_{\mathrm{w}}$, the corresponding diffusion exponent on the incipient infinite cluster at $p=p_{\mathrm{c}}$. For the ensemble of all clusters, diffusion can start on any of the clusters, and consequently there exist two regimes. One is for clusters whose linear size is smaller than the mean span of diffusion, and the other is for clusters for which the random walker never reaches its edges (the clusters being too large). Then, roughly, for a $t$-step random walk performed on an $S$-cluster (i.e. a cluster consisting of $S$ sites), we expect

$$
r_{\mathrm{s}}^{2}(t) \sim \begin{cases}t^{2 / d_{\mathrm{w}}} & \left(t^{2 / d_{\mathrm{w}}}<R_{\mathrm{s}}^{2}\right) \\ R_{\mathrm{s}}^{2} & \left(t^{2 / d_{\mathrm{w}}}>R_{\mathrm{s}}^{2}\right),\end{cases}
$$

where $R_{\mathrm{s}}^{2}$ is the average squared size of an $S$-cluster:

$$
R_{\mathrm{s}}^{2} \sim S^{2 / d_{\mathrm{f}}}
$$

The mean $\left\langle r^{2}(t)\right\rangle$ on all clusters can be calculated taking into account the probability $S^{1-\tau}$ that the ant starts diffusing on an $S$-cluster (at $p=p_{\mathrm{c}}$ )

$$
\begin{aligned}
\left\langle r^{2}(t)\right\rangle_{p=p_{\mathrm{c}}} & \sim \sum_{S=1}^{\infty} S^{1-\tau} r_{\mathrm{s}}^{2}(t) \sim \sum_{S=1}^{S_{\text {cross }}} S^{1-\tau} S^{2 / d_{\mathrm{f}}}+\sum_{S_{\text {cross }}}^{\infty} S^{1-\tau} t^{2 / d_{\mathrm{w}}} \\
& \sim S_{\text {cross }}^{2-\tau+2 / d_{\mathrm{f}}}+S_{\text {cross }}^{2-\tau} t^{2 / d_{\mathrm{w}}} .
\end{aligned}
$$

The quantity $S_{\text {cross }}$ is the cluster mass for which the crossover occurs in (3.3):

$$
S_{\text {cross }}^{2 / d_{\mathrm{f}}} \sim t^{2 / d_{\mathrm{w}}} \text {. }
$$

We see that the two contributions in (3.5) have the same singularity in $t$, and

$$
\left\langle r^{2}(t)\right\rangle \sim t^{2 / d_{\mathrm{w}}^{\prime}} \sim t^{2 / d_{\mathrm{w}}+(2-\tau) d_{\mathrm{f}} / d_{\mathrm{w}}}
$$

or

$$
\frac{d_{\mathrm{w}}}{d_{\mathrm{w}}^{\prime}}=1+\frac{1}{2} d_{\mathrm{f}}(2-\tau)=1-\frac{\beta}{2 \nu} .
$$

Here we have made use of the scaling relation [18] $\tau=(2 d \nu-\beta) /(d \nu-\beta)$.

\subsection{Scaling theory}

The simple arguments given in the preceding section lead to a prediction for diffusion on percolation at criticality $\left(p=p_{\mathrm{c}}\right)$. In practice, one can make numerical simulations, or experiments, for any probability $p$ below or above $p_{\mathrm{c}}$. It is therefore desirable to develop a theory that is able to predict the exponents of interest $\left(d_{\mathrm{w}}\right.$ and $\left.d_{\mathrm{w}}^{\prime}\right)$ from results at any $p$. Such a theory is the scaling theory $[47,48]$, which will now be discussed. 
We deal first with the general ensemble which consists of all (unrestricted) clusters. In the conducting phase of percolation, $p>p_{\mathrm{c}}$, the average diffusion constant

$$
D^{\prime}(t, p) \equiv\left\langle r^{2}(t)\right\rangle^{\prime} / t
$$

of a random walk is finite, in the limit $t \rightarrow \infty$. This is because for $p>p_{\mathrm{c}}$ the infinite conducting cluster is homogeneous at scales of length larger than $\xi(p)$, and a random walk behaves on it as on Euclidean space (for $\left\langle r^{2}(t)\right\rangle \gg \xi^{2}$ ). In this limit the Einstein relation (2.4) shows that the value of the diffusion constant is proportional to the d.c. conductivity $\sigma(p)$ of the cluster (note that here the density of carriers $n$ is proportional to $r^{d}$, since the mass of the infinite cluster scales for $r>\xi$ as $r^{d}$ ). Indeed, following a suggestion by de Gennes [42], (see also Kopelman [169]) computer simulations of diffusion on percolation lattices have been used $[43,170]$ to determine the conductivity exponent $\mu=\tilde{\mu} \nu$ via

$$
D^{\prime}(t \rightarrow \infty, p) \sim \sigma(p) \sim\left(p-p_{\mathrm{c}}\right)^{\mu} \quad\left(p>p_{\mathrm{c}}\right) .
$$

However, this behaviour holds only in the neighbourhood of $p_{\mathrm{c}}$, and for very large $t$, such that the average span $r(t) \equiv\left\langle r^{2}(t)\right\rangle^{1 / 2}$ of the walks is much larger than $\xi$. On the other hand, when $r^{\prime}(t) \ll \xi$, one expects behaviour that is similar to that of anomalous diffusion at the threshold $p_{\mathrm{c}}$, where $D^{\prime}(t)$ vanishes algebraically as

$$
D^{\prime}\left(t, p_{\mathrm{c}}\right) \sim t^{\left(2-d_{\mathrm{w}}^{\prime}\right) / d_{\mathrm{w}}^{\prime}} .
$$

In practice the condition $r(t) \gg \xi$ is hardly ever achieved in simulations near $p_{\mathrm{c}}$. This limits the reliability of determining $\mu$ via (2.7), and is one more reason for the need for a method that incorporates the crossover from a finite diffusion constant above $p_{\mathrm{c}},(3.9)$, to the behaviour at criticality, (3.10).

For $p>p_{\mathrm{c}}$ and $r(t) \gg \xi$ (i.e. $t \rightarrow \infty$ ), (3.9) is expected to be valid. For $p<p_{\mathrm{c}}$ and $t \rightarrow \infty$, the dominant contribution to $r^{\prime}(t)$ comes from clusters whose linear size is equal to or bigger than $\xi \sim\left|p-p_{\mathrm{c}}\right|^{-\nu}$. In each of these clusters $r^{2}(t) \sim \xi^{2}$, but the probability of being on one of these clusters is, by the usual percolation scaling assumptions, proportional to $\left|p-p_{\mathrm{c}}\right|^{\beta}$. Thus the average diffusion constant behaves below $p_{\mathrm{c}}$ as

$$
D^{\prime}(t \rightarrow \infty, p) \sim t^{-1}\left(p_{\mathrm{c}}-p\right)^{-2 \nu+\beta},
$$

as also noted by Stauffer [141]. We now combine (3.9)-(3.11) in the following scaling form:

$$
D^{\prime}(t, p)=t^{2 / d_{w}^{\prime}-1} \phi\left[\left(\frac{p}{p_{\mathrm{c}}}-1\right) t^{\left(d_{\mathrm{w}}^{\prime}-2\right) / \mu d_{w}^{\prime}}\right] .
$$

This is in agreement with the discussion behaviour of $D^{\prime}$ if

$$
\phi(x) \sim \begin{cases}x^{\mu} & \text { as } x \rightarrow \infty, \\ (-x)^{-2 \nu+\beta} & \text { as } x \rightarrow-\infty, \\ \text { const. } & \text { as } x \rightarrow 0 .\end{cases}
$$

Consistency with (3.11) in powers of $t$ requires

$$
d_{\mathrm{w}}^{\prime}=\frac{2+\mu / \nu-\beta / \nu}{1-\beta / 2 \nu} .
$$


This result was derived also by Webman [46]. The crossover from the behaviour below $p_{\mathrm{c}},(3.11)$, to that above $p_{\mathrm{c}},(3.9)$, occurs at $x_{\text {cross }}$, a fixed agrument of $\phi(x)$. Then

$$
t_{\text {cross }}^{\prime} \sim\left|p-p_{\mathrm{c}}\right|^{-\beta d_{\mathrm{w}}^{\prime} /\left(d_{\mathrm{w}}^{\prime}-2\right)}=\left|p-p_{\mathrm{c}}\right|^{\beta-2 \nu-\mu} .
$$

Very similar arguments can be made for the restricted ensemble of large clusters. Since the probability of being on clusters with sizes that are equal to or greater than $\xi$ behaves as $\left|p-p_{\mathrm{c}}\right|^{\beta}$, both above and below $p_{\mathrm{c}}$, it is expected that

(a) for $p>p_{\mathrm{c}}$ and $r(t) \gg \xi, D \sim\left(p-p_{\mathrm{c}}\right)^{\mu-\beta}$;

(b) for $p<p_{\mathrm{c}}$ and $t \rightarrow \infty, D \sim t^{-1}\left|p-p_{\mathrm{c}}\right|^{-2 \nu}$; and

(c) for $r(t) \ll \xi, D \sim t^{2 / d_{w}-1}$.

The scaling function of $D$, which is consistent with these properties, is given by

$$
D(t, p)=t^{2 / d_{\mathrm{w}}-1} g\left[\left(\frac{p}{p_{\mathrm{c}}}-1\right) t^{\left(d_{\mathrm{w}}-2\right) /(\mu-\beta) d_{\mathrm{w}}}\right],
$$

where

$$
g(x) \sim \begin{cases}x^{\mu-\beta} & \text { as } x \rightarrow \infty \\ (-x)^{-2 \nu} & \text { as } x \rightarrow-\infty \\ \text { const. } & \text { as } x \rightarrow 0\end{cases}
$$

Again consistency yields a relation between $d_{\mathrm{w}}$ and $\mu$ :

$$
d_{\mathrm{w}}=2+\frac{\mu-\beta}{\nu} \text {. }
$$

Note that this result is in agreement with (2.7), which was derived in a very different way. Also the ratio of (3.18) and (3.14) yields $d_{\mathrm{w}} / d_{\mathrm{w}}^{\prime}=1-\beta / 2 \nu$, exactly as given in (3.7). Relations between the moments of typical diffusion times and the resistive correlations in percolation systems were found recently by A. B. Harris, Y. Meir and A. Aharony (preprint, 1987).

One further interesting result is the fact that in (3.16) the crossover occurs at

$$
t_{\text {cross }} \sim\left(p-p_{\mathrm{c}}\right)^{-(\mu-\beta) d_{\mathrm{w}} /\left(d_{\mathrm{w}}-2\right)}=\left(p-p_{\mathrm{c}}\right)^{\beta-2 \nu-\mu},
$$

which scales exactly the same as $t_{\text {cross }}^{\prime}$. This crossover, derived also by Straley [134], is also reflected in a crossover in the density of scales $\mathscr{N}(\epsilon)$ in the corresponding vibrational problem [171-173]. For percolation systems above $p_{\mathrm{c}}, \mathscr{N}(\epsilon)$ crosses over from a phonon behaviour $\epsilon^{d / 2-1}$ for small $\epsilon$ (large $t$ ) to a fracton behaviour $\mathscr{N}(\epsilon) \sim \epsilon^{d_{\mathrm{s}} / 2-1}$ for large $\epsilon$. Such a crossover has been observed experimentally in randomly dilute magnetic systems [31, 32], in silica aerogels [33], in silica smokeparticle aggregates (D. Richter, T. Freltoft and J. K. Kjems, preprint, 1987) and in glassy ionic conductors [34].

\subsection{Chemical distance metric}

The chemical distance between two sites is defined as the shortest path on the structure connecting the two sites. This concept has recently been studied very extensively [57, 174-176, 177-180, 186-188], and has been found [52, 180, 181, 182] to be useful in characterizing the growth of random aggregates [84, 182], the spreading of forest fires [181] or epidemics, and in particular the transport properties of loopless aggregates [52, 182]. For loopless aggregates, the chemical distance 


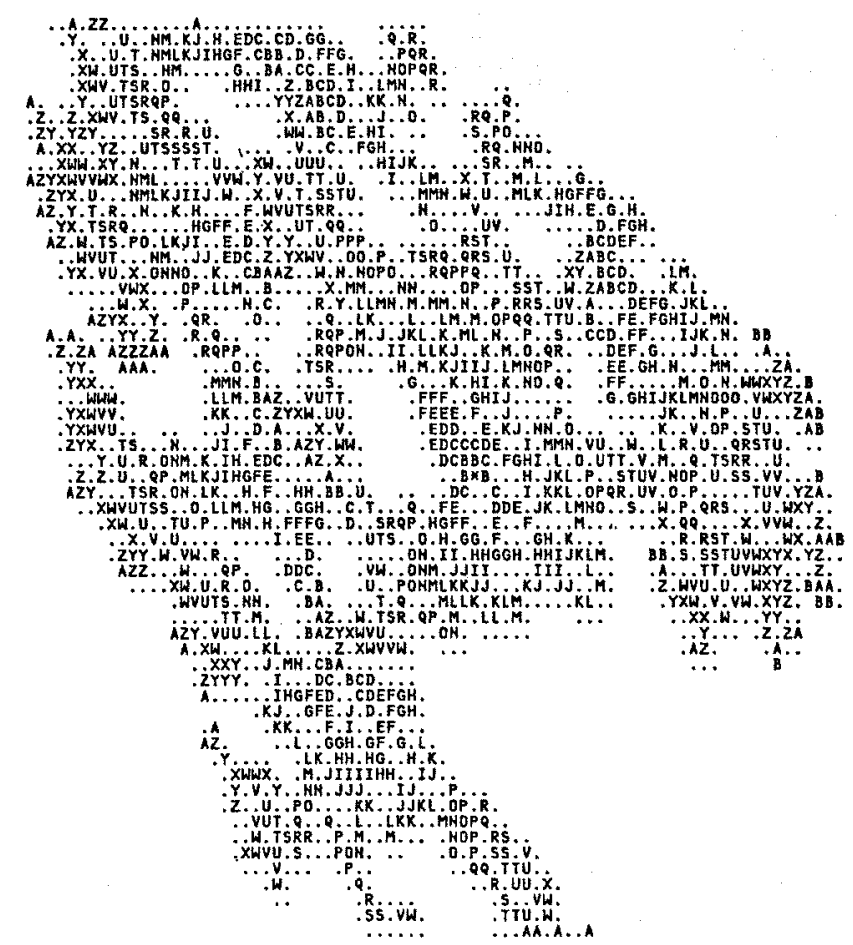

Figure 12. Example of a part of an infinite percolation cluster grown at criticality. The asterisk $*$ is an arbitrary point in the cluster. Sites belonging to shells of successive chemical distance are designated by successive letters of the alphabet (starting with B), and dots designate sites that were tested and found to be empty. The cluster was generated on a triangular lattice, obtained by a square lattice regarding sites along one of the diagonal directions as nearest neighbours.

properties determine entirely the dynamics of the system (see section 4). Using the concept of chemical distance, one can obtain bounds [183] on the transport critical exponents for disordered aggregates such as percolation clusters. This is achieved by neglecting the effect of loops in these structures.

In the following we introduce one new exponent related to the chemical distance. In figure 12 we show [57] a part of the infinite percolation cluster at criticality generated on a triangular lattice using the growth method of Alexandrowicz [175] and Leath [184]. Occupied sites of the cluster are designated by letters. Dots represent unoccupied perimeter sites and blank spaces represent non-perimeter unoccupied sites. Suppose that one chooses an arbitrary reference point and designates it by an asterisk. The occupied nearest neighbours of that site, designated by $\mathrm{B}$, represent a 'shell' lying at chemical distance $l=1$ from the reference site. The next-nearest-neighbou $\mathrm{r}$ sites are designated by $\mathrm{C}$ and constitute a shell at chemical distance $l=2$, and so forth. Thus $l$ is the smallest number of nearest-neighbour connections within the cluster, by which a site is separated from the origin. We define the following quantities, which are functions of $l: G(l)$ is the number of occupied sites in the $l$ th shell, $S(l)$ is the total number of occupied sites up to the $l$ th shell, and $R(l)$ is the average Pythagorean distance between the reference site and a site in the $l$ th shell. 
The average quantities $\bar{G}(l), \bar{S}(l)$ and $\bar{R}(l)$ for the incipient infinite percolation cluster at $p_{\mathrm{c}}$ can be related as follows. We assume that these averages vary as powers of $l$. The chemical-distance exponent $\tilde{\nu}$ is defined [57] by

$$
\overline{R^{2}} \sim l^{2 \tilde{\nu}} \equiv l^{2 / d_{\min }}
$$

The exponent $d_{\min }$ can be understood as the fractal dimension of the shortest path [185], i.e. the path of minimum length. For percolation [57] in $d=2, \tilde{\nu}=0.88$ and for $d=3, \tilde{\nu} \approx 0.75$. For $d=d_{\mathrm{c}}=6$ the mean-field value is exactly $\tilde{\nu}=\frac{1}{2}$. For mean field, percolation interactions and long-range correlations can be neglected and the chemical paths behave like random walks with $\tilde{\nu}=\frac{1}{2}$. A value of $\tilde{\nu}$ less than 1 indicates that the chemical paths are winding and have a fractal nature similar to that of the Koch curve [24] or of self-avoiding walks. Most numerical simulations $[57,175-179,180,186]$ give $\tilde{\nu} \approx 0.88<1$. This is in disagreement with the numerical results of Edwards and Kerstein [187], who find that $\nu \approx 1$ for two-dimensional percolation clusters. Since $S \sim R^{d_{\mathrm{f}}}$ and $\mathrm{d} \bar{S} / \mathrm{d} l=\bar{G}$, it follows that

and

$$
\bar{S}(l) \sim l^{\tilde{\nu} d_{\mathrm{f}}} \equiv l^{\mathrm{d}_{l}}
$$

$$
\bar{G}(l) \sim l^{\tilde{\nu} d_{\mathrm{f}}-1} \sim l^{d_{l}-1} .
$$

The exponent $d_{l}$ is the chemical-space analogue of the fractal dimension exponent $d_{\mathrm{f}}$. Note the $d_{l}$ is not a new exponent, but is related to $\tilde{\nu}$ by $d_{l}=\tilde{\nu} d_{\mathrm{f}}$.

In the Pythagorean $R$-metric $S(R)$ scales as [141]

$$
\bar{S}(R) \sim R^{d} P_{\infty} f(R / \xi) \sim R^{d} \xi^{-\beta / \nu} f(x),
$$

where $x \equiv R / \xi$, and

$$
f(x) \sim \begin{cases}\text { const } & (x \gg 1), \\ x^{-\beta / \nu} & (x \ll 1) .\end{cases}
$$

From $f(x \ll 1) \sim x^{-\beta / \nu}$, it follows, using (3.23), that $\bar{S}(R) \sim R^{d-\beta / \nu}$ (for $\left.R \ll \xi\right)$, thus recovering $d_{\mathrm{f}}=d-\beta / \nu$.

In analogy with the above, a scaling relation can be written for the $l$-metric:

$$
\bar{S}(l) \sim l^{\tilde{\nu} d} \xi_{l}^{-\beta \tilde{\nu} / \nu} g\left(l / \xi_{l}\right)
$$

Here $\xi_{l}$ is the chemical correlation length [57], i.e. the correlation length as measured in the chemical metric $\left(\xi_{l} \geqslant \xi\right)$. Since for $y \equiv l / \xi_{l} \gg 1, \bar{S} \sim l^{d}$, and for $y \ll 1, \bar{S}$ is independent of $\xi_{l}$, we have

$$
g(y) \sim \begin{cases}y^{-\tilde{\nu} d+d} & (y \gg 1), \\ y^{-\beta \tilde{\nu} / \nu} & (y \ll 1) .\end{cases}
$$

This is in accordance with (3.21), that $d_{l}=\tilde{\nu}(d-\beta / \nu)=\tilde{\nu} d_{\mathrm{f}}$.

The conditional probability density $P(r \mid l)$ for the geometric distance $r$ corresponding to a given chemical distance $l$ has been studied recently [109]. In analogy with the theory of SAWs [10, 110], it was suggested [109] that

$$
P(r \mid l)=A_{l}\left(\frac{r}{l^{\tilde{\nu}}}\right)^{\tilde{g}} \exp \left[-a\left(\frac{r}{l^{\tilde{\nu}}}\right)^{\tilde{\delta}}\right],
$$

and that $\tilde{\delta}=(1-\tilde{\nu})^{-1}$. Numerical simulations for percolation clusters in $d=2$ are in very good agreement with (3.27), with $\tilde{g}=2.5 \pm 0.3, \tilde{\nu}=0.88 \pm 0.02$ (see figure 


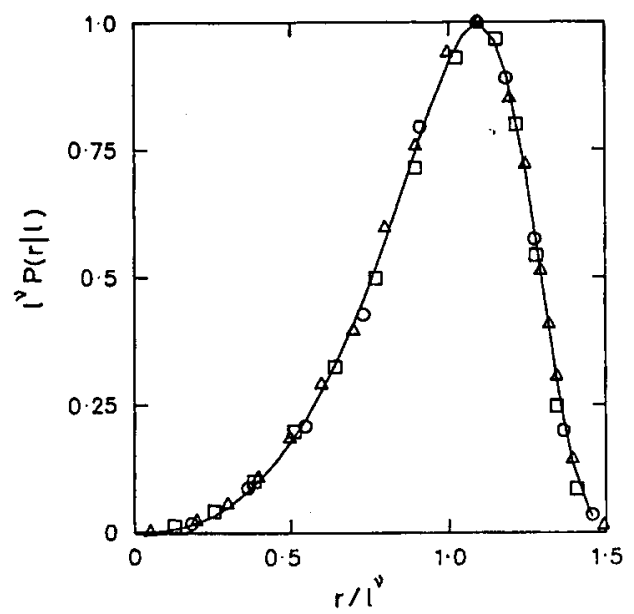

Figure 13. The conditional probability density $P(r \mid l)$ for the geometric distance $r$ corresponding to a given chemical distance $l$. Results for $l=50(\diamond), 100(\bigcirc)$, $150(\square)$ and $200(\triangle)$ are shown. The solid line represents the best fit of (3.27) to the data.

13). The distribution $P(r \mid l)$ is useful for studying the probability distributions of random walks on fractals, as, for example, in the derivation of $P(r, t)$ of random walks on SAWs in section 2.4. $P(r, t)$ for percolation clusters will be discussed in the next section.

Dynamical properties such as conductivity and diffusion can also be expressed in terms of the chemical distance. The chemical-diffusion exponent $d_{\mathrm{w}}^{l}$ characterizes the average chemical distance traversed in $t$ steps by a random walk on the infinite cluster [57]:

$$
l^{d_{\mathrm{w}}^{l}} \sim t .
$$

Using $R \sim l^{\tilde{\nu}}$, it follows that

$$
d_{\mathrm{w}}^{l}=\tilde{\nu} d_{\mathrm{w}} .
$$

Similarly, the fracton dimension can be expressed as

$$
d_{\mathrm{s}}=\frac{2 d_{l}}{d_{\mathrm{w}}^{l}}=\frac{2 d_{\mathrm{f}}}{d_{\mathrm{w}}} .
$$

For the case of loopless aggregates or aggregates for which loops can be neglected, it can be shown (for details see [52] and section 4.1) that

$$
d_{\mathrm{w}}=d_{\mathrm{f}}\left(1+\frac{1}{d_{l}}\right), \quad d_{\mathrm{w}}^{l}=d_{l}+1, \quad d_{\mathrm{s}}=\frac{2 d_{l}}{d_{l}+1} .
$$

Thus, in this case, the dynamics is entirely controlled by $d_{l}$ and $d_{\mathrm{f}}$. In particular, these results are valid for percolation clusters in $d \geqslant 6$, for which loops can be neglected, in this case, $d_{l}=2$, and hence $d_{\mathrm{w}}^{l}=3$ and $d_{\mathrm{s}}=\frac{4}{3}$, as expected [45].

The dynamical exponents for the percolation system in $d<6$ cannot be calculated using (3.31), since loops are relevant for all length scales. However, one can use (3.31) to obtain [183] bounds for percolation. In this case, the resistance exponent is bounded by 
Table 1. Bounds for the transport exponents of percolation clusters from (3.32)-(3.34). The best known values of the actual exponents are given in parentheses.

\begin{tabular}{|c|c|c|c|c|c|c|c|c|c|}
\hline \multirow[b]{2}{*}{$d$} & \multicolumn{3}{|c|}{$\tilde{\zeta}$} & \multicolumn{3}{|c|}{$d_{\mathrm{w}}$} & \multicolumn{3}{|c|}{$d_{\mathrm{s}}$} \\
\hline & $\begin{array}{l}\text { Lower } \\
\text { bound }\end{array}$ & & $\begin{array}{l}\text { Upper } \\
\text { bound }\end{array}$ & $\begin{array}{l}\text { Lower } \\
\text { bound }\end{array}$ & & $\begin{array}{l}\text { Upper } \\
\text { bound }\end{array}$ & $\begin{array}{l}\text { Lower } \\
\text { bound }\end{array}$ & & $\begin{array}{l}\text { Upper } \\
\text { bound }\end{array}$ \\
\hline 2 & 0.75 & $(0.97)$ & 1.15 & $127 / 48$ & $(2.87)$ & 3.03 & 1.24 & (1.3) & 1.43 \\
\hline 3 & 1.14 & (1.27) & 1.36 & 3.64 & (3.68) & 3.86 & 1.30 & (1.33) & 1.37 \\
\hline 4 & 1.43 & (1.54) & 1.58 & 4.49 & & 4.64 & 1.32 & (1.33) & 1.36 \\
\hline 6 & 2 & & 2 & 6 & & 6 & $4 / 3$ & & $4 / 3$ \\
\hline
\end{tabular}

$$
\frac{1}{\nu} \leqslant \tilde{\zeta} \leqslant \frac{1}{\tilde{\nu}}
$$

The upper bound is the resistance exponent of the shortest path between sites separated by a distance $R$ (see section 4.1). The actual resistance is lower because of the effect of loops. The lower bound is the exponent for singly connected (red) bonds $[150,176]$, which have a lower resistance since we neglect the contribution of blobs. These bounds were used also in the context of elasticity [189]. Combining $(2.7 b), d_{\mathrm{w}}=d_{\mathrm{f}}+\tilde{\zeta}$ and (3.32), one obtains

$$
d_{\mathrm{f}}+\frac{1}{\nu} \leqslant d_{\mathrm{w}} \leqslant d_{\mathrm{f}}+\frac{1}{\tilde{\nu}}
$$

and

$$
\frac{2 d_{\mathrm{f}}}{d_{\mathrm{f}}+1 / \tilde{\nu}}=\frac{2 d_{l}}{d_{l}+1} \leqslant d_{\mathrm{s}} \leqslant \frac{2 d_{\mathrm{f}}}{d_{\mathrm{f}}+1 / \nu} .
$$

The two bounds in (3.32)-(3.34) become identical for percolation systems in $d \geqslant 6$, since in this case $\tilde{\nu}=\nu=\frac{1}{2}$. For percolation in $d=2-4$ the two bounds are estimated from the known best values of $\nu, \tilde{\nu}, d_{l}$ and $d_{\mathrm{f}}$ (section 3.6) and are presented in table 1 .

The question of the origin of the chemical exponents $d_{l}$ and $\tilde{\nu}$ is of great interest. Is $d_{l}$ a 'new' exponent independent of the known percolation exponents $(\beta, \nu)$ or not? A Flory-type argument $[183,190,191]$ relates $\tilde{\nu}$ to $d_{\mathrm{f}}$, but only in an approximate fashion, providing an incomplete answer to this question. The simplest Flory formula was first derived as [183]:

$$
\tilde{\nu}=\frac{4}{d+2}, \quad d \leqslant 6 .
$$

This result was also obtained by Family and Coniglio [190]. A similar Flory type formula for $\tilde{\nu}$ was suggested by Roux [191]:

$$
\tilde{\nu}=\frac{2}{d+2-d_{\mathrm{f}}}, \quad d \leqslant 6 .
$$

Using the Flory expression [192] for $d_{\mathrm{f}}=\frac{1}{2}(d+2)$, $(3.35 b)$ reduces to $(3.35 a)$. However, substituting the exact values of $d_{\mathrm{f}}$ in $(3.35 b)$ yields better agreement with the measured numerical values for $\tilde{\nu}$ in $d=2$. 
A formula relating $\tilde{\nu}$ to the known exponents $(\nu, \beta)$ of percolation was conjectured by Havlin and Nossal [57]:

$$
d_{\mathrm{f}}=\frac{1}{\nu}+\frac{1}{\tilde{\nu}}
$$

This relation was based on numerical data given at that time. Recent numerical data presented by Grassberger [186] as well as an $\epsilon$-expansion near six dimensions [194] are not consistent with (3.36). Thus the question as to whether $\tilde{\nu}$ is a new independent exponent is still open. Recent numerical estimates of $\tilde{\nu}$ and $d_{l}$ will be presented in section 3.6.

A quantity of interest related to $\tilde{\nu}$ is the velocity in which a fire front or an epidemic propagates (wetting velocity). Using percolation as a model, assume that a fire starts from an occupied percolation site and propagates by burning one chemical shell per unit time. The velocity of the fire is [186, 195]

$$
v=\frac{\mathrm{d} r}{\mathrm{~d} t} \equiv \frac{\mathrm{d} r}{\mathrm{~d} l} \sim l^{\tilde{\nu}-1} \sim\left(p-p_{\mathrm{c}}\right)^{-\nu(1-\nu / \tilde{\nu})} .
$$

Since $\nu(1-1 / \tilde{\nu}) \approx 0.16$ is very small (in $d=2$ ), it follows that the increase of $v$ upon crossing $p_{\mathrm{c}}$ is very steep. A fire that does not propagate at all at $p$ slightly below $p_{\mathrm{c}}$ will propagate very fast just above $p_{\mathrm{c}}$. This propagation process was proposed by Ritzenberg and Cohen to study the spread of electrical activity in the heart [180]. Wetting velocity near the directed percolation threshold was studied by Khantha and Yeomans [124].

\subsection{Probability densities and multifractals}

The probability density of random walks on fractals, $P(\mathbf{r}, t)$, contains much information about the dynamics as well as about the geometric structure of the fractal (see, for example, section 2.4). In this section numerical data for $P(\mathbf{r}, t)$ and a conjecture about its functional form are presented. We also review the related problem of the distribution of voltage drops on percolation. This problem has recently attracted much attention, and has been suggested to be characterized by a multifractal histogram.

The form of $P(\mathbf{r}, t)$ for percolation clusters or for other fractals has been studied by several authors [111-114] (see section 2.4). In order to study the form of $P(\mathbf{r}, t)$ for $d=2$ percolation clusters, the scaling form (2.19) was first tested. The numerical results were found [114] to be in good agreement with (2.19), and are presented in figure 14. The parallel distribution in chemical space, $P(l, t)$, i.e. the probability density of finding a random walker at a chemical distance $l$ at time $t$ (starting from $l=0$ at $t=0$ ), was also studied. A plausible scaling form for $P(l, t)$, similar to the scaling of $P(\mathbf{r}, t)$ in (2.19) is

$$
P(l, t)=\frac{l^{d_{l}-d}}{t^{d_{\mathrm{s}} / 2}} \phi\left(\frac{l}{t^{1 / d_{\mathrm{w}}^{l}}}\right) .
$$

This form was found [114] too to be in good agreement with numerical data for twodimensional percolation, as seen in figure 15. The question about the specific form of $\Pi(x)$, in (2.19), and $\phi(x)$, in (3.38), is of great interest. A fit to the numerical data of figures 14 and 15 with $\Pi(x)=\exp \left(-x^{u}\right)$ and $\phi(x)=\exp \left(-x^{v}\right)$ is best achieved with $u=1.65 \pm 0.1$ and $v=1.9 \pm 0.1$.

In reference [114] a theoretical relation between $u$ and $v$ is obtained: 


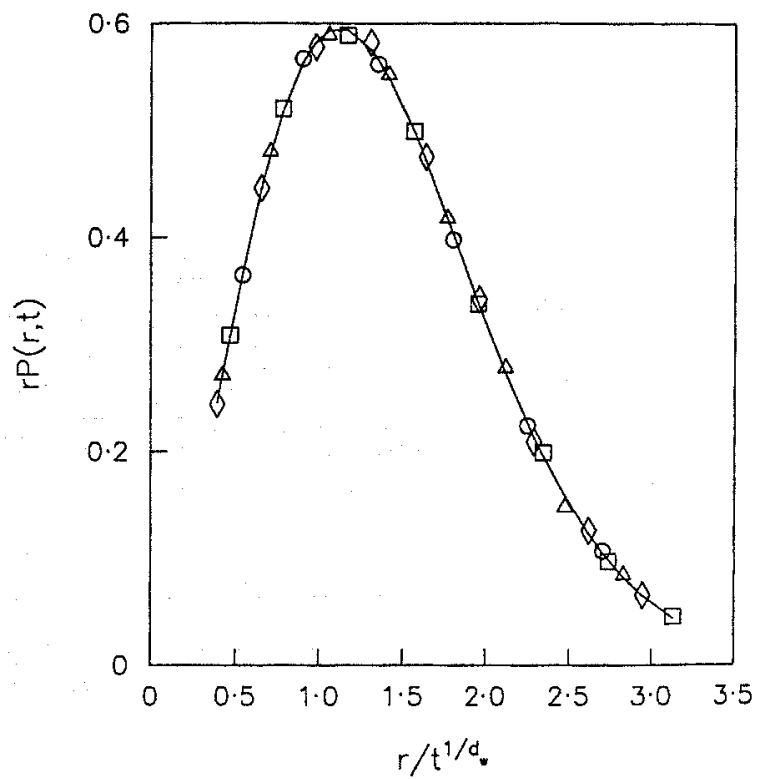

Figure 14. Plot of $r P(r, t)$ against $r / t^{1 / d_{\mathrm{w}}}$ for different values of $r$ and $t$. The different symbols represent different numbers of steps: $\bigcirc, t=1000 ; \square, 1500 ; \triangle, 2000 ; \diamond$, 2500. The range of $r$ is $10 \leqslant r \leqslant 150$. The solid line represents the best fit to (2.19) with $\Pi(x)=\exp \left(-a x^{u}\right)$ and $u=1.65 \pm 0.1$.

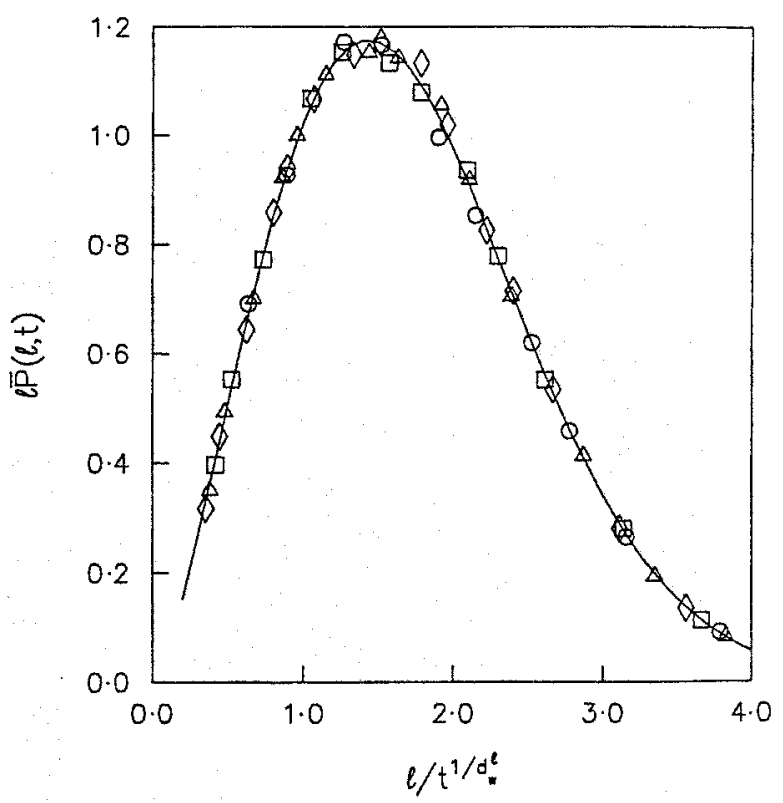

Figure 15. Plot of $l P(l, t)$ against $l / t^{d_{\mathrm{w}}^{l}}$ for different values of $l$ and $t$. The different symbols represent different numbers of steps: $\bigcirc, t=1000 ; \square, 1500 ; \triangle, 2000 ; \diamond, 2500$. The range of $l$ is $10 \leqslant l \leqslant 100$. The solid line represents the best fit to (3.38) with $\phi(x)=\exp \left(-b x^{v}\right)$ and $v=1.9 \pm 0.1$. 


$$
u=v \tilde{\delta} /(v+\tilde{\delta} \tilde{\nu})
$$

Using the relation $\tilde{\delta}=(1-\tilde{\nu})^{-1}$, one obtains

$$
\tilde{\nu}=\frac{1-u^{-1}}{1-v^{-1}} .
$$

This relation is consistent with the numerical values found for $u, v$ and $\tilde{\nu}$ (see section 3.6). The above numerical values for $u$ and $v$ are in disagreement with the prediction of Banavar and Willemson [112] and O'Shaughnessy and Procaccia [111]. They argue that (2.20) holds (i.e. $u=d_{\mathrm{w}}=2.87$ ) for percolation clusters in $d=2$; this is in clear disagreement with the value $u=1.65 \pm 0.1$ of [114]. This value of $u$ is slightly higher than that obtained from (2.18): $u=d_{\mathrm{w}} /\left(d_{\mathrm{w}}-1\right) \approx 1.53$. This parallels the differences found for the measured and predicted values of diffusion on the Sierpinski gasket, discussed in section 2.4.

Substituting $u=d_{\mathrm{w}} /\left(d_{\mathrm{w}}-1\right)$ into $(3.39 b)$ yields

$$
v=\frac{d_{\mathrm{w}}^{l}}{d_{\mathrm{w}}^{l}-1} .
$$

Since $d_{\mathrm{w}}^{l}=\tilde{\nu} d_{\mathrm{w}} \approx 2.54$ (two-dimensional percolation), we obtain $v=1.65$, which is significantly smaller than the measured value $v=1.9 \pm 0.1$.

An interesting related case is the form of $P(l, t)$ for a random walker on a percolation cluster generated on a Cayley tree at criticality. Simulations were performed using the exact enumeration method, and the data are presented in figure 16. In this case we find that $v=\frac{3}{2}$ describes the data best. This is consistent with (3.40) using the known value [57] $d_{\mathrm{w}}^{l}=3$. In section 4 we present other loopless fractal structures for which (3.40) is valid. This leads us to suspect that the equations

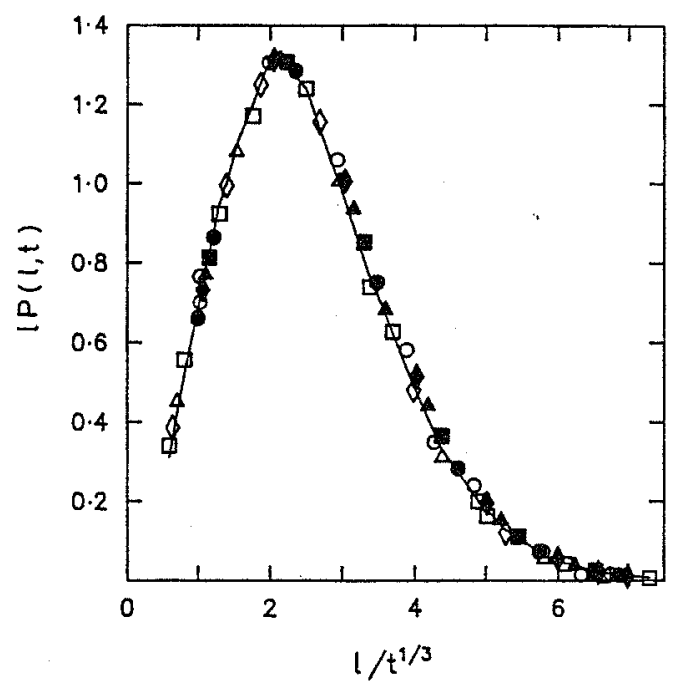

Figure 16. Plot of $l P(l, t)$ as a function of $l / t^{1 / d_{\mathrm{w}}^{l}}$ for different values of $l$ and $t$ for percolation on a Cayley tree. The different symbols specify different numbers of steps: $\bigcirc, t=400 ; \diamond, 800 ;+, 1200 ; \triangle, 1600 ; \square, 2000 ; \times, 2400 ; \nabla, 2800 ; \square, 3600$; 0, 4000. The solid curve represents the best fit to (3.38) and (3.40) with $\phi(x)=\exp \left(-b x^{v}\right)$, using the parameter $d_{l}=2.02 \pm 0.05, d_{\mathrm{s}}=1.35 \pm 0.05$ and $d_{\mathrm{w}}^{l}=$ $3.0 \pm 0.10$. 


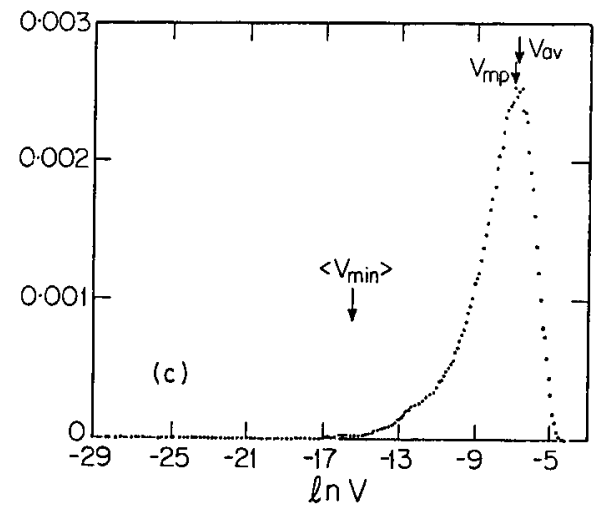

Figure 17. The voltage distribution of a $130 \times 130$ square-lattice random-resistor network at the percolation threshold. (After de Arcangelis et al. [116].)

$u=d_{\mathrm{w}} /\left(d_{\mathrm{w}}-1\right)$ and (3.40) are exact for the case of anomalous diffusion on loopless structures, but only approximate for aggregates with loops.

A related problem that is currently very fashionable is the study of probability densities for which the conventional scaling approach cannot describe the system. Instead, an infinite hierarchy of exponents is needed to characterize the moments of the distribution. Systems with such a property are termed multifractals. The distribution of voltages in a random resistor network [116] is a good example. Suppose that one has a percolation cluster at criticality where each bond is of unit resistance. On applying a voltage on two opposite sites of the cluster, a voltage drop $V_{i}$ results on bond $i$. The distribution of the voltage drops $\left\{V_{i}\right\}$, has a very long tail, and is very different from Gaussian (see figure 17). Let $\alpha=V_{i} / V_{\max }$, where $V_{\max }$ is the largest voltage drop on a bond in the system, and let $n(\alpha)$ be the distribution of $\{\alpha\}$. The maximum voltage $V_{\max }$ occurs on singly connected (red) bonds of the cluster. The $k$ th moment of $\alpha$ is

$$
\alpha_{k} \equiv\left\langle a^{k}\right\rangle=\sum_{\alpha} \alpha^{k} n(\alpha)
$$

Define the hierarchy of exponents $\zeta_{k}$ by

$$
\alpha_{k} \sim L^{\zeta_{k}}
$$

where $L$ is the linear size of the system. Several of the $\zeta_{k}$ can be related to known critical percolation exponents. For example, consider $\alpha_{0}=\sum_{\alpha} n(\alpha) \sim L^{\zeta_{0}}$. Since there is no voltage drop on bonds belonging to dangling ends, $\sum_{\alpha} n(\alpha)$, counts all of the bonds belonging to the backbone. Thus $\alpha_{0} \sim L_{\mathrm{f}}^{d_{\mathrm{f}}^{\mathrm{BB}}}$, and $\zeta_{0}$ is identified as $\zeta_{0}=d_{\mathrm{f}}^{\mathrm{BB}}$. Similarly, the moment $\alpha_{2}$ is proportional to the resistance of the cluster with $\zeta_{2}=\tilde{\mu}+2-d$, and $\alpha_{4}$ describes the fluctuations in the total resistance of the cluster, i.e. the electrical noise [196]. Also, $\alpha_{\infty}$ takes into account the red bonds only, and accordingly $\zeta_{\infty}=1 / \nu$. Similar results were found also by Rammal et al. [196] when studying the properties of resistance noise in random networks. For the case of probability distribution functions that have a scaling property, there exists a gap exponent $\Delta$, such that $\zeta_{k}=k \Delta+\zeta_{0}$ (section 2.4). In contrast, for the random resistor network $n(\alpha)$ does not scale, and no gap exponent is found. Instead, an infinite hierarchy of exponents describe the different moments $\alpha_{k}$. An analogous 
behaviour was found in growth of diffusion-limited aggregation [117-119], in the localization problem [197], in percolation with nonlinear resistors [198], and in diffusion in the presence of random fields (H. Roman, A. Bunde and S. Havlin, preprint). An infinite set of exponents was first suggested by Mandelbrot for the problem of the onset of turbulence [199]. For a recent review on multifractals see Stanley and Meakin [27].

\subsection{Numerical results}

Few of the numerical values of the critical exponents describing the static (geometrical) properties of percolation clusters are known exactly. These include all exponents in $d \geqslant 6$ (mean field) and $\beta$ and $\nu$ for two-dimensional percolation. The rest of the exponents are known chiefly from numerical simulations, from renormalization-group theory and from series expansions. A representative list of the known values for the static critical exponents of percolation is presented in table 2 .

The first numerical estimates [47] for the diffusion exponents $d_{\mathrm{w}}$ and $d_{\mathrm{s}}$ were obtained using short walks of the order of a few hundred steps. Later it was realized by Havlin and Ben-Avraham [80] and by Pandey and Stauffer [81] that the diffusion exponents do not converge for short walks, and walks much longer than $10^{3}$ steps are needed. Results for $d_{\mathrm{w}}$ on large percolation clusters in $d=2$ and $d=3$ are presented in figure 18. Figure 19 shows results for $d_{\mathrm{w}}^{\prime}$ (averages over all clusters) in $d=3$ [81]. In both figures $d_{\mathrm{w}}$ and $d_{\mathrm{w}}^{\prime}$ were calculated from local slopes of $\ln R(N)$ plotted against $\ln N$. It can be seen that the slope changes with $N$, and is smaller for smaller $N$. An efficient numerical method for estimating $\tilde{\mu}$ is by simulating diffusion on the backbone. The conductivity of a percolation cluster is determined solely by the conductivity of the backbone. The backbone is defined as follows. Assume that each bond (or site) in the cluster is a resistor and that an external potential drop is

Table 2. Static exponents for percolation.

\begin{tabular}{|c|c|c|c|c|}
\hline$d$ & $\nu$ & $d_{\mathrm{f}}$ & $d_{\mathrm{f}}^{\mathrm{BB}}$ & $\tilde{\nu}=1 / d_{\min }$ \\
\hline 2 & $\frac{4}{3}$ & $\frac{91}{48}$ & $\begin{array}{c}1.62 \pm 0.02 \\
1.61 \pm 0.01 \\
25 / 16^{\mathrm{c}} \\
{[193]}\end{array}$ & $\begin{array}{l}0.87 \pm 0.02 \\
0.883 \pm 0.003[187] \\
0.870 \pm 0.015^{\mathrm{d}} \\
\quad 16 / 17^{\mathrm{c}}\end{array}$ \\
\hline 3 & $0.88 \pm 0.02$ & $2.51 \pm 0.02$ & $1.74 \pm 0.04$ & $\begin{array}{lr}0.75 & {[175]} \\
0.725 \pm 0.015 & {[181]}\end{array}$ \\
\hline 4 & $\begin{array}{l}0.72 \pm 0.03[181] \\
0.64 \pm 0.02[217] \\
0.62^{\mathrm{a}}[204,205] \\
0.67-0.68^{\mathrm{b}}[206]\end{array}$ & $\begin{array}{l}3.05 \pm 0.05[181] \\
3.12 \pm 0.02[204] \\
3.21 \pm 0.07 \\
3.09^{\mathrm{a}}[204,205]\end{array}$ & $1.9 \pm 0.2$ & $\begin{array}{lll}0.68 & \pm 0.05 & {[177]} \\
0.62 & \pm 0.02 & {[181]}\end{array}$ \\
\hline 5 & $\begin{array}{lr}0.51 \pm 0.05 & {[217]} \\
0.57^{\mathrm{b}} & {[206]} \\
0.56^{\mathrm{a}} & {[204,205]}\end{array}$ & $\begin{array}{l}3.69 \pm 0.02[217] \\
3.54^{\mathrm{a}}[204,205]\end{array}$ & $1.93 \pm 0.16[177]$ & $0.59 \pm 0.05$ \\
\hline 6 & $\frac{1}{2}$ & 4 & 2 & $\frac{1}{2}$ \\
\hline
\end{tabular}

\footnotetext{
a From $\epsilon$-expansion.

${ }^{\mathrm{b}}$ From series expansion.

c From conformal invariance.

d From D. Laidlaw, G. Mackay and N. Jan (preprint, 1987).
} 


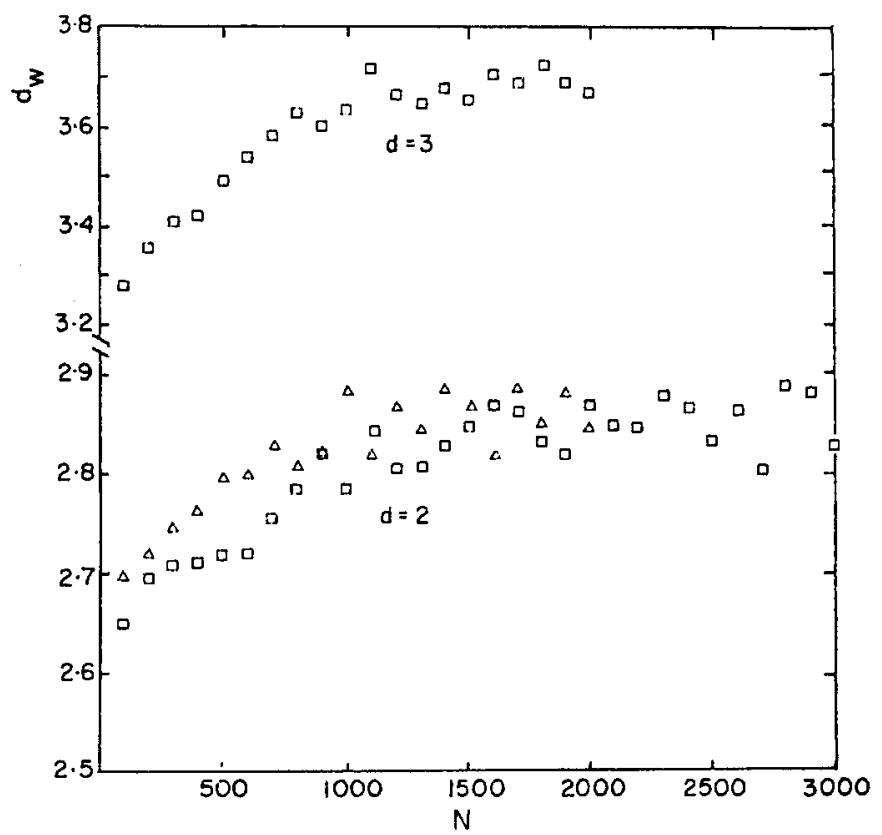

Figure 18. Graph of $d_{\mathrm{w}}$ from the slope of the curve $\ln N$ against $\ln R(N)$. The squares represent the data for diffusion on percolation on a square lattice, and the triangles data for a triangular lattice.

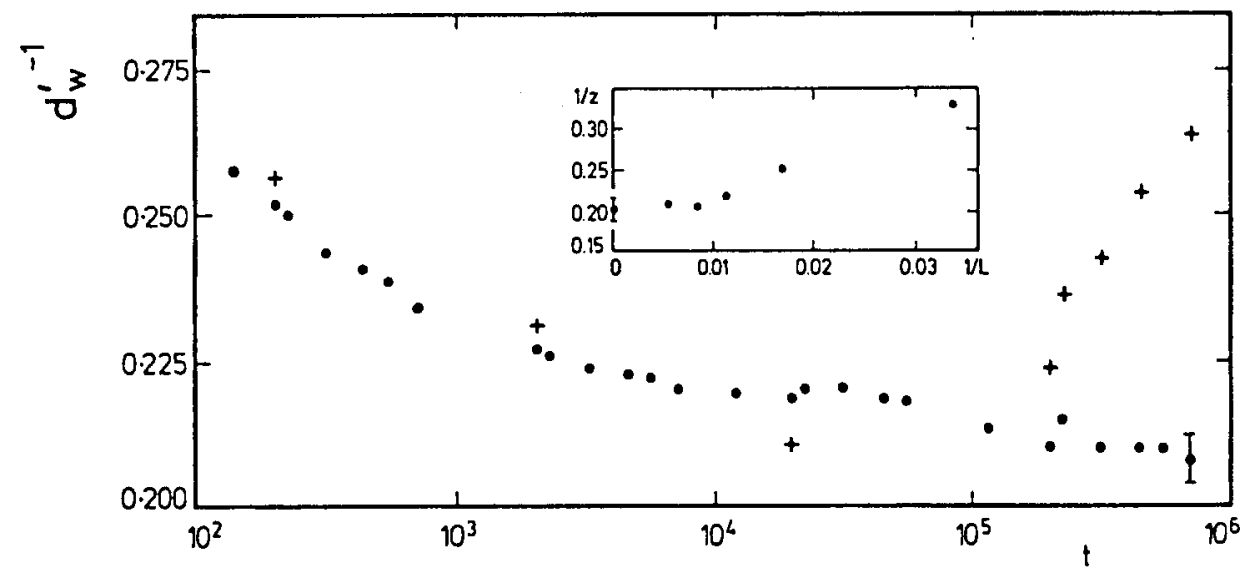

Figure 19. Plot of $1 / d_{\mathrm{w}}^{\prime}$ against time $t$ on a logarithmic time scale. The linear dimension $L$ of the lattice is $180\left(\right.$ ) and $60(+)$. The inset shows the variation of $d_{\mathrm{w}}^{\prime}$ near $t=10^{6}$ with system size L. (After Pandey and Stauffer [81].)

applied at two ends of the cluster. The backbone is the subset of the cluster consisting of all bonds (or sites) through which the current flows (see figure 20). The backbone is thus the structure left when all 'dangling' ends are eliminated from the cluster. Dangling ends are those parts of the cluster that can be disconnected from the cluster by cutting one bond only. The dangling ends do not contribute to the conductivity, since the current does not flow through them. From $d_{\mathrm{w}}=d_{\mathrm{f}}+\tilde{\zeta}$ and $(2.7 a)$, it follows that $d_{\mathrm{w}}^{\mathrm{BB}}=d_{\mathrm{f}}^{\mathrm{BB}}+\tilde{\zeta}$, where $d_{\mathrm{f}}^{\mathrm{BB}}$ and $d_{\mathrm{w}}^{\mathrm{BB}}$ are the fractal 


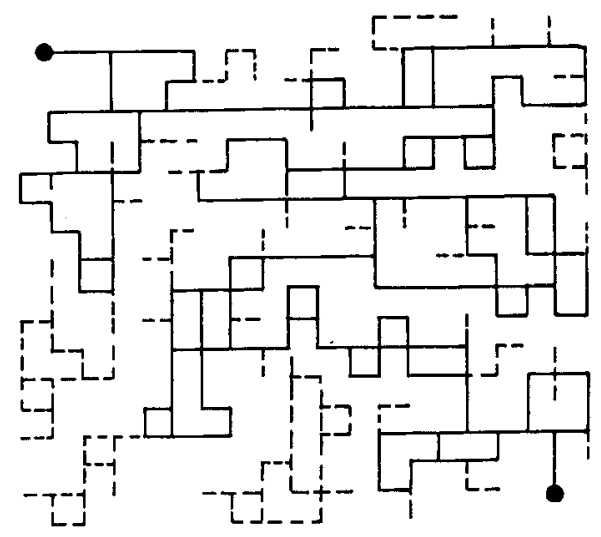

Figure 20. Schematic plot of the backbone of a percolation cluster. The backbone consists of the solid lines, and the dashed lines represent the dangling ends. The voltage drop is applied at the two points represented by the full circles.

dimension and the diffusion exponent of the backbone. The advantage of studying diffusion on the backbone is that it eliminates the need to simulate diffusion on the dangling ends, which is irrelevant to the conductivity, but very time-consuming. Indeed, numerical studies of diffusion on the backbone yield [82] accurate results for $\tilde{\zeta}$ (or $\tilde{\mu}$ ) for percolation. Numerical estimates of $d_{\mathrm{w}}$, as well as more recent numerical values obtained by Majid et al. [83], Bug et al. [200] and others, are presented in table 3. This table also includes numerical estimates for the conductivity exponent $\tilde{\mu}$ (which is related to $d_{\mathrm{w}}$ by $d_{\mathrm{w}}=2+\tilde{\mu}-\beta / \nu$ ), and for $d_{\mathrm{s}}, d_{\mathrm{w}}^{\mathrm{BB}}$ (the anomalous exponent for diffusion on the backbone) and $d_{\mathrm{w}}^{l}$. The numerical estimates of $d_{\mathrm{s}}$ in $d=2$ are inconsistent with the AO conjecture. See, however, the discussion in section 2.3 .

The resistance exponent $\zeta \equiv \tilde{\zeta} \nu=\mu+(2-d) \nu$ was calculated by Harris and Lubensky [215] and by Wang and Lubensky [216] using a renormalization-group $\epsilon$-expansion $(\epsilon=6-d)$ up to second order in $\epsilon$ :

$$
\zeta=1+\frac{\epsilon}{42}+\frac{4 \epsilon^{2}}{2987}+O\left(\epsilon^{3}\right) .
$$

This result yields $=1.167$ for $d=2$ and $\zeta=1.083$ for $d=3$, compared with $\zeta=1.29$ $(d=2)$ and $\zeta=1.12(d=3)$ obtained by numerical simulations. It is interesting to note that real-space renormalization-group results of Bernasconi [201] yield $\mu=$ $1.33 \pm 0.02$ for $d=2$ and $\mu=2.14 \pm 0.02$ for $d=3$, in good agreement with the numerical estimates.

\subsection{Continuum percolation}

Recently, Halperin, Feng and Sen [153] have studied the transport properties near the percolation threshold of a class of 'Swiss-cheese' or random-void continuum models [162]. These models consist of uniform media in which spherical holes are randomly placed. The static percolation exponents, such as $\nu$ and $\beta$, for these continuum models have been confirmed by simulations to be the same as for ordinary lattice percolation [154, 209]. In contrast, Halperin et al. [153] found that the electrical conductivity and the elastic-constant exponents are non-universal, and in continuum percolation they can be quite different from those in discrete-lattice 
Diffusion in disordered media

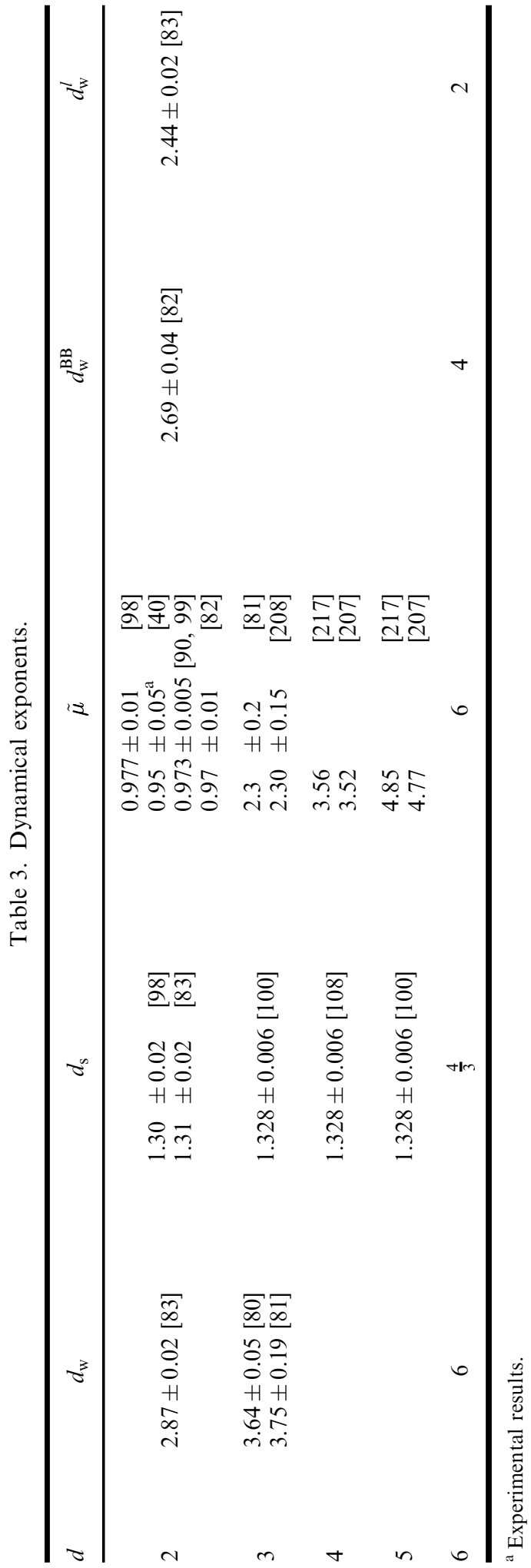


percolation systems. These results have been obtained by mapping [154] the continuum system onto random percolation networks with a distribution of bond conductivities $\{\sigma\}$, (or elasticities)

$$
P(\sigma) \sim \sigma^{-\alpha}, \quad \alpha<1, \quad 0<\sigma \leqslant 1 .
$$

For the random-void model the mapping is achieved with $\alpha=-1$ in $d=2$, and $\alpha=\frac{1}{3}$ in $d=3$, and in general $\alpha=(2 d-5) /(2 d-3)$.

Discrete percolation systems with a power-law distribution of conductivities as in (3.42) have been studied by Kogut and Straley [210], Ben-Mizrahi and Bergman [211], Straley [212] and Sen et al. [155]. In the original work of Kogut and Straley [210], they employed an effective-medium theory on a Cayley-tree model. They suggested that the conductivity exponent depends on $\alpha$, and $\mu(\alpha)=\mu+\alpha /(1-\alpha)$ for $\alpha>0$. Here $\mu$ is the standard conductivity exponent for percolation systems with constant bond conductivities. Ben-Mizrahi and Bergman [211], using an approximate renormalization-grou $\mathrm{p}$ method, found $\mu(\alpha)=A \mu+B \alpha /(1-\alpha)$ for $\alpha>0$, with $A$ and $B$ constants close to unity. Straley [212], using the 'nodes-links-blobs' model for percolation, concluded that $\mu(\alpha)=\max (\mu, 1+(d-2) \nu+\alpha /(1-\alpha))$. Halperin et al. [153] presented arguments that the predictions of [210] and [212] yield upper and lower bounds on $\mu(\alpha)$ respectively, i.e.

$$
1+(d-2) \nu+\frac{\alpha}{1-\alpha} \leqslant \mu(\alpha) \leqslant \mu+\frac{\alpha}{1-\alpha}, \quad 0 \leqslant \alpha \leqslant 1 .
$$

The results obtained for the conductivity of continuum percolation using an $\epsilon$-expansion [156] show a more complicated crossover behaviour. For a detailed discussion see Lubensky and Tremblay [156] and the recent review on the $\epsilon$-expansion results by Harris [213]. Benguigui [214] and C. J. Lobb and M. G. Forrester (preprint, 1987), presented experimental results in $d=2$ that are in good agreement with Halperin et al. [153].

In a recent work, Bunde et al. [158] studied how the diffusion exponents change on the incipient infinite percolation cluster (in $d=2$ ) when the transition rates between nearest-neighbour sites, $\{w\}$, are chosen from a power-law distribution

$$
P(w) \sim w^{-\alpha}, \quad \alpha<1, \quad w \leqslant 1,
$$

analogous to (3.42). For uniform transition rates $(w=1$, corresponding to $\alpha=-\infty)$ it has been shown that $d_{\mathrm{w}}$ is related to $\mu$ via $d_{\mathrm{w}}=2+(\mu-\beta) / \nu$ as in (3.18). Using similar arguments, it can be shown that (3.18) also holds for the general case of random transition rates, where $d_{\mathrm{w}}$ and $\mu$ are replaced by $d_{\mathrm{w}}(\alpha)$ and $\mu(\alpha)$. Thus from (3.43) one obtains bounds on $d_{\mathrm{w}}(\alpha)$ :

$$
d_{\mathrm{f}}+\frac{1}{(1-\alpha) \nu} \leqslant d_{\mathrm{w}}(\alpha) \leqslant d_{\mathrm{w}}+\frac{\alpha}{(1-\alpha) \nu}, \quad 0 \leqslant \alpha \leqslant 1,
$$

where $d_{\mathrm{f}}=d-\beta / \nu$ is the fractal dimension of the incipient infinite percolation cluster. Since the geometrical properties of the cluster are not affected by the conductivity assigned to the bonds, it follows that the chemical-diffusion exponent $d_{\mathrm{w}}^{l}(\alpha)$ is related to $d_{\mathrm{w}}(\alpha)$ through

$$
\frac{d_{\mathrm{w}}^{l}(\alpha)}{d_{\mathrm{w}}(\alpha)}=\frac{d_{\mathrm{f}}}{d_{\mathrm{f}}}=\tilde{\nu} \equiv \frac{1}{d_{\min }} .
$$

This yields 


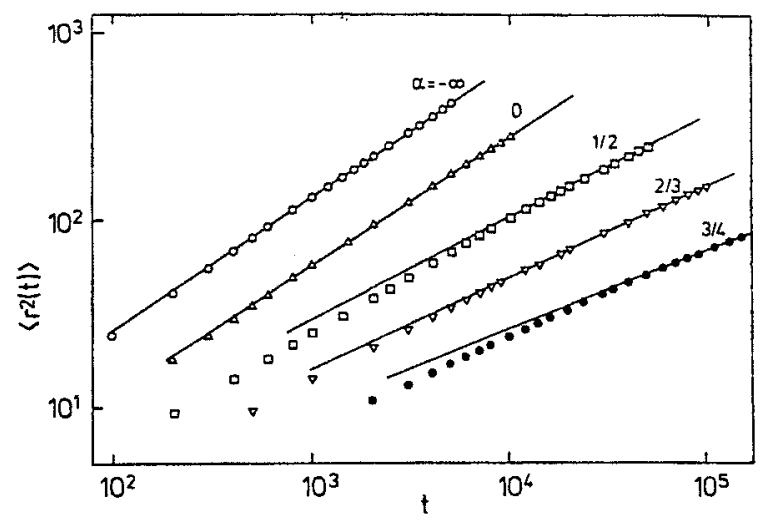

Figure 21. Plot of $\left\langle r^{2}(t)\right\rangle$ against $t$ for various $\alpha$. For the calculations, clusters were generated up to 150 shells (typically 10000 sites) using the Leath algorithm [184]. To determine $\left\langle r^{2}(t)\right\rangle$, exact numeration for the 'blind ants' was used (section A.2 of the appendix). Each value of $\alpha$ represents an average over 400 configurations.

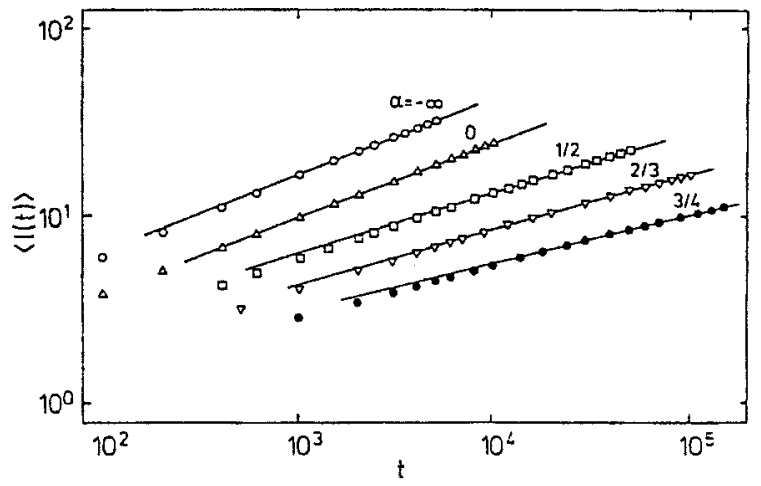

Figure 22. Plot of $\langle l(t)\rangle$ against $t$ for various $\alpha$, from the same data as in figure 21 .

$$
d_{l}+\frac{1}{(1-\alpha) \nu_{l}} \leqslant d_{\mathrm{w}}^{l} \leqslant d_{\mathrm{w}}^{l}+\frac{\alpha}{(1-\alpha) \nu_{l}}
$$

where $\nu_{l}=\nu d_{\mathrm{f}} / d_{l}$ is the 'chemical correlation exponent', characterizing the scaling of the correlation length in chemical space $\xi_{l} \sim\left(p-p_{\mathrm{c}}\right)^{-\nu_{l}}$ (see reference [57]).

Relations (3.45) and (3.47) are easily accessible to a direct numerical test. To this purpose the exact-enumeration method was applied (see section A.2 of the appendix).

The results for $\left\langle r^{2}(t)\right\rangle$ and $\langle l(t)\rangle$ are shown [158] in figures 21 and 22 for various values of $\alpha$. From the asymptotic slopes, one obtains the diffusion exponents $d_{\mathrm{w}}(\alpha)$ and $d_{\mathrm{w}}^{l}(\alpha)$, which are presented in table 4 . The results strongly support the inequalities (3.45) and (3.47), and thus also support the scaling arguments leading to these relations. Note that for small $\alpha, d_{\mathrm{w}}(\alpha)$ is closer to the upper bound, while for large $\alpha, d_{\mathrm{w}}(\alpha)$ is closer to the lower bound. Very recently, simulations of diffusion in $d=2$ and $d=3$ continuum percolation systems have been performed (J. Petersen, H. E. Roman, A. Bunde and W. Dieterich, 1987, preprint). The results support the non-universality and are in good argreement with (3.43) and (3.45); but if the steplength of the walker is finite a new universality class is found, even for $d=2$. For a 
Table 4. The diffusion exponents $d_{\mathrm{w}}$ and $d_{\mathrm{w}}^{l}$ extracted from figures 21 and 22 for five values of $\alpha$, compared with the upper and lower bounds (3.45).

\begin{tabular}{lcccc}
\hline$\alpha$ & \multicolumn{3}{c}{ Upper-bound } \\
$d_{\mathrm{w}}(\alpha)$ & $\begin{array}{c}\text { Lower-bound } \\
d_{\mathrm{w}}(\alpha)\end{array}$ & $d_{\mathrm{w}}^{l}(\alpha)$ \\
\hline$-\infty$ & $d_{\mathrm{w}}(\alpha)$ & 2.87 & 2.65 & $2.47 \pm 0.04$ \\
0 & $2.85 \pm 0.04$ & 2.87 & 2.65 & $2.50 \pm 0.05$ \\
$\frac{1}{2}$ & $2.90 \pm 0.05$ & 3.62 & 3.40 & $3.13 \pm 0.06$ \\
$\frac{2}{3}$ & $3.60 \pm 0.10$ & 4.37 & 4.15 & $3.15 \pm 0.08$ \\
$\frac{3}{4}$ & $4.13 \pm 0.10$ & 5.12 & 4.90 & $4.00 \pm 0.15$ \\
\hline
\end{tabular}

recent review on transport properties of percolation (Swiss-cheese model) see Feng et al. [160].

Machta et al. [157] studied transport on a hierarchical resistor network model. This model is very useful for describing the transport properties of the backbone of percolation clusters [116]. The conductivities $\{\sigma\}$ were chosen from a power-law distribution (3.42). Machta et al. applied a renormalization-grou p transformation using an ansatz about the form of the distribution of resistances. Their conclusion for the conductivity exponent was that

$$
\mu(\alpha)= \begin{cases}\mu & \left(\mu>(d-2) \nu+\frac{1}{1-\alpha}\right), \\ (d-2) \nu+\frac{1}{1-\alpha} & \left(\mu<(d-2) \nu+\frac{1}{1-\alpha}\right) .\end{cases}
$$

Weiss and Havlin [159] presented a rigorous proof of (3.48) for the hierarchical resistor network by solving the recursion relation for the distribution of the conductivities in a real-space renormalization-group analysis. As noted above, the results for $d_{\mathrm{w}}(\alpha)$ for percolation are closer to the upper bound for small $\alpha$, while they are closer to the lower bound for large $\alpha$. This can be partly understood from the result (3.48) and also from recent findings by Lubensky and Tremblay [156]. For an earlier study of one-dimensional continuum percolation see Domb [163].

\section{Diffusion on loopless structures}

The study of loopless aggregates is considerably simpler than the general case of aggregates with loops. Indeed, several models could be solved exactly because of the simplification and lack of correlations once loops are neglected. A well known example is percolation on the Cayley tree (mean-field percolation). There exist many physical realizations and models of aggregates in which loops can be neglected. The simplest is perhaps the linear polymer modelled by self-avoiding walks [71]. Other examples are branches polymers modelled by lattice animals [218, 219], chemically linear branched polymers [220], aggregate s such as those modelled by DLA [63, 221], and growth models for trees [87, 222]. It has been realized that all these random structures are of a fractal nature and possess anomalous transport properties. In this chapter we review the physics of transport phenomena in loopless structures. One of the most satisfying results arising from the simplicity of loopless aggregates is a 
general exact relation between transport (dynamical) exponents $\left(d_{\mathrm{w}}, d_{\mathrm{w}}^{l}\right.$ and $\left.d_{s}\right)$ and structural (static) exponents $\left(d_{l}\right.$ and $\left.d_{\mathrm{f}}\right)$. For the general case of aggregates with loops, such a relation has not yet been found (see section 3), in spite of the large effort extended.

\subsection{Relation between transport exponents and structural exponents}

In the following we present a scaling argument [52] that relates the diffusion exponents on loopless fractals to the structural exponents. The resistance exponent of a cluster is defined by the scaling of the resistance $\varrho(R)$ between two sites separated by a distance $R$ :

$$
\varrho(R) \sim R^{\tilde{\zeta}}
$$

Since loops can be neglected, the shortest (chemical) path is the only path connecting any two sites. Thus, the resistance scales as the chemical distance $l$ between these points

$$
\varrho \sim l \sim S^{1 / d_{l}} \sim R^{d_{\mathrm{f}} / d_{l}}=R^{d_{\min }},
$$

from which it follows that

$$
\tilde{\zeta}=d_{\mathrm{f}} / d_{l}=d_{\min } .
$$

For calculating the diffusion exponent, we use the Einstein relation $(2.7 a)$, $d_{\mathrm{w}}=d_{\mathrm{f}}+\tilde{\zeta}$ and

$$
d_{\mathrm{w}}=d_{\mathrm{f}}\left(1+\frac{1}{d_{l}}\right) .
$$

Thus both transport exponents $\tilde{\zeta}$ and $d_{\mathrm{w}}$ are determined by the structural exponents $d_{\mathrm{f}}$ and $d_{l}$.

The chemical-distance diffusion exponent $d_{\mathrm{w}}^{l}=\tilde{\nu} d_{\mathrm{w}}$ is

$$
d_{\mathrm{w}}^{l}=d_{l}+1
$$

and the fracton dimensionality $d_{\mathrm{s}}=2 d_{\mathrm{f}} / d_{\mathrm{w}}=2 d_{l} / d_{\mathrm{w}}^{l}$ is

$$
d_{\mathrm{s}}=\frac{2 d_{l}}{d_{l}+1} .
$$

Note that both $d_{\mathrm{w}}^{l}$ and $d_{\mathrm{s}}$ depend only on $d_{l}$, and not on $d_{\mathrm{f}}$.

The number of growing sites in the diffusion front $G(l)$ on a loopless fractal can be calculated using the Rammal-Toulouse equation (2.11), $\mathrm{d} S / \mathrm{d} t \sim G / S$. Substituting for the number of distinct sites $S \sim t^{d_{\mathrm{s}} / 2} \sim t^{d_{l} / d_{\mathrm{w}}^{l}}$, we find that the front of the walk (i.e. the set of growth sites $G(l))$ on a fractal scales as

$$
G(l) \sim l^{d_{l}-1} .
$$

That is, the front of diffusion is a spherical cut of the fractal in the chemical-distance space (not in the Pythagorean space; see also the discussions in references $[95,96]$ ). It is in this sense that the chemical distance is a natural length parameter for the study of diffusion. In contrast, using the $R$-metric (Pythagorean space), the front of diffusion is not a simple spherical cut, but rather a complex fractal structure (see the discussion following (2.11)).

\subsection{Diffusion on branched polymers modelled by lattice animals (LA)}

The structural properties of large branched polymers in a dilute solution have long been of interest. Lattice animals have been found to be a useful model for such 
polymers. Zimm and Stockmayer [218] found the fractal dimension for lattice animals generated on a Cayley tree, $d_{\mathrm{f}}=4$. This was later recognized [219] to be exact for spatial dimensions $d$ above $d_{\mathrm{c}}=8$. Today, the exact results for three [223] and four [224] dimensions, $d_{\mathrm{f}}=2(d=3)$ and $d_{\mathrm{f}}=\frac{12}{5}(d=4)$ are known. These results characterize spatial properties of the structure. However, many physical properties, such as transport and elasticity, strongly depend on the intrinsic topological structure of the aggregate. For this reason intrinsic topological exponents such as $d_{l}$ and $\tilde{\nu}$ are of interest. By using two methods to generate ensembles of clusters, the series-expansion method [225] and the enrichment method [226], the values of $d_{l}, \tilde{\nu}$ and $d_{\mathrm{f}}$ were estimated [52] for lattice animals in $d=2,3$ and 4 dimensions. The numerical values of the different exponents are given in table 5 . In figure 23 a comparison of $d_{l}$ for LA with $d_{l}$ for percolation clusters is presented. A Flory-type theory was derived [183] for $\tilde{\nu}$ for LA,

$$
\tilde{\nu}=\frac{5}{d+2}
$$

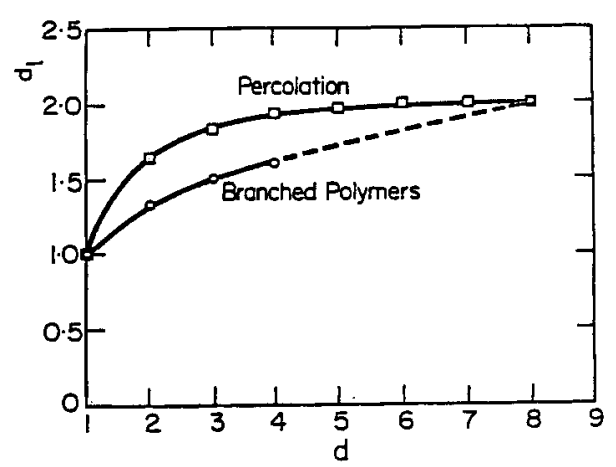

Figure 23. Comparison of the results for $d_{l}$ of lattice animals with the results for $d_{l}$ of percolation clusters. (After reference [52].)

Table 5. Summary of the values for the different exponents of LA discussed in the text.

\begin{tabular}{|c|c|c|c|c|c|c|}
\hline$d$ & $d_{\mathrm{f}}$ & & $d_{l}$ & $d_{\mathrm{w}}$ & $\tilde{\zeta}=d_{\mathrm{f}} / d_{l}$ & $d_{\mathrm{s}} / 2=d_{\mathrm{f}} / d_{\mathrm{w}}$ \\
\hline 1 & 1 & & 1 & 2 & 1 & $\frac{1}{2}$ \\
\hline 2 & $\begin{array}{l}1.56 \\
1.55 \pm 0.05\end{array}$ & $\begin{array}{l}{[249]} \\
{[226]}\end{array}$ & $1.33 \pm 0.02[52]$ & $\begin{array}{l}2.71 \pm 0.10^{\mathrm{b}} \\
2.78 \pm 0.08 \quad[52] \\
2.6 \pm 0.3 \quad[51]\end{array}$ & $1.17 \pm 0.05^{\mathrm{c}}$ & $0.56 \pm 0.03^{d}$ \\
\hline 3 & $2^{\mathrm{a}}$ & [223] & $1.47 \pm 0.04[52]$ & $\begin{array}{l}3.36 \pm 0.10^{\mathrm{b}} \\
3.37 \pm 0.10 \quad[50] \\
3.4 \pm 0.4 \quad[51]\end{array}$ & $1.36 \pm 0.04^{\mathrm{c}}$ & $0.59 \pm 0.02^{\mathrm{d}}$ \\
\hline 4 & $2.4^{\mathrm{a}}$ & [224] & $1.61 \pm 0.06[52]$ & $3.89 \pm 0.06^{\mathrm{b}}$ & $1.49 \pm 0.05^{\mathrm{c}}$ & $0.62 \pm 0.01^{\mathrm{d}}$ \\
\hline 8 & $4^{\mathrm{a}}$ & [218] & {$[52]$} & $6^{\mathrm{b}}$ & $2^{\mathrm{c}}$ & $\frac{2}{3}^{\mathrm{d}}$ \\
\hline
\end{tabular}

a Exact result.

${ }^{b}$ From (4.4).

c From (4.3).

d From (4.6). 


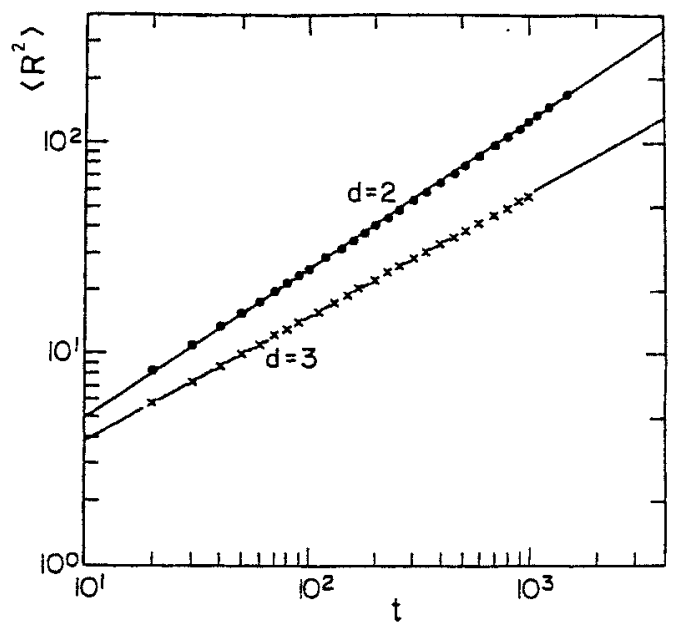

Figure 24. The mean-square displacement $\left\langle R^{2}(t)\right\rangle$ for random walks on LA. The slopes yield $d_{\mathrm{w}}=2.78 \pm 0.08(d=2)$ and $3.37 \pm 0.10(d=3)$.

yielding $d=3$ as the lower critical dimension and $d=8$ as the upper critical dimension. However, (4.7) has little success in predicting the numerical values for $\tilde{\nu}$.

Numerical simulations of diffusion on lattice animals were performed by Wilke et al. [51] and Havlin et al. [52]. In figure 24 we present the results by Havlin et al., which were obtained by using the exact enumeration method (see section A.2 of the appendix). These results are within the error bars of Wilke et al., obtained by Monte Carlo simulations. From the slopes in figure 24 one finds $d_{\mathrm{w}}=2.78 \pm 0.08(d=2)$ and $d_{\mathrm{w}}=3.37 \pm 0.10(d=3)$. This is to be compared with $d_{\mathrm{w}}=2.6 \pm 0.3(d=2)$ and $d_{\mathrm{w}}=3.4 \pm 0.4(d=3)$ found in reference [51]. Since it is well established [219] that for lattice animals loops can be neglected, it is expected that (4.3)-(4.6) are valid. In table 5 we present the values for $d_{\mathrm{w}}$ obtained using these equations. Note the good agreement between the values of $d_{\mathrm{w}}$ obtained from the prediction of (4.4) and from numerical simulations. Wilke et al. [51] suggested a generalization of the scaling theory for diffusion on percolation of section 3.3, which also includes lattice animals:

$$
\left\langle R^{2}\right\rangle \sim R_{\mathrm{s}}^{2}\left(\frac{t}{S^{d_{\mathrm{w}} / d_{\mathrm{f}}}},\left(p-p_{\mathrm{c}}\right) S^{\sigma}\right) .
$$

Here $\sigma=1 / \beta \delta=(1+1 / \delta) / \mathrm{d} \nu$. Equation (4.8) is the percolation analogue of the dynamical scaling [227].

\subsection{Diffusion on DLA clusters}

The diffusion-limited-aggregation (DLA) model of colloids and dendritic growth was introduced by Witten and Sander [63]. In this model a seed particle is fixed at the origin and particles are released one after the other from a circle enclosing the cluster. Each particle moves in a random-walk fashion until it reaches a neighbouring site of the aggregate and becomes part of the growing cluster. It is accepted that the cluster formed is a fractal object having a self-similarity problem in all length scales. The fractal dimensions of these clusters [228, 229] and $d_{\mathrm{f}}=1.68 \pm 0.05$ in $d=2$ and $d_{\mathrm{f}}=2.5 \pm 0.06$ in $d=3$. For recent reviews see references [8, 11, 230]. There is a striking resemblance between a simulated DLA (figure 25) and the fractal 


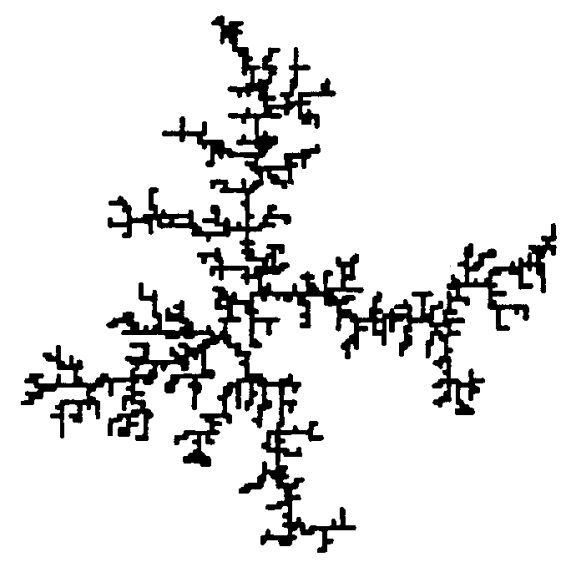

Figure 25. A typical DLA cluster.

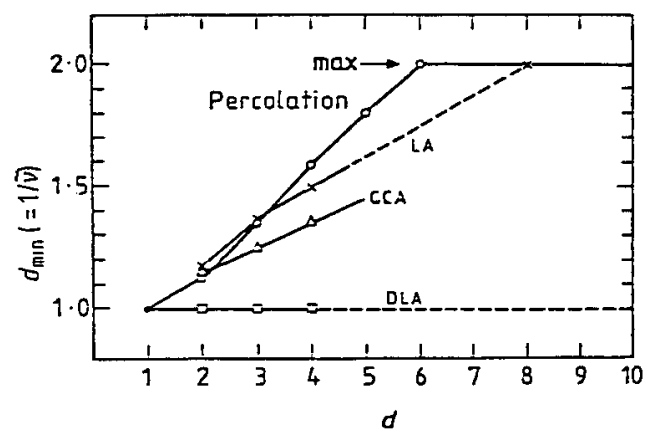

Figure 26. Dependence of $1 / \tilde{\nu}$ on the dimension of space $d$. The data on lattice animals and percolation clusters are from references $[52,57,179]$. The data on DLA and CCA are from [84]. Note that $1 / \tilde{\nu}$ converges to the value of 2 for all models above the critical dimension $d_{\mathrm{c}}$ for all of the models. The only exception is DLA, for which $\tilde{\nu}=1$, within the limits of accuracy, even up to $d=4$. This suggests that there is no upper critical dimension for DLA.

structure of some real physical systems, such as sputter-deposited thin films of $\mathrm{NbGe}_{2}$ [231]. Other experimental realizations of DLA have been found in aggregation of copper on the cathode of an electrolyte [232], in the electrostatic analogue of a diffusing field [233, 234] and in viscous fingers in hydrodynamics [30, 235]. It can be seen from figure 25 that large loops are rare in the structure, and for this reason it is in general accepted that loops can be neglected.

It has been found numerically by Meakin et al. [84] that, in contrast with other aggregates such as LA, cluster-cluster aggregation (CCA) [236, 237] and percolation clusters, the DLA has the peculiar property that

$$
d_{l} / d_{\mathrm{f}}=\tilde{\nu}=1 \quad \text { for } d=2,3,4
$$

In LA, CCA and percolation clusters, $\tilde{\nu}=1$ in $d=1$, and $\tilde{\nu} \rightarrow \frac{1}{2}$ when $d$ approaches the critical dimension (see figure 26 and table 6).

The result $\tilde{\nu}=1\left(d_{\mathrm{f}}=d_{\mathrm{f}}\right)$ for DLA has striking implications. First, it provides numerical support to the idea that there is no upper critical dimension for DLA [63, 
Table 6. Summary of the result for DLA and CCA. After [84].

\begin{tabular}{llllll}
\hline & \multicolumn{2}{c}{ DLA } & & \multicolumn{2}{c}{ CCA } \\
\cline { 2 - 3 } \cline { 5 - 6 }$d$ & $d_{\min }=1 / \tilde{\nu}$ & $d_{l}$ & & $d_{\min }=1 / \tilde{\nu}$ & $d_{l}$ \\
\hline 2 & $1.0 \pm 0.02$ & $1.69 \pm 0.05$ & & $1.15 \pm 0.04$ & $1.22 \pm 0.02$ \\
3 & $1.02 \pm 0.03$ & $2.3 \pm 0.2$ & & $1.25 \pm 0.05$ & $1.42 \pm 0.02$ \\
4 & $1.00 \pm 0.04$ & $3.3 \pm 0.2$ & & $1.35 \pm 0.05$ & $1.55 \pm 0.05$ \\
\hline
\end{tabular}

238]. This follows from the fact that $\tilde{\nu}$ does not approach the mean-field value $\tilde{\nu}=\frac{1}{2}$ as $d$ increases. Secondly, it provides predictions for the diffusion on DLA clusters. It has been shown earlier [52] that if loops are irrelevant then (4.4) and (4.6) are valid. If $d_{l}=d_{\mathrm{f}}$ then, for DLA, in all diemsnions $d$, we have

$$
\left.\begin{array}{l}
d_{\mathrm{w}}=d_{\mathrm{f}}+1, \\
d_{\mathrm{s}}=2 d_{\mathrm{f}} /\left(d_{\mathrm{f}}+1\right) .
\end{array}\right\}
$$

Note also that in this case $d_{\mathrm{w}}$ and $d_{\mathrm{s}}$ depend on $d_{\mathrm{f}}$ alone.

A direct test of (4.10) is provided by extensive simulations of diffusion performed by Meakin and Stanley [221] on DLA clusters. They find

$$
d_{\mathrm{w}}= \begin{cases}2.56 \pm 0.10 & (d=2) \\ 3.33 \pm 0.25 & (d=3)\end{cases}
$$

and

$$
d_{\mathrm{s}}= \begin{cases}1.2 \pm 0.1 & (d=2), \\ 1.3 \pm 0.1 & (d=3),\end{cases}
$$

which is in good agreement with (4.10).

\subsection{Diffusion on comb-like structures}

Although there has been considerable interest in the problem of anomalous diffusion on fractal structures, most results are known only from numerical simulations or scaling theories. The simplest model that yields non-trivial results and can still be solved rigorously [239]-[242] for $P(\mathbf{r}, t)$, is diffusion on comb-like structures such as in figures 27 and 28. In spite of their apparent simplicity, comblike structures are reasonable models for disordered media. The delay of a random walker usually caused by dangling ends and bottlenecks is modelled by the time spent on the teeth of the comb, and the transport along the backbone is non-trivial, and even anomalous.

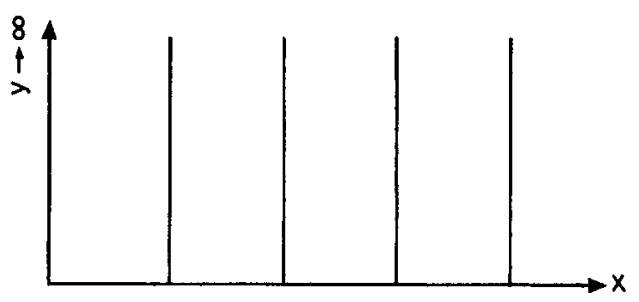

Figure 27. Two-dimensional comb with infinitely long teeth along the $y$-direction. 


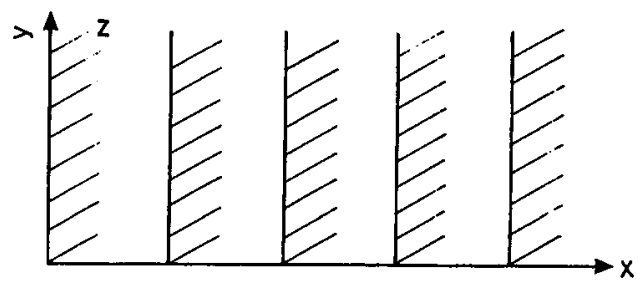

Figure 28. Three-dimensional comb with infinitely long teeth along the $y$-and $z$-directions.

In the following we present the case of combs with an infinite length of the teeth [239]. A general discussion of comb-like structures with different distributions of teeth lengths is presented in sections 5 and 6 . Consider the comb in figure 27. It is expected that a random walker will have different properties in the horizontal and vertical directions. Since the random walk consists of a series of steps in the $y$ direction followed by a series of steps in the $x$-direction, and so forth, the motion along the $x$-axis can be regarded as a discrete analogue of a continuous-time random walk (CTRW) [14, 55]. The corresponding distribution of waiting times was shown [239] to be $\psi(t) \sim t^{-1 / 2}$. It follows from the general theory of continuous-time random walks $[14,55,239,243,244]$ that anomalous diffusion occurs along the backbone, with a mean-square displacement

$$
\left\langle x^{2}\right\rangle \sim t^{2 / d_{\mathrm{w}}}, \quad d_{\mathrm{w}}=4 .
$$

The number of distinct sites visited increases as

$$
\langle s(t)\rangle \sim t^{d_{\mathrm{s}} / 2} .
$$

The probability to return to the origin scales as

$$
P_{0}(t) \sim t^{-d_{\mathrm{s}} / 2}, \quad d_{\mathrm{s}} / 2=\frac{3}{4}
$$

The anomalous diffusion found along the $x$-axis is due to the average infinite sojourn of the walker on the fingers of the comb. It should be noted that the expected number of distinct sites visited along the $x$-axis scales as [239]

$$
\left\langle S^{x}(t)\right\rangle \sim t^{1 / 4}
$$

The probability density of a walker to be in site $(x, y)$ at time $t$ was shown to be [245]

$$
P(x, y, t)=\frac{A}{t^{3 / 4}} \exp \left[-a\left(\frac{x}{t^{1 / 4}}\right)^{3 / 4}-\frac{b y^{2}}{t}\right] .
$$

For the three-dimensional comb of figure 28 the results are [239]

and [245]

$$
\left\langle x^{2}\right\rangle \sim t^{1 / 4}, \quad\left\langle y^{2}\right\rangle \sim t^{1 / 2}, \quad\left\langle z^{2}\right\rangle \sim t
$$

$$
P(x, y, z, t)=\frac{A}{t^{7 / 8}} \exp \left[-a\left(\frac{x}{t^{1 / 8}}\right)^{8 / 7}-b\left(\frac{y}{t^{1 / 4}}\right)^{4 / 3}-\frac{c z^{2}}{t}\right] .
$$

We emphasize that the results of (4.13)-(4.19) are exact asymptotically for $t \rightarrow \infty$.

In the general case of CTRW with a waiting-time distribution $\psi(t) \sim t^{-(1+\gamma)}$, $0<\gamma<1$, anomalous diffusion $\left\langle x^{2}\right\rangle \sim t^{2 / d_{\mathrm{w}}}$ with $d_{\mathrm{w}}=2 / \gamma$ takes place $[243,244]$. In this case the probability density $P(x, t)$ is of the form [245] 


$$
P(x, t)=\frac{A}{t^{d_{\mathrm{s}} / 2}} \exp \left[-a\left(\frac{x}{t^{1 / d_{\mathrm{w}}}}\right)^{d_{\mathrm{w}} /\left(d_{\mathrm{w}}-1\right)}\right] .
$$

One can put the results of (4.14) and (4.16) in the common notation used in the literature. The anisotropy of the comb models has to be taken into account. For example, for the comb of figure 27 the diffusion exponent along the $x$-direction is $d_{\mathrm{w}}^{x}=4$, and along the $y$-direction it is $d_{\mathrm{w}}^{y}=2$. The fracton dimension $d_{\mathrm{s}}$, defined in (4.14), must also be decomposed into two components. Thus from scaling

$$
\langle S(t)\rangle=\left\langle S^{x}(t)\right\rangle\left\langle S^{y}(t)\right\rangle \equiv t^{d_{\mathrm{s}}^{x} / 2} t^{d_{\mathrm{s}}^{y} / 2},
$$

from which it follows that

$$
\frac{d_{\mathrm{s}}}{2}=\frac{d_{\mathrm{s}}^{x}}{2}+\frac{d_{\mathrm{s}}^{y}}{2}=\frac{d_{\mathrm{f}}^{x}}{d_{\mathrm{w}}^{x}}+\frac{d_{\mathrm{f}}^{y}}{d_{\mathrm{w}}^{y}}=\frac{1}{4}+\frac{1}{2}=\frac{3}{4} .
$$

Here $d_{\mathrm{f}}^{x}=d_{\mathrm{f}}^{y}=1$ are the fractal dimensions of the backbone and the teeth of the comb respectively. As can be seen from (4.17), (4.19) and (4.20), the density distribution is not Gaussian, which is consistent with the form suggested in sections 2.4 and 3.5. It is also different from forms of $P(\mathbf{r}, t)$ suggested by Banavar and Willemson [112], Ohtsuki and Keyes [246] and O'Shaughnessy and Procaccia [111].

A more interesting case is the family of one-dimensional combs for which the teeth length $l$ follow a power-law distribution

$$
P(l) \sim l^{-(1+\gamma)} .
$$

For this case the CTRW model is not exact, since each tooth has a different length. A self-consistent scaling approach [291] that takes the memory into account yields (see section 5.5)

$$
\begin{gathered}
d_{\mathrm{w}}=4 /(1+\gamma), \\
d_{\mathrm{s}}=\frac{1}{4}(3-\gamma)
\end{gathered}
$$

Note that the case $\gamma=0$ represents a comb with infinitely long teeth, and (4.24) and (4.25) reduce to (4.13) and (4.15). The case $\gamma=1$ is equivalent to a comb with teeth of finite length, and its transport properties are regular.

In deriving (4.4)-(4.6), we calculated the resistance exponent $\tilde{\zeta}$ from the scaling of the resistance between two sites on the substrate. In general, $\tilde{\zeta}$ should be calculated from the scaling of the resistance between two hyperplanes cutting the substrate. For finitely ramified fractals, such as those studied in this section there is no difference between the resistance measured between two sites or between two hyperplanes, justifying our approach (see also references [69, 77, 78]). The case of diffusion on infinitely ramified loopless structures was studied by Dhar and Ramaswamy [87] and Havlin et al. [86, 222, 247, 248]. These two groups used different approaches, and disagree in their results. In order to clarify this problem, more intensive theoretical and numerical studies have to be done.

\section{Diffusion on random and hierarchical structures}

The general problem of diffusion in a disordered medium can be described by a master equation for the probability distribution of a random walker on a lattice. The 
(a)

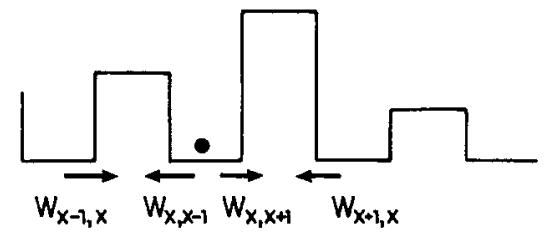

(b)

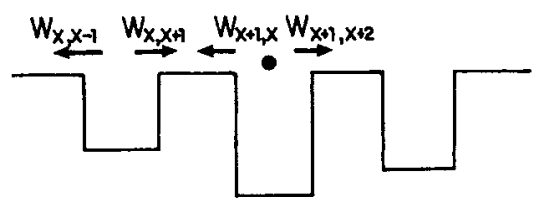

Figure 29. Random barriers $(a)$ and wells $(b)$ in one dimension. For barriers, the transition probabilities for hopping across a barrier to the right or to the left are equal: $w_{x-1, x}=w_{x, x-1}$. For wells, the transition probabilities for getting out of a well (to either side) are equal: $w_{x, x-1}=x_{x, x+1}$.

transition rates between neighbouring sites are random, and their distribution is determined by the specific model studied. In particular, one-dimensional systems have been studied extensively [4, 252-257]. These systems are described by the master equation

$$
\frac{\mathrm{d} P(x, t)}{\mathrm{d} t}=w_{x, x+1}[P(x+1, t)-P(x, t)]+w_{x, x-1}[P(x-1, t)-P(x, t)],
$$

where $P(x, t)$ is the probability for the walker to be at site $x$ at time $t$, and $w_{x, x \pm 1}$ are the transition rates from site $x$ to $x \pm 1$. There is a distinction between the cases [4] of barriers and of wells. The case of barriers is defined by $w_{x, x+1}=w_{x+1, x}$ (and in general $w_{x, x+1} \neq w_{x, x-1}$ ), whereas wells are defined by $w_{x, x+1}=w_{x, x-1}$ (and in general $\left.w_{x, x+1} \neq w_{x+1, x}\right)$. As can be seen from figure 29, the above definitions do indeed correspond respectively to the cases of a particle hopping across barriers or out of wells. Recently there has been growing interest in the problem of a power-law distribution of transition rates because of its relevance to various physical systems, such as the following.

(i) The temperature dependence of the dynamical conductivity exponent observed in the one-dimensional superionic conductor hollandite can be explained by a model with a power-law distribution of transition rates [37].

(ii) Continuum random systems such as the Swiss-cheese model can be mapped [153] onto random percolation networks with a power-law distribution of bond conductivities. Employing the partly 'one-dimensional' nature of the backbone of the percolation infinite cluster, bounds on the transport exponents have been derived [153, 155, 158, 160, 210, 212, 258]. (For a more detailed discussion see section 3.7.)

(iii) The problem of biased diffusion in random structures such as the random comb or the percolation system can be modelled [252, 259, 260] by biased diffusion in a linear chain with a power-law distribution of transition rates. In this case, the power-law exponent depends on the bias field, and a dynamical phase transtion occurs in which the drift velocity changes from non-zero to zero upon crossing a critical value of the bias field. 


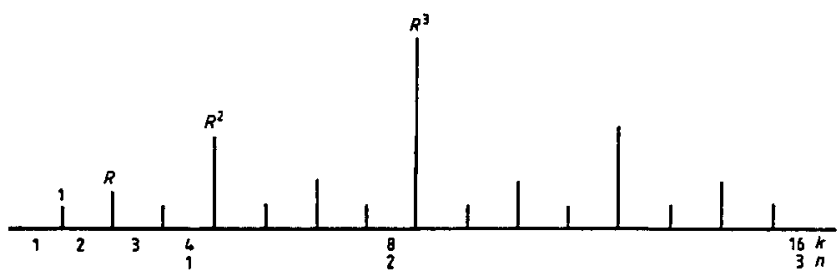

(a)

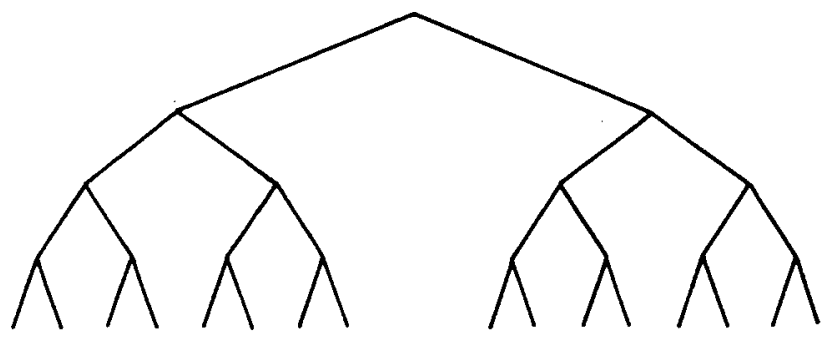

(b)

Figure 30. (a) Hierarchical-potentials structure. The particle can hop from a cell to its nearest-neighbour cell with transition rates that depend on the potentials. The index $k$ represents the cell number and $n$ represents supercells determined by $k=2^{n+1}$. (b) The ultrametric tree of coordination number $z=3$ from which the hierarchical structure in $(a)$ is generated.

(iv) Anomalous relaxation in spin glasses can be interpreted in terms of stochastic motion (in phase space) with a power-law distribution of transition rates [261, 262].

A deterministic realization of a one-dimensional system with a power-law distribution of transition probabilities is provided by hierarchical structures, such as that shown in figure $30(a)$. The hierarchical structure of figure $30(a)$ is constructed iteratively. The $n$th iteration is obtained by doubling the structure of the $(n-1)$ th iteraction and adding in the centre a barrier of height $R^{n+1}$. (The first iteration is a single barrier of height $R^{0}=1$.) This structure is strongly related to the tree structure of figure $30(b)$. The height of each barrier between two sites is $R^{k-1}$, where $k$ is their closest common ancestor level. The tree of figure $30(b)$ is an example of an ultrametric space. Ultrametricity is a mathematical concept that has recently been found useful in various physical contexts. For a recent review of ultrametricity in physics see reference [263].

In the following we review the problem of transport in one-dimensional systems with a distribution of transition rates. We also review the generalization of the onedimensional problem to higher dimensions and to fractals. The techniques developed for the solution of the transport problem in one dimension are then applied to the problem of comb-like structures with various distributions of teeth lengths.

\subsection{Transport in one-dimensional systems with a power-law distribution of conductivities}

The problem of transport in one-dimensional systems with a broad distribution of conductivities has been studied in recent years by several authors (see, for 
example, references $[4,210,212,256,258])$. For a power-law distribution of bond conductivities

$$
P(\sigma) \mathrm{d} \sigma=(1-\alpha) \sigma^{-\alpha} \mathrm{d} \sigma, \quad \alpha<1, \quad 0 \leqslant \sigma \leqslant 1,
$$

the transport exponent $\tilde{\zeta}$ and $d_{\mathrm{w}}$ depend sensitively [4] on $\alpha$ :

$$
\tilde{\zeta}= \begin{cases}1 & (\alpha \leqslant 0), \\ (1-\alpha)^{-1} & (\alpha>0),\end{cases}
$$

and

$$
d_{\mathrm{w}}= \begin{cases}2 & (\alpha \leqslant 0) \\ \frac{2-\alpha}{1-\alpha} & (\alpha>0)\end{cases}
$$

In the following a simple scaling theory to derive (5.3) is presented. This approach proves to be useful for the solution of other related problems, as shown later in this section.

Consider a one-dimensional chain of length $L$ consisting of $L$ bonds whose conductivities $\sigma_{i}$ are distributed according to (5.2). The total conductivity $\Sigma$ is given by

$$
\Sigma^{-1} \equiv \sum_{i=1}^{L} 1 / \sigma_{i}
$$

When $L \gg 1$ one can pass to the continuum limit:

$$
\Sigma^{-1} \approx L \int_{\sigma_{\min }}^{1} \frac{1}{\sigma} P(\sigma) \mathrm{d} \sigma=\frac{1-\alpha}{\alpha} L\left(\sigma_{\min }^{-\alpha}-1\right) \sim \begin{cases}L & (\alpha<0), \\ L \ln \sigma_{\min } & (\alpha=0), \\ L \sigma_{\min }^{-\alpha} & (\alpha>0) .\end{cases}
$$

Here $\sigma_{\min }$ is a cut-off corresponding to the smallest conductivity of the (finite) system. Equation (5.5) describes a dynamical phase transition. For $\alpha<0$ transport is normal, and for $\alpha>0$ transport is anomalous. The transition, at $\alpha=0$, is characterized by a logarithmic correction.

In order to estimate $\sigma_{\min }$, we choose a random variable $u(0 \leqslant u \leqslant 1)$ distributed uniformly so that $P(\sigma) \mathrm{d} \sigma=\mathrm{d} u$, i.e. $u=\sigma^{-\alpha+1}$. The expected minimum value of $u$ is $u_{\min }=1 / L$ (since there are $L$ conductivities), and thus

$$
\sigma_{\min }=L^{-1 /(1-\alpha)} \text {. }
$$

Substitution of (5.6) into (5.5) yields

$$
\varrho \equiv \Sigma^{-1} \sim \begin{cases}L & (\alpha<0) \\ L \ln L & (\alpha=0) \\ L^{1 /(1-\alpha)} & (\alpha>0) .\end{cases}
$$

and $(5.3 a)$ is recovered.

To derive $(5.3 b)$, one can use the Einstein relation $d_{\mathrm{w}}=\tilde{\zeta}+d_{\mathrm{f}}\left(\right.$ here $\left.d_{\mathrm{f}}=1\right)$. An alternative way is to start with the results for diffusion in one-dimensional random systems, with a power-law distribution of transition rates $\left\{w_{i}\right\}$. This approach was developed by Machta [265], Zwanzig [266] and more recently for higher-dimensional systems by Kundu and Phillips [267]. Replacing $w_{i} \equiv \sigma_{i}$, the problem of random conductivities is exactly mapped to the problem of diffusion in the presence of 
random barriers. The mean-square displacement $\left\langle x^{2}(t)\right\rangle$ is related to the number $N$ of distinct sites visited and to the transition rates $\left\{w_{i}\right\}$. For large $t$ and $N$ [265-267]

$$
D^{-1}=\frac{t}{\left\langle x^{2}\right\rangle}=\frac{1}{N} \sum_{i=1}^{N} \frac{1}{w_{i}} .
$$

This equation can be interpreted as representing the average time spent by the random walker at a site of the system in two equivalent ways. Identifying $L$ with $\left\langle x^{2}\right\rangle^{1 / 2}=N$, from the approach of (5.4)-(5.6), (5.3 b) is rederived.

\subsection{One-dimensional systems with a power-law distribution of potential barriers or wells and hierarchical structures}

The distinction between barriers and wells in one-dimensional systems is not relevant for most transport properties [257, 269]. This is because a random walker must cross all the barriers, or wells, between any two visited sites. In contrast, in higher dimensions a random walker can get around barriers, and the distinction between barriers and wells is important. In the following we discuss one-dimensional systems, treating the cases of barriers and wells as equivalent. However, the probability of returning to the origin, $P_{0}(t)$, is different [270] for barriers and wells even in $d=1$, and is treated separately. See also recent work by J. W. Haus and K. W. Kehr (preprint, 1987).

Consider a one-dimensional system with potential barriers or wells distributed according to

$$
\psi(V)=\left(\gamma V_{0}^{\gamma}\right) V^{-(1+\gamma)}, \quad V_{0} \leqslant V<\infty, \quad \gamma>0,
$$

where $V$ is the size of the barrier or well (it may be regarded as a potential). There exist several possibilities of associating transition probabilities $\left\{w_{i}\right\}$ with this distribution of potentials. When the transition probability $w$ is inversely proportional to $V$, one obtains

$$
P(w)=\psi(V) \frac{\mathrm{d} V}{\mathrm{~d} w} \sim w^{-\alpha}, \quad \alpha=1-\gamma,
$$

and the problem is identical with the one discussed in the previous section, with the transport results of $(5.3 a, b)$.

A deterministic system that has similar anomalous transport properties to the random system discussed above is the one-dimensional lattice with potentials distributed in a hierarchical way, shown in figure $30(a)$. The potentials are given by

$$
V_{k}=R^{l}, \quad R \geqslant 1,
$$

where $l$ is given by the solution of

$$
k\left(\bmod 2^{l}\right)=2^{l-1}, \quad l>0 \text { integer. }
$$

It can be shown that the potentials are algebraically distributed according to $\psi(V) \sim V^{-(1+\ln 2 / \ln R)}$, as in (5.9). In the case that the transition rates $w$ are inversely proportional to $V$, one obtains $p(w) \sim w^{-\alpha}$, with $\alpha=1-\ln 2 / \ln R$. Thus, naively, we may assume that the results of $(5.3 a, b)$ are valid. On the other hand, this is not obvious, because of the deterministic nature of the hierarchical structure. It is, indeed, a surprise that the results of $(5.3 a, b)$ are valid. This has been shown, using renormalization-grou $p$ techniques [262, 271-274]. A simple renormalization-grou p approach was recently suggested [274] to calculate the diffusion exponent $d_{\mathrm{w}}$. The set 
of cells $\{k\}$ is renormalized to supercells $\{n\}$, with $k=2^{n+1}$ (see figure $30(a)$ ). For these new supercells, one can write a recursion relation

$$
\frac{1}{w_{n}}=\frac{2}{w_{n-1}}+R^{n},
$$

where

$$
\frac{1}{w_{n}}=\sum_{k=1}^{2^{n-1}} \frac{1}{w_{k}} .
$$

Using (5.8), (5.13) and (5.14), we have

$$
D^{-1}=\frac{1}{2} \sum_{n=0}^{\bar{n}}\left(\frac{R}{2}\right)^{n}
$$

where $\bar{n}$ represents the span of the walk, $\left\langle x^{2}\right\rangle^{1 / 2} \equiv x \sim k$, and is given by

$$
x=2^{\bar{n}+1} \text {. }
$$

Thus, in the limit of large $x$, i.e. $\bar{n} \gg 1$,

$$
\frac{t}{\left\langle x^{2}\right\rangle} \equiv D^{-1}= \begin{cases}(2-R)^{-1} & (R<2), \\ \frac{1}{2}\left(\frac{1}{2} R\right)^{\bar{n}-1} & (R \geqslant 2),\end{cases}
$$

and there is a dynamical phase transition [262]

$$
d_{\mathrm{w}}= \begin{cases}2 & (R<2), \\ 1+\frac{\ln R}{\ln 2} & (R \geqslant 2) .\end{cases}
$$

This is indeed the result obtained from $(5.3 b)$, with $\alpha=1-\ln 2 / \ln R$. Numerical results using the exact enumeration method (see section, A.2 of the appendix) supporting (5.18) are shown in figure 31. When regarding the potentials as

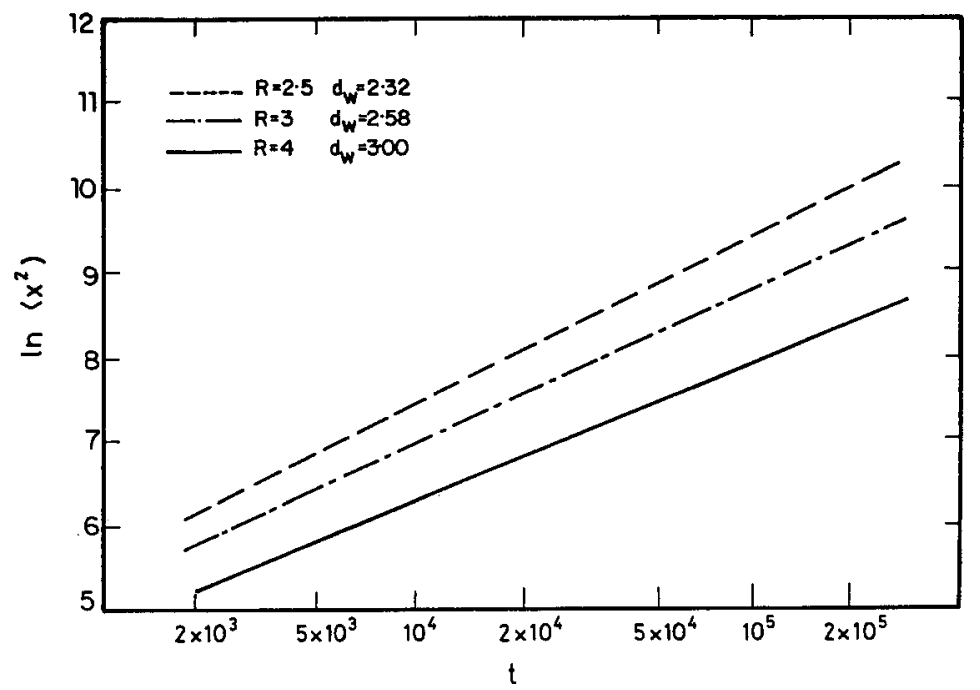

Figure 31. Plot of $\ln \left\langle x^{2}\right\rangle$ against $\ln t$ for the hierarchical structure of figure $30(a)$ for $R=2.5,3,4$. The diffusion exponent $d_{\mathrm{w}}$ calculated from the slopes agrees with (5.18). 
conductivities, the resistance exponent is obtained from the equivalence between conductivities and transition rates (section 5.1)

$$
\tilde{\zeta}= \begin{cases}1 & (R<2), \\ \frac{\ln R}{\ln 2} & (R \geqslant 2) .\end{cases}
$$

The hierarchical structure of figure $30(a)$ can be generalized by constructing it from a tree (figure $30(b)$ ) with coordination number $z$. In this case, $\gamma$ of (5.9) generalizes to $\gamma=\ln (z-1) / \ln R$. The probability of returning to the origin, $P_{0}(t)$, is different for the cases of barriers and wells. A heuristic argument yields [270]

$$
P_{0}(t) \sim \begin{cases}t^{-1 / d_{\mathrm{w}}} & \text { for barriers } \\ t^{-\left(d_{\mathrm{w}}-1\right) / d_{\mathrm{w}}} & \text { for wells. }\end{cases}
$$

Numerical results for the hierarchical structure of figure $30(a)$ support (5.20). Blumen, Zumofen and Klaffer [275] studied numerically the related problem of the distribution of the number of sites visited by a walker on hierarchical structures.

Another case of interest is when the transition probability $w$ is given by the Boltzmann factor

$$
w=\exp (-\beta V)
$$

where $\beta=1 / k T$. The distribution of transition rates $P(w)$ is

$$
P(w)=\psi(V) \frac{\mathrm{d} V}{\mathrm{~d} w}=\frac{\gamma V_{0}^{\gamma}}{\beta} \frac{1}{w(\ln w)^{1+\gamma}} .
$$

This distribution arises naturally in the context of biased diffusion on combs (see reference [276] and section 6.2). To calculate the diffusion properties we use (5.8):

$$
D^{-1}=\left\langle\frac{1}{w}\right\rangle=\frac{\gamma V_{0}^{\gamma}}{\beta} \int_{w_{\min }}^{w_{0}} \frac{\mathrm{d} w}{w^{2}(\ln w)^{1+\gamma}} \sim w_{\min }^{-1}\left|\ln w_{\min }\right|^{-(1+\gamma)} .
$$

In order to estimate $w_{\min }$, we first calculate $V_{\max }$, which represents the maximum barrier or well in a system of linear size $x$. We choose a random variable $u$ $(0 \leqslant u \leqslant 1)$ distributed uniformly such that $P(V) \mathrm{d} V=\mathrm{d} u$, i.e. $u=\left(V / V_{0}\right)^{-\gamma}$. The expected minimum value of $u$ is $u_{\min }=1 / x$, and thus $V_{\max }=V_{0} x^{1 / \gamma}$, from which it follows that

$$
w_{\min }=\exp \left(-\beta V_{0} x^{1 / \gamma}\right) .
$$

For $x \rightarrow \infty$ we obtain from (5.23)

$$
D^{-1}=\frac{t}{\left\langle x^{2}\right\rangle} \sim \exp \left(\beta V_{0} x^{1 / \gamma}\right)
$$

and the leading behaviour for the displacement $x$ is anomalously slow, and has a logarithmic time dependence [276]

$$
x \sim(\ln t)^{\gamma}, \quad 0<\gamma<\infty .
$$

Again, if one associates a transition rate $w=\exp (-\beta V)$ with the potentials of the hierarchical model of figure $30(a)$ then the same result as in (5.26), with $\gamma=\ln 2 / \ln R$, is obtained. This can be shown from a rigorous renormalizationgroup approach [277]. 
When the transition rates $\left\{w_{i}\right\}$ in (5.22) are replaced by conductivities $\left\{\sigma_{i}\right\}$, the resistance of the system $\varrho=\sum_{i} \sigma_{i}^{-1}$ scales as

$$
\varrho \sim \exp \left(L^{1 / \gamma}\right)
$$

where $L$ is the size of the system.

It should be noted that a logarithmic behaviour of the displacement similar to (5.26) was found by Sinai [278]:

$$
x=\left\langle x^{2}\right\rangle^{1 / 2} \sim(\ln t)^{2} .
$$

His model consists of a linear lattice $(d=1)$ in which a random bias field $E_{i}$ is applied on each bond. When the $\left\{E_{i}\right\}$ are symmetrically distributed around $\left\langle E_{i}\right\rangle=0$ the net bias field applied on a region of size $x$ scales as $E \equiv \sum_{i=1}^{x} E \sim x^{1 / 2}$. To overcome the bias and exit this region, it takes a random walker a time $t$ of order $\exp (E) \sim \exp \left(x^{1 / 2}\right)$, and (5.28) follows. The Sinai problem is equivalent to a system with a power-law distribution of potentials, (5.9), with $\gamma=2$. In this case, the maximum characteristic potential barrier within a region of size $x$ scales as $V_{\max } \sim x^{1 / 2}$, and $x \sim(\log t)^{2}$. Thus a power-law distribution of potential barriers with $\gamma=2$ is analogous to the random-field Sinai model, and yields the same results.

The density distribution $P(x, t)$ for a random walker in the Sinai problem has been studied recently by H. Roman, A. Bunde and S. Havlin (preprint). It was found numerically that, while the average density distribution and consequently the moments $\left\langle x^{n}\right\rangle$ scale with a single exponent, it requires an infinite hierarchy of exponents to characterize the fluctuations in $P(x, t)$. An analytical approach for the average of $P(0, t)$ was given by Bauchaud et al. [268]. It is interesting to note that numerical simulations performed by Pandey [279] in the analogous $d=3$ randomfield system indicate that for long times the random walker approaches a drift velocity, i.e. $r \sim t$. A perturbation expansion for diffusion in the presence of a random field was carried out by Winter et al. [280].

The case of non-symmetrical distribution of local bias fields $\left\{E_{i}\right\}$ was studied by Derrida and Pomeau [281]. They considered a model where $E_{i}$ is the field acting on bond $i$ to the right and $1-E_{i}$ to the left, with the distribution of $E_{i}$

$$
\phi\left(E_{i}\right)=c \delta\left(E_{i}-E\right)+(1-c) \delta\left[E_{i}-(1-E)\right] .
$$

For $\frac{1}{2}<c<E$ the mean displacement is [281]

$$
\left\langle x^{2}(t)\right\rangle^{1 / 2} \sim t^{\nu}
$$

with $\nu=\ln [c /(1-c)] / \ln [E /(1-E)]$. Note that the Sinai model [278] is the special case when there is no global bias.

\subsection{Transport in strips $(n \times \infty)$ and in $d \geqslant 2$ with random barriers and wells}

The results derived in sections 5.1 and 5.2 are for single one dimensional chains. As mentioned above, they are the same for energy barriers and for potential wells. In the case of strips $(n \times \infty)$, i.e. $n$ linear chains connected in parallel (figure $32(a)$ ), the transport properties for barriers are different from those of wells. The reason for this difference is that a random walker at site $i$ steps to its nearest neighbours with equal probabilities in the case of potential wells (regardless of their depths), whereas in the case of potential barriers the probability is largest to step across the lowest barrier. Thus, while in the wells system the walker will often be trapped by the deeper wells, 
(a)

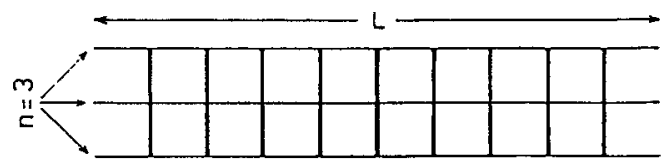

(b)

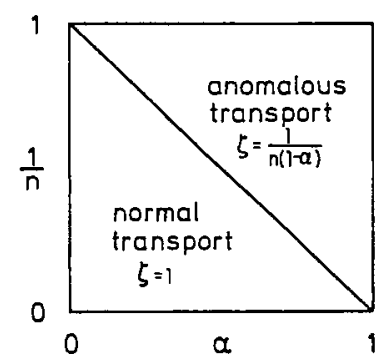

Figure 32. (a) Strip of length $L$, consisting of $n=3$ connected linear chains. The vertical bonds are perfect conductors while the horizontal bond conductivities are distributed as in (5.2). (b) Phase diagram for strips as in (a). The regions of normal and anomalous transport are shown.

in a system of barriers the higher barriers are systematically avoided. Note that in the case of a single one-dimensional chain this difference does not exist, since the walker cannot bypass the higher barriers. Thus, it is argued that for the same distribution of wells and barriers, the mean-square displacement satisfies the inequality

$$
\left\langle r^{2}(t)\right\rangle_{\text {barriers }} \geqslant\left\langle r^{2}(t)\right\rangle_{\text {wells }} .
$$

Consider the case of a strip consisting of $n$ connected parallel chains of length $L$, with a power-law distribution of potentials on the horizontal bonds [277, 282]. For simplicity, we first treat the case where there is no potential associated with the vertical bonds. If the transition rates are inversely proportional to the potential, $w \sim 1 / V$, then the case of barriers is mapped to that of a strip with random bond conductivities (see section 5.1). For this case it was found [282] that the transport exponents in $(5.3 a, b)$ are not universal, but depend sensitively on $n$. For finite $n$ there exists a critical value of $\alpha, \alpha_{\mathrm{c}}=1-1 / n$ (a phase diagram is presented in figure $32(b))$. For $\alpha \leqslant \alpha_{\mathrm{c}}$ the resistance exponent and the diffusion exponent $d_{\mathrm{w}}$ retain their classical values $\tilde{\zeta}=1$ and $d_{\mathrm{w}}=2$. For $\alpha>\alpha_{\mathrm{c}}$ both exponents vary continuously with $n$ and $\alpha$ :

$$
\left.\begin{array}{rl}
\tilde{\zeta} & =\frac{1}{n(1-\alpha)} \quad\left(\alpha>\alpha_{\mathrm{c}}=1-1 / n\right), \\
d_{\mathrm{w}} & =1+\frac{1}{n(1-\alpha)} \quad\left(\alpha>\alpha_{\mathrm{c}}\right) .
\end{array}\right\}
$$

A recent study by Webman (1987, private communication) yields similar results. If the vertical bonds have the same power-law distribution as the horizontal bonds, (5.2) then (5.32) represent rigorous lower bounds. It is argued [283], however, that (5.32) are also correct when all bonds, vertical and horizontal, follow the same 
distribution law (5.2). Recent numerical simulations by H. Taitelbaum (1987, unpublished) for $n=2$ support this claim.

This rich behaviour of transport-dependent properties on the width $n$ of the strips, found in the case of potential barriers (or conductivities), is not found in the case of potential wells. As noted earlier, there is a finite a priori probability of entering a well regardless of its depth, and therefore the transport properties of $n$ parallel chains are qualitatively equal to those of a single one-dimensional chain.

The case of transition probabilities $\left\{w_{i}\right\}$, Boltzmann-distributed, $w=\exp (-\beta V)$, has been analysed [277]. The same transport laws are found for $n$-connected chains and for a single chain, (5.26).

Systems of a power-law distribution of barriers and wells in $d \geqslant 2$ can also be studied using the techniques of sections 5.1 and 5.2. For the case of barriers, with $w \sim 1 / V$, transport is regular [257]. This can be seen from (5.32) on taking the limit $n \rightarrow \infty$. For wells it has been shown [257, 284, 285] that $d=2$ is an upper critical dimension. The diffusion exponent is given by [257, 284, 285]

$$
d_{\mathrm{w}}=\frac{2}{1-\alpha}, \quad d \geqslant d_{\mathrm{c}}=2, \quad 0 \leqslant \alpha<1,
$$

independent of $d(d \geqslant 2)$. This result is the same as that obtained [243, 250, 286-289] for a continuous-time random walk (CTRW) with a waiting-time distribution $P(t) \sim t^{-(1+\alpha)}$. The exponent $d_{\mathrm{w}}$ of $(5.3 \mathrm{~b})$ and $(5.33)$ was also derived by Machta [285] using a real-space renormalization-grou p method. Numerical simulations [284] support these results. For barriers with transition probabilities that are Boltzmanndistributed, $w=\exp (-\beta V)$, it has been shown [277] that there exists a critical value of $\gamma, \gamma_{\mathrm{c}}$. For $\gamma<\gamma_{\mathrm{c}}$ a slow logarithmic transport results, and the mean-square displacement depends strongly on the dimension $d$ (for all $d$ ):

$$
r^{1 / \gamma-(d-1)} \sim \ln t\left(\gamma<\gamma_{\mathrm{c}}=\frac{1}{2(d-1)}\right) .
$$

For $\gamma>\gamma_{\mathrm{c}}$ the diffusion is regular [277], $\left\langle r^{2}\right\rangle \sim t$. For $d=1, \gamma_{\mathrm{c}} \rightarrow \infty$; that is, the logarithmic time dependence is valid for all $\gamma$, as found earlier in (5.26). Note that there is no upper critical dimension $d_{\mathrm{c}}$ as in (5.33). In the case of wells, with Boltzmann-distributed transition probabilities, the mean-square displacement is given by [276]

$$
\left\langle r^{2}\right\rangle^{1 / 2} \sim(\ln t)^{\gamma / 2}, \quad d \geqslant d_{\mathrm{c}}=2 .
$$

These results for $d \geqslant 2$ are obtained with a similar approach to that of sections 5.1 and 5.2. The assumption that (5.8) is valid for systems in $d \geqslant 2$ is also used [267]. One also has to take into account that in $d \geqslant 2$ the number $N$ of distinct sites visited by an ordinary random walker, which appears in (5.8), is propotional to the meansquare displacement $\left\langle r^{2}\right\rangle$.

The various results for barriers and wells in $d=1$, strips, and for $d \geqslant 2$, for the different distributions discussed in this chapter are summarized in table 7.

Other potential distributions might be of interest. For example, an exponential distribution of wells with a Boltzmann distribution of transition probabilities has been studied [284]. For the distribution

$$
P(V)=\frac{1}{V} \exp \left(-\frac{V}{\bar{V}}\right)
$$


Diffusion in disordered media

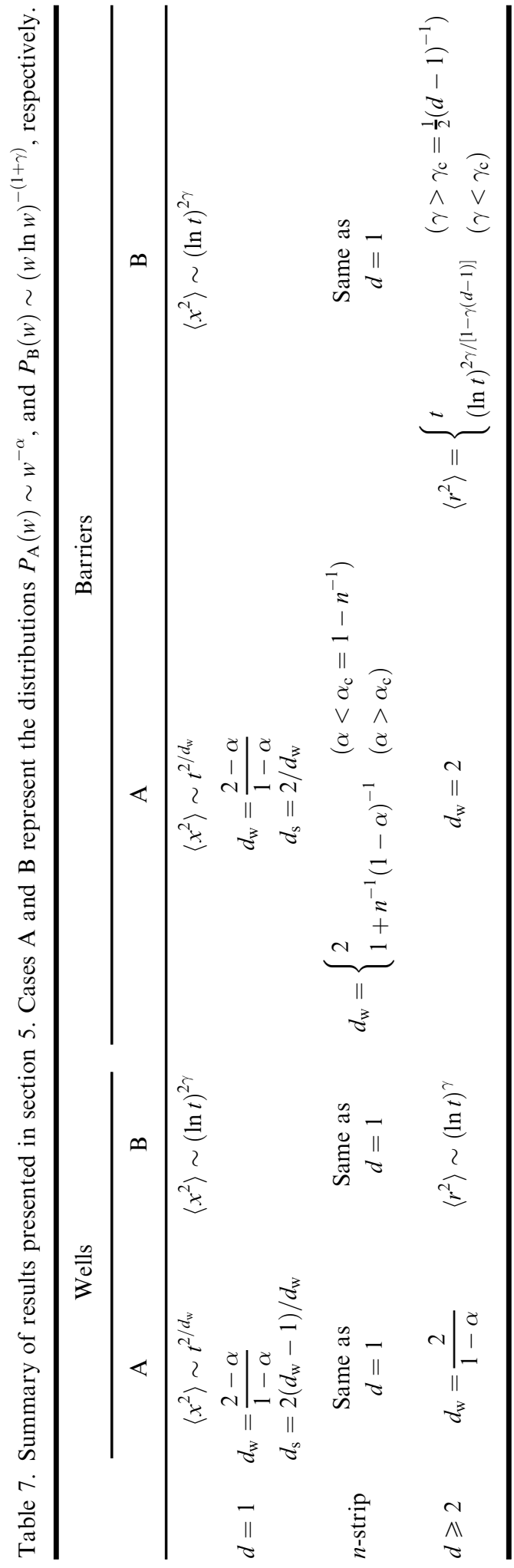


there is a phase transition in the dynamics. For $T>T_{\mathrm{c}}=\bar{V}$ diffusion is normal, and for $T<T_{\mathrm{c}}$ the diffusion is anomalous:

$$
d_{\mathrm{w}}=\left\{\begin{array}{ll}
\frac{T+T_{\mathrm{c}}}{T} & (d=1) \\
\frac{2 T_{\mathrm{c}}}{T} & (d \geqslant 2)
\end{array} \quad\left(T<T_{\mathrm{c}}\right) .\right.
$$

The transport exponents depend on the temperature $T$ and on the dimension $d$ of the system.

\subsection{Potential barriers and wells on fractals}

The case of a power-law distribution of barriers or conductivities on fractals is related to continuum percolation, and was discussed in section 3.7. This problem is not completely solved. The only fractal substrates studied have been percolation clusters and hierarchical lattices. For percolation clusters bounds have been found for the transport exponents [153], but no exact results have been obtained. However, for some hierarchical lattices the transport exponents are known exactly [157, 159].

The transport problem on fractals with a distribution of wells seems to be simpler. This is due to the fact that a random walker enters a well with an equal $a$ priori probability, regardless of the depth of the wells. A distinction must be made between fractals with a fracton dimensionality $d_{\mathrm{s}}$ greater or less than two. For fractals with $d_{\mathrm{s}}<2$ diffusion is recurrent, and the number of distinct sites visited up to time $t, N(t)$, scales as (cf. section 2.3)

$$
N(t) \sim r(t)^{d_{\mathrm{f}}} \quad\left(d_{\mathrm{s}} \leqslant 2\right) .
$$

For $d_{\mathrm{s}}>2$ diffusion is no longer recurrent, and

$$
N(t) \sim r(t)^{d_{\mathrm{w}}} \quad\left(d_{\mathrm{s}}>2\right) .
$$

Generalizing the Zwanzig [266] formalism (section 5.1), one writes for anomalous diffusion [290]

$$
\frac{t}{r^{d_{\mathrm{w}}}} \approx \frac{1}{N(t)} \sum_{i=1}^{N(t)} \frac{1}{w_{i}} .
$$

The exponent $d_{\mathrm{w}}$ describes anomalous diffusion on the fractal in the absence of wells. For a power-law distribution of wells, and a transition probability $w \sim 1 / V$, one obtains

$$
\frac{t}{x^{d_{\mathrm{w}}}} \sim \int_{w_{\min }}^{1} \frac{1}{w} P(w) \mathrm{d} w \sim w_{\min }^{-\alpha},
$$

where

$$
w_{\min }=N(t)^{-1 /(1-\alpha)} .
$$

As usual, the scaling of $w_{\min }$ is derived with the aid of a uniform distribution. Thus the diffusion exponent is $[136,290]$

$$
d_{\mathrm{w}}(\alpha)= \begin{cases}d_{\mathrm{w}}+\frac{d_{\mathrm{f}} \alpha}{1-\alpha} & \left(d_{\mathrm{s}} \leqslant 2\right), \\ \frac{d_{\mathrm{w}}}{1-\alpha} & \left(d_{\mathrm{s}}>2\right) .\end{cases}
$$


The result for $d_{\mathrm{s}}<2$ has been verified numerically on the Sierpinski gasket [136] and on percolation clusters [290]. Note that for one-dimensional systems (5.43) reduces to $(5.3 \mathrm{~b})$. Note also that for $d_{\mathrm{s}}>2$ we recover the result obtained using a continuous-time random-walk model [244].

\subsection{Anomalous diffusion on combs and fractals}

A problem related to diffusion on a lattice with a distribution of transition rates is diffusion on combs. Consider a comb in which the length of each tooth is distributed according to

$$
\varphi(L)=\gamma L^{-(1+\gamma)}, \quad \gamma>0, \quad L \geqslant 1 .
$$

This distribution leads to anomalous diffusion, whose characteristic exponents depend on the value of $\gamma$. When the average tooth length in finite $(\gamma>1)$ diffusion is regular, i.e. $\left\langle x^{2}\right\rangle \sim t$. Two techniques have been used [291] to calculate the diffusion exponents. The first approach uses a formalism involving the continuoustime random-walk (CTRW), and the second is a self-consistent scaling theory. The results of both analyses are in agreement, and are confirmed by numerical simulations.

The CTRW approach assumes a random walk without memory. That is to say, when the random walker returns to a site that has already been visited, he can see a tooth of a length $L$ different from that seen at the previous time. In this case the mean-square displacement along the backbone, $\left\langle x^{2}(n)\right\rangle$, can be expressed as

$$
\left\langle x^{2}(n)\right\rangle \equiv \sigma^{2}\langle j(n)\rangle,
$$

where $\sigma^{2}$ is the variance of the displacement of a single step of the random walk $\left(\sigma^{2}=1\right.$ for a symmetric nearest-neighbou $\mathrm{r}$ walk). The average is over all configurations of lengths, and $\langle j(b)\rangle$ is the expected number of steps that are taken along the backbone out of a total of $n$ steps [239]. The generating function of $\langle j(n)\rangle$ with respect to $n$ is denoted by $J(z)$ :

$$
J(z) \equiv \sum_{n}\langle j(n)\rangle z^{n} .
$$

Let $\psi_{n}$ be the probability that the interval between two successive sojourns into a tooth at a site of the backbone is equal to $n$, and let $\langle\Psi(z)\rangle$ be the corresponding generating function. It can be shown [239] that

Since [291]

$$
J(z)=\frac{\langle\Psi(z)\rangle}{(1-z)(1-\langle\Psi(z)\rangle)} .
$$

it follows that, as $z \rightarrow 1$,

$$
\left\langle\Psi_{n}\right\rangle \sim n^{(3+\gamma) / 2}
$$

$$
\langle\Psi(z)\rangle \sim 1-(1-z)^{(1+\gamma) / 2} .
$$

When this representation is substituted into (5.47) one finds that

$$
J(z) \sim(1-z)^{-(3+\gamma) / 2}
$$

Then, making use of a Tauberian theorem [292], one obtains

$$
\left\langle x^{2}(n)\right\rangle \sim n^{(1+\gamma) / 2},
$$


or

$$
d_{\mathrm{w}}= \begin{cases}4 /(1+\gamma) & (0<\gamma<1) \\ 2 & (\gamma \geqslant 1)\end{cases}
$$

The above analysis has the characteristics of a mean-field theory in that the spatial structure of the comb teeth is replaced by an average waiting-time distribution independent of the $x$-coordinate. Thus the detailed structure of the comb is taken into account in an approximate, rather than an exact, way. A self-consistent approach [291], that takes account of the detailed spatial structure in a more exact way is presented in the following. The effect of the teeth is to delay the walk along the backbone. The delays in a single tooth are characterized by an average waiting time $\tau(L)$ [260]:

$$
\tau(L) \sim L, \quad L \gg 1
$$

Thus a transition rate $w(L) \sim 1 / L$ is associated with a tooth of length $L$. Since the distribution of lengths has the asymptotic property shown in (5.44), it follows that the distribution of the waiting times is $p(w)$, where

$$
p(w)=\frac{\varphi(L)}{\mathrm{d} w / \mathrm{d} L} \sim w^{-(1-\gamma)} .
$$

The diffusion exponent $d_{\mathrm{w}}$ is calculated using (5.8).

$$
\frac{t}{\left\langle x^{2}\right\rangle} \sim \int_{w_{\min }}^{1} \frac{p(w)}{w} \mathrm{~d} w \sim w_{\min }^{\gamma-1}, \quad \gamma<1 .
$$

The detailed structure of the comb enters through the cut-off $w_{\min }$. This cut-off is a measure of the smallest transition rate the random walker sees during a displacement $x$. This minimum transition rate is obtained from the smallest of two characteristic lengths in the $y$-direction. One is the maximum tooth length within a distance $x$ along the backbone, which scales as $x^{1 / \gamma}$. The second is the span of a random walk diffusing in the $y$-direction when the teeth are infinitely long. This span $y$ is given by the relation

$$
y^{2} \sim t \sim x^{d_{\mathrm{w}}}
$$

The second of these relations reflects the anomalous diffusion along the backbone. The maximum span $L_{\max }$ is therefore the minimum of these two lengths:

$$
L_{\max }=\min \left(x^{d_{\mathrm{w}} / 2}, x^{1 / \gamma}\right) .
$$

Since $w \sim 1 / L, w_{\min } \sim 1 / L_{\max }$. It can be seen a posteriori that $x^{d_{\mathrm{w}} / 2}$ is smaller than $x^{1 / \gamma}$ for all $\gamma$. Substituting (5.57) into (5.55), one obtains

$$
t /\left\langle x^{2}\right\rangle \sim x^{-d_{w}(\gamma-1) / 2}
$$

from which one finds a self-consistent equation for $d_{\mathrm{w}}$ :

$$
d_{\mathrm{w}}=2-\frac{1}{2} d_{\mathrm{w}}(\gamma-1)
$$

and (5.52) follows.

The reason for the success of the 'mean-field' CTRW approach is that the very long teeth have a negligible effect, since (5.56) and (5.57) imply that a random walker rarely visits the tips of those teeth. Thus large fluctuations in the length of the teeth are not relevant, and the CTRW approach is valid. Equation (5.52) has been tested numerically [291] by performing random walks on random combs with several values 


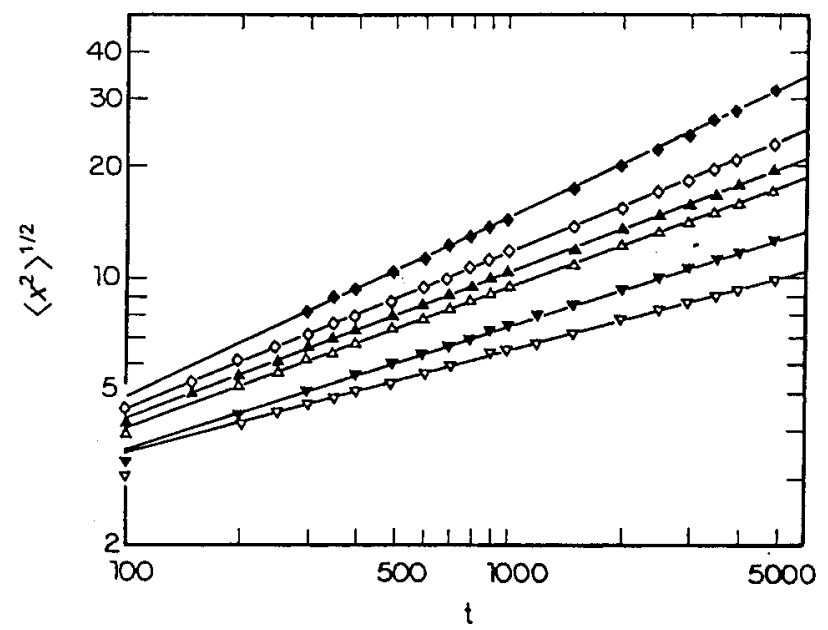

Figure 33. Plot of the mean-square displacement along the backbone, $\left\langle x^{2}\right\rangle^{1 / 2}$, as a function of $t$ for several values of $\gamma: \diamond, \gamma=1 ; \diamond, \frac{2}{3} ; \boldsymbol{\Delta}, \frac{1}{2} ; \Delta, \frac{2}{5} ; \nabla, \frac{1}{5} ; \nabla, \frac{1}{10}$.

of $\gamma$. Numerical results for $\left\langle x^{2}\right\rangle$ as a function of $t$ obtained using the exact enumeration method are shown in figure 33. For the values of $\gamma=1, \frac{2}{3}, \frac{1}{2}, \frac{2}{5}, \frac{1}{5}, \frac{1}{10}$ it was found from the slopes of figure 33 that $d_{\mathrm{w}}=2.08 \pm 0.1\left(d_{\mathrm{w}}=2\right.$ from $\left.(5.52)\right)$, $2.38 \pm 0.05$ (2.4), $2.69 \pm 0.05$ (2.67), $2.82 \pm 0.05$ (2.86), $3.24 \pm 0.10$ (3.33) and $3.60 \pm 0.10$ (3.63), in excellent agreement with the values predicted from (5.52).

A similar approach can be applied to calculate the long-time autocorrelation function $P_{0}(t) \sim t^{-d_{\mathrm{s}} / 2}$, which is the probability that a random walker initially at the origin is found at time $t$ at the origin. It was shown by Alexander and Orbach [45] that $d_{\mathrm{s}}=2 d_{\mathrm{f}} / d_{\mathrm{w}},(2.12)$. Since the random walker does not visit the tips of the longer teeth, $d_{\mathrm{f}}$ should represent the fractal dimension of the volume $M$ visited by the walker. This can be approximated as

$$
M \sim \sum_{L=1}^{x} L \sim x \int_{0}^{L_{\max }} L^{\prime} P\left(L^{\prime}\right) \mathrm{d} L^{\prime} \sim x L_{\max }^{1-\gamma},
$$

where $L_{\max }$ is found from (5.57). This leads to

$$
M \sim x^{d_{\mathrm{f}}} \equiv x^{1+d_{\mathrm{w}}(1-\gamma) / 2}
$$

or

$$
d_{\mathrm{f}}=1+\frac{1}{2} d_{\mathrm{w}}(1-\gamma) \text {. }
$$

This relation can also be derived by substituting $\tilde{\zeta}=1$ in the Einstein relation $d_{\mathrm{w}}=d_{\mathrm{f}}+\tilde{\zeta}$. Using (5.52) and (5.62), one obtains

$$
\frac{1}{2} d_{\mathrm{s}}=\left\{\begin{array}{cc}
\frac{1}{4}(3-\gamma) & (\gamma \leqslant 1) \\
\frac{1}{2} & (\gamma>1)
\end{array}\right.
$$

There are two limiting cases in which contact can be made with earlier results. When $\gamma>1$ the first moment of the length distribution in (5.44) is finite, and the mean sojourn time on a tooth is also finite. For the transition value $\gamma=1$ one finds $d_{\mathrm{w}}=2$ and $d_{\mathrm{s}}=\frac{1}{2}$, which is the same as for a one-dimensional random walk when the 
variance of the step length is finite. The case $\gamma=0$ corresponds to a comb in which the teeth are all infinite, for which it has been shown [239] that $d_{\mathrm{w}}=4$ and $d_{\mathrm{s}}=\frac{3}{2}$.

Note that, although $d_{\mathrm{s}}<2$ for all $\gamma$, the random walker visits only a subset of the comb that has a fractal dimension smaller than that of the comb, (5.62). This is in contrast with the situation for diffusion on isotropic fractals, for which a random walk is recurrent whenever $d_{\mathrm{s}}<2$. Indeed, for diffusion on finitely ramified loopless fractals (which are closely related to combs), diffusion is always recurrent. This is since for trees $d_{\mathrm{s}}=2 d_{l} /\left(1+d_{l}\right)$ (section 4.1 ), and $d_{\mathrm{s}}$ is always less than 2 .

It is interesting to see how the present approach can be used to obtain results for different fractal models. As an example, let us consider diffusion on the incipient infinite percolation cluster generated on a Cayley tree. This can be modelled by diffusion on the backbone of a comb. The excursions on dead ends are modelled by the excursions on the teeth of the comb. The distribution of dangling-end sites $s$ along the backbone is ostensibly [18]

$$
\varrho(s) \sim s^{-3 / 2} .
$$

It is physically reasonable to assume that the transition rates $\left\{w_{i}\right\}$ scale as the inverse of the dangling-end sizes, so that

$$
P(w)=\varrho(d) \frac{\mathrm{d} s}{\mathrm{~d} w} \sim w^{-1 / 2} .
$$

Using the results of section 5.2, one obtains $d_{\mathrm{w}}^{l}=3$, as has been found earlier (see, for example reference [57]).

\section{Biased diffusion in disordered media}

In this section we study the effect of an external bias field on the diffusion in disordered media. The field makes a diffusing particle more likely to move along the direction of the field than otherwise. In the case of a uniform non-random medium the effect of such a bias is to produce a drift velocity in the direction of the field that increases with the field. In the case of a disordered structure the bias has two competing effects. On the one hand, the bias pushes the walker along the direction of the field. On the other hand, dead ends create temporary traps, and in order to escape from them the walker must move against the field. Examples of physical realizations of the above situation are the cases of diffusion of particles in gels under gravity, or in centrifugal forces as in chromatographic columns [293], and hopping electron conduction in doped semiconductors in the presence of strong electric fields [294-296]. Several cases of bias diffusion on random structures have recently been studied extensively [240-242, 246, 259, 260, 276, 297-305]. The bias field, $E$ is modelled by giving the random walker a higher probability $P_{+}$of moving along the direction of the field, and a lower probability $P_{-}$of moving against it,

$$
P_{ \pm}=A(1 \pm E)
$$

where $A$ is an appropriate normalization factor. The motion of the walker in a transverse direction to the field is not affected. The field can be uniform, as the electric field between the plates of a condensor (Cartesian bias), or directed in topological space, as in a bias of hydrodynamic origin (topological bias). The hydrodynamic flow biases the particles along the direction of the flow, and the bias depends on the flow velocity. De Arcangelis et al. [304] studied the hydrodynamic 


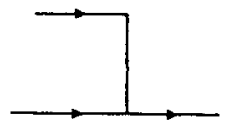

(a)

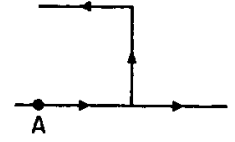

(b)

Figure 34. Comparison between (a) Cartesian bias with a field directed along the $x$-axis and (b) 'topological' bias for a simple structure. In $(b)$ fluid in injected at A, and every point in the flow experiences a field $E$ along the chemical distance from the origin A. The arrows denote the direction of the local bias in each bond.

transport using an iterative algorithm similar to the exact-enumeration method. Roux et al. [306] applied a transfer-matrix algorithm to a similar model. T. Ohtsuki and T. Keyes (1987, preprint) studied the problem using real-space renormalization. A distinction is made between the diffusive regime, in which a particle has a finite probability of diffusing against the direction of the current flow, and the convective regime, where backwards diffusion is not allowed. A much studied [259, 260, 276] simple case of hydrodynamic bias is the Knudsen limit. In this limit particles experience topological bias along the direction of the flow, but independent of the current velocity. More specifically, in the Knudsen limit, every bond experiences a constant bias field, which drives the walker away, in chemical distance, from a point source. The walker has an enhanced probability $P_{+}=A(1+E)$ that the next step increases the chemical distance from the source, and a decreased probability $P_{-}=A(1-E)$ that the next step decreases the chemical distance (figure 34). Diffusion with a constant bias has been studied on random structures such as selfavoiding walks [302], random combs [240, 260, 276, 299], and percolation clusters above criticality [259, 297-299, 301] and at criticality [246, 276, 298, 303].

It has been argued that for certain random structures a dynamical phase transition occurs as the bias field varies. For example [240, 241, 260], in a random comb with an exponential distribution of tooth lengths, a drift velocity that is finite below a critical bias $E_{\mathrm{c}}$ vanishes above $E_{\mathrm{c}}$. Moreover, above $E_{\mathrm{c}}$ the diffusion exponent $d_{\mathrm{w}}$ increases continuously with the magnitude of the bias [260]. There exist numerical data supporting a dynamical phase transition for bias diffusion on a random comb [260], and for a topological bias on percolation clusters [259] above $p_{\mathrm{c}}$. On the other hand, numerical data for Cartesian bias on percolation clusters [297] above $p_{\mathrm{c}}$, as well as on self-avoiding walks [302], are inconsistent with such a transition.

For biased diffusion on a random comb with a power-law distribution of tooth lengths, it has been shown theoretically that diffusion is anomalously slow, with $\left\langle r^{2}\right\rangle$ scaling as a power of $\ln t$, rather than of $t$. A similar anomalous logarithmic behaviour has been observed numerically in percolation clusters at criticality for Cartesian bias [303] and topological bias [276].

\subsection{Dynamical phase transition}

We shall consider biased diffusion on a random comb structure as shown in figure 35. This simple model encompasses the essential features of biased diffusion on disordered media, yet is simple enough to be treated rigorously. As we shall see in section 6.3, this model is particularly useful in the study of topological bias on percolation systems.

Consider a random comb with an exponential distribution of tooth lengths: 

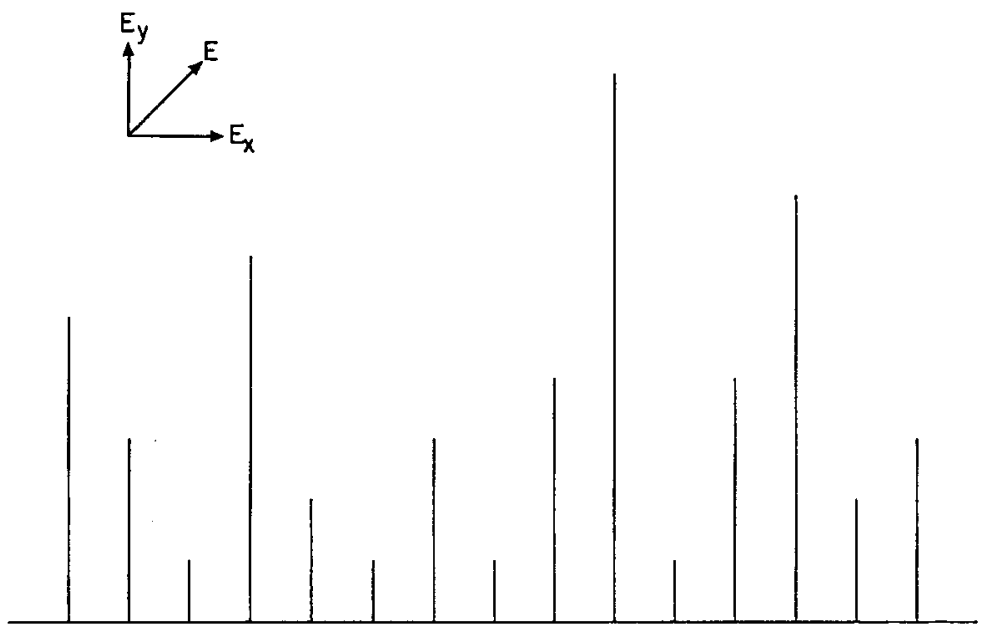

Figure 35. Bias diffusion on a random comb structure. The backbone is parallel to the $x$ axis. The bias field may in general have components along both the $x$ - and the $y$-directions.

$$
P_{0}(l)=\lambda^{l}|\ln \lambda|=\frac{1}{l_{0}} \exp \left(-\frac{l}{l_{0}}\right), \quad 0 \leqslant l \leqslant \infty,
$$

where $\lambda \equiv \exp \left(-1 / l_{0}\right)$. This choice is relevant to bias diffusion on percolation clusters above criticality. We first present the transport properties along the backbone of the comb when a field is applied along the $y$-direction. The physical effect of the teeth is to provide a set of random delays (or waiting times) along the backbone. A waiting time $\tau(l)$ is associated with a tooth of length $l$. To calculate $\tau(l)$, consider a random walker on a tooth of length $l$ at a distance $j(<l)$ from the backbone. Let $T_{j}$ be the mean first passage time to reach the backbone and let $\tau(l)$ be quantitatively taken as $\tau(l)=T_{1}$. Since the walker on the next step moves either to site $j+1$ (with probability $P_{+}$) or to site $j-1$ (with probability $P_{-}$), the following recursion relation can be written for $T_{j}[260]$ :

$$
T_{j}-1=\frac{1}{2}\left(1-E_{y}\right) T_{j-1}+\frac{1}{2}\left(1+E_{y}\right) T_{j+1} .
$$

Equation (6.3) is subject to the boundary conditions

$$
T_{j=0}=0, \quad T_{l}=T_{l-1}=\frac{2}{1-E_{y}},
$$

since $j=0$ and $j=l$ are 'absorbing' and 'reflecting' points respectively. The solution of (6.3) and (6.4) for $T_{1}$ gives

$$
\tau(l)=T_{1}=\frac{1}{E_{y}}\left[\left(\frac{1+E_{y}}{1-E_{y}}\right)^{l}-1\right] .
$$

Since the behaviour is dominated by the long teeth, one has in the asymptotic limit (see also reference [241]).

$$
\tau(l) \sim\left(\frac{1+E_{y}}{1-E_{y}}\right)^{l} \quad(l \gg 1) .
$$

The transition rate to pass by this tooth, $w(l)$, is given by the reciprocal of the mean waiting time $\tau(l)$ spent on it: 


$$
w(l) \sim\left(\frac{1-E_{y}}{1+E_{y}}\right)^{l} \equiv \exp \left(-\frac{l}{\Lambda}\right) .
$$

The rates $\{w(l)\}$ are random variables whose probability distribution $P(w)$ is directly related to the distribution $P_{0}(l)$ of the teeth by

$$
P(w)=P_{0}(l) \frac{\mathrm{d} l}{\mathrm{~d} w} .
$$

Substituting (6.2) and (6.7) into (6.8) yields

$$
P(w) \sim w^{-\alpha},
$$

where

$$
\alpha=1-\frac{\Lambda}{l_{0}}=1-\ln \lambda / \ln \left(\frac{1-E_{y}}{1+E_{y}}\right) .
$$

By this, the problem of biased diffusion on a random comb structure with exponential distribution of teeth is mapped to the problem of biased diffusion on a linear chain with a power-law distribution of transition rates. Using the results of sections 5.1 and 5.2, one obtains

$$
d_{\mathrm{w}}= \begin{cases}2 & (\alpha<0) \\ 2+\frac{\alpha}{1-\alpha} & (0 \leqslant \alpha \leqslant 1) .\end{cases}
$$

When the field has a non-zero component along the backbone ( $x$-direction) the results in section 5.1 for the case of unbiased diffusion are extended to bias diffusion. Because of the bias, the time $t$ that the walker takes to pass by a distance $x$ is proportional to the sum of the waiting times along the covered distance,

$$
t \sim \sum_{i=1}^{x} \tau_{i}=\sum_{i=1}^{x} \frac{1}{w_{i}}
$$

From $(6.9 a)$ and (6.11) one obtains

$$
t \sim x \int_{w_{\min }}^{1} \frac{1}{w} P(w) \mathrm{d} w \sim \begin{cases}x & (\alpha<0), \\ x w_{\min }^{-\alpha} & (\alpha>0) .\end{cases}
$$

For $\alpha \leqslant 0$ the ballistic result for biased diffusion on uniform Euclidean lattices, $t \sim x$, is recovered. The case $\alpha>0$ is more interesting. Since

we have $t \sim x^{d_{\mathrm{w}}}$, with

$$
w_{\min }=x^{-1 /(1-\alpha)},
$$

$$
d_{\mathrm{w}}= \begin{cases}1 & (\alpha<0), \\ 1+\frac{\alpha}{1-\alpha} & (0 \leqslant \alpha \leqslant 1) .\end{cases}
$$

The results for a general bias field can be summarized, using $(6.9 \mathrm{~b})$, as follows:

$$
d_{\mathrm{w}}= \begin{cases}d_{\mathrm{w}}^{0} & \left(E_{y}<E_{y}^{\mathrm{c}}(\lambda)\right), \\ d_{\mathrm{w}}^{0}+\frac{\ln \left[\left(1-E_{y}\right) /\left(1+E_{y}\right)\right]}{\ln \lambda} & \left(E_{y}>E_{y}^{\mathrm{c}}(\lambda)\right) .\end{cases}
$$




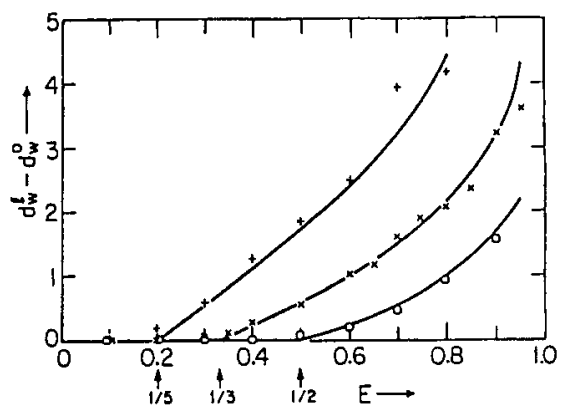

Figure 36. Dependence of $d_{\mathrm{w}}$ on the bias field $E_{y}$. Data are shown for the cases $\lambda=\frac{1}{3}(\bigcirc)$ and $\frac{1}{2}(\times)$ with $E_{x}>0$, and for $\lambda=\frac{2}{3}(+)$ with $E_{x}=0$. The solid curves represent the theoretical result of (6.15).

Here $d_{\mathrm{w}}^{0}$ is the diffusion exponent along the backbone in the absence of teeth:

$$
d_{\mathrm{w}}^{0}= \begin{cases}2 & \left(E_{x}=0\right) \\ 1 & \left(E_{x}>0\right)\end{cases}
$$

The critical bias field, $E_{y}^{\mathrm{c}}(\lambda)$ corresponding to $\alpha=0$ is given by [240, 241, 260]

$$
E_{y}^{\mathfrak{c}}(\lambda)=\frac{1-\lambda}{1+\lambda} .
$$

On crossing $E_{y}^{\mathrm{c}}(\lambda)$, there is a phase transition in the dynamics. For $E_{y}<E_{y}^{\mathrm{c}}$ the transport on the backbone is normal, but for $E_{y}>E_{y}^{\mathrm{c}}$ the field confines the walker to the teeth more effectively, with the result that $d_{\mathrm{w}}$ increases anomalously.

Equations (6.15) and (6.16) have been tested by performing extensive numerical studies of biased diffusion on random combs [260] using the exact-enumeration method [47, 83]. Results for the diffusion exponent $d_{\mathrm{w}}$ for a range of bias fields $E$ and for different values of $\lambda$ (characterizing the different distributions) are shown in figure 36 .

\subsection{Bias leading to logarithmic behaviour}

Consider a random comb with a power-law distribution of teeth [276]:

$$
P_{0}(l)=\gamma l^{-(1+\gamma)}, 1 \leqslant l \leqslant \infty .
$$

This choice is relevant to biased diffusion on percolation clusters at criticality. In this case the mass $l$ of the dangling ends emerging from the quasi-linear backbone is algebraically distributed (see section 6.3).

For a bias field along the $y$-direction the average time $\tau(l)$ that a random walker spends on a tooth of length $l$ is given by (6.6). Combining (6.6) and (6.17), one finds that the probability distribution $P(w)$ of transition rates along the backbone is given by

$$
P(w)=\frac{\gamma \Lambda}{w(\ln w)^{1+\gamma}} .
$$

This distribution for transition probabilities was discussed in section 5.2, and its solution was presented in (5.25) and (5.26). Making the trivial substitutions $V=l$ and $\beta=1 / \Lambda$, one obtains 


$$
\left\langle x^{2}\right\rangle \sim(\Lambda \ln t)^{2 \gamma}, \quad \Lambda=\left(\ln \frac{1+E_{y}}{1-E_{y}}\right)^{-1} .
$$

When a non-zero field is also applied along the $x$-direction, the time $t$ that the walker needs to pass by a distance $x$ on the backbone is obtained as in (6.10):

$$
t \sim \sum_{i=1}^{x} \tau_{i} \sim x \int_{w_{\min }}^{1} \frac{\mathrm{d} w}{w^{2}(\ln w)^{1+\gamma}} .
$$

Since

$$
w_{\min }=\exp \left(-\frac{x^{1 / \gamma}}{\Lambda}\right)
$$

one obtains from $(6.20)$

$$
\left\langle x^{2}\right\rangle \sim(\Lambda \ln t)^{2 \gamma}
$$

Hence for any direction of the field in the $(x, y)$ plane the motion of the walker is characterized by the same logarithmic time dependence.

Computer simulations of random walks on random combs with various values of $\gamma$ and $E$ have been performed [276] in order to test (6.22). Representative results for $\gamma=2$, and several values of $E$, are shown in figure 37 . The results are in good agreement with (6.19) and (6.22).

The above results are very slightly affected for comb-like structures with backbones of a higher dimension. In fact, for a bias field with a non-zero component along the backbone ( $x$-direction), the results do not change at all. For a bias field perpendicular to the backbone, the results change only quantitatively. For example, for a power law distribution of teeth, emerging from a $d$-dimensional backbone, the mean-square displacement is

$$
\left\langle r^{2}\right\rangle \sim \begin{cases}{[\Lambda(E) \ln t]^{2 \gamma}} & (d=1) \\ {[\Lambda(E) \ln t]^{\gamma}} & (d \geqslant 2)\end{cases}
$$

\subsection{Biased diffusion on percolation clusters}

The problem of non-biased diffusion on percolation clusters has been described in detail in section 3. It is well established that the r.m.s. displacement as a function of time is described adequately by a power law,

$$
\left\langle r^{2}\right\rangle \sim t^{2 / d_{\mathrm{w}}} \text {. }
$$

The question of what happens when a uniform (Cartesian) bias field is applied is controversial. Barma and Dhar [240] have predicted that above the percolation threshold the drift velocity vanishes above a critical field. On the other hand, Bottger and Bryskin [294] and also Pandey [297] on the basis of numerical simulations of percolation in $d=3$, suggest that the drift velocity goes to zero only for an infinite bias field (corresponding to $E=1$ ), and that there is no dynamical phase transition.

The problem of biased diffusion on percolation may be modelled by biased diffusion on random combs. The motion of the walker along the backbone of the percolation cluster is analogous to the motion along the backbone of the comb. Frequently, the walker sojourns at a dangling end of the cluster, spending there a typical time characteristic of the size of the dangling end. This is similar to the time 
(a)

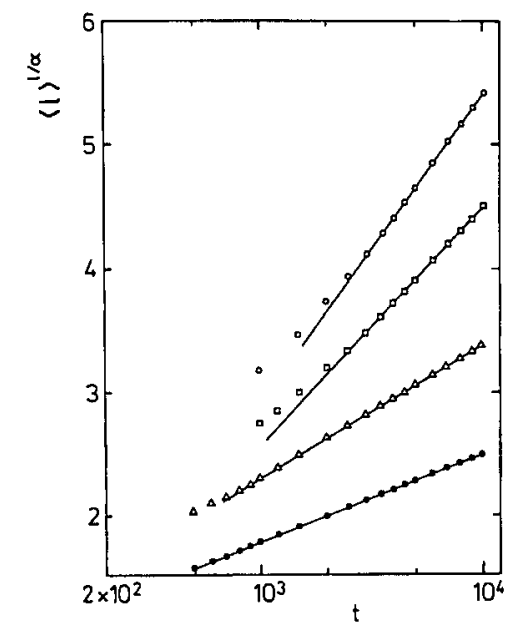

(b)

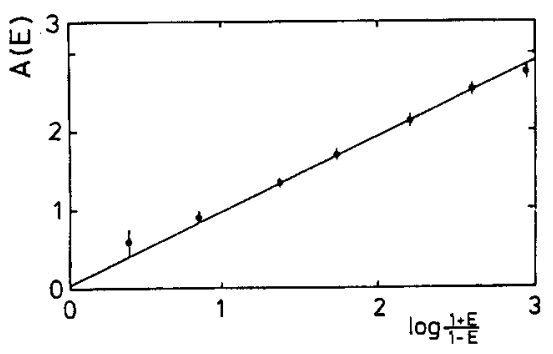

Figure 37. (a) Plot of $\langle x\rangle^{1 / \gamma}$ against $\ln t$ for diffusion on the random comb with $\gamma=2$ for different values of bias fields: $\bigcirc, E=0.9 ; \triangle, 0.7 ; \square, 0.4 ; \bigcirc, 0.2$. The curves represent the best asymptotic linear fits. (b) Dependence of $A\left(E_{y}\right)$ on $E_{y}$. The points represent the inverse of the slopes presented in $(a)$, and the curve is plotted from (6.19). For the simulations, combs of size $L=300$ and averages over 100 configurations each were taken.

spent by the walker in the teeth of the comb. Although for $d<6$ the backbone is not really a one-dimensional object, the analogy is not spoiled, because of the insensitivity of transport properties to the dimension of the backbone (as discussed at the end of section 6.2). For $p>p_{\mathrm{c}}$ the mass of dangling ends is, ostensibly, exponentially distributed, and for $p=p_{\mathrm{c}}$ it follows a power-law distribution. The corresponding distribution for the length of the teeth in the random comb is chosen accordingly. Much of the research [240, 241, 294, 297-303] on biased diffusion deals with a uniform (Cartesian) bias field. In this case, not only dead ends but also backbends [307] act as temporary traps. This effect can be modelled, similarly to the trapping in dead ends, by trapping in the teeth of the random comb.

In a recent work [259] diffusion on the infinite percolation cluster above the percolation threshold, $p>p_{\mathrm{c}}$, under the influence of a bias field $E$ in topological space ('topological bias') has been studied. Numerical simulations using the exact-enumeration method support the dynamical phase transition $[260,298]$ of section 6.1 . The mean chemical distance $\langle l\rangle$ travelled by a random walker can be writen as [57]

$$
\langle l\rangle \sim t^{1 / d_{\mathrm{w}}^{l}}
$$


where $d_{\mathrm{w}}^{l}$ is the topological diffusion exponent. The numerical data [259] suggest that below a critical field $E_{\mathrm{c}}(p)$ a velocity drift in the chemical space exists, reflected by $d_{\mathrm{w}}^{l}=1$. Above $E_{\mathrm{c}}(p)$ diffusion is anomalous and non-universal: the diffusion exponent $d_{\mathrm{w}}^{l}$ increases with $E$ as

$$
d_{\mathrm{w}}^{l}=A(p) \ln \left(\frac{1+E}{1-E}\right) \quad\left(E>E_{\mathrm{c}}(p)\right) .
$$

Here $A(p)$ is analogous to $(\ln \lambda)^{-1}$ in $(6.15 a)$. It is a characteristic of the distribution of dangling ends, and it decreases monotonically with the concentration $p$. The theoretical derivation of (6.26) depends crucially on the assumption that the effective dead-end's length in percolation clusters follow an exponential distribution. The numerical results [259] supporting (6.26) indirectly confirm this assumption.

Studies of bias diffusion on percolation systems at the percolation threshold $p_{\mathrm{c}}$ were carried out by several authors [246, 276, 297, 298, 303]. Ohtsuki and Keyes [246] applied a real-space renormalization-group method and found for Cartesian bias $\langle r\rangle \sim t^{x}$ with $x<1$. Preliminary numerical data for Cartesian-bias diffusion in $d=3$ percolation at $p_{\mathrm{c}}$ presented by Pandey [297] suggest that either $\left\langle r^{2}\right\rangle \sim t^{k}$ with very small $k$, or else $\left\langle r^{2}\right\rangle \sim(\ln t)^{x}$. Later numerical results for $d=2$ and 3 , presented by Stauffer [303], support the logarithmic time dependence. In a recent work [276], topological bias diffusion on the infinite incipient percolation cluster at criticality in $d=2$ and on a Cayley tree, was studied theoretically and numerically. An

(a)
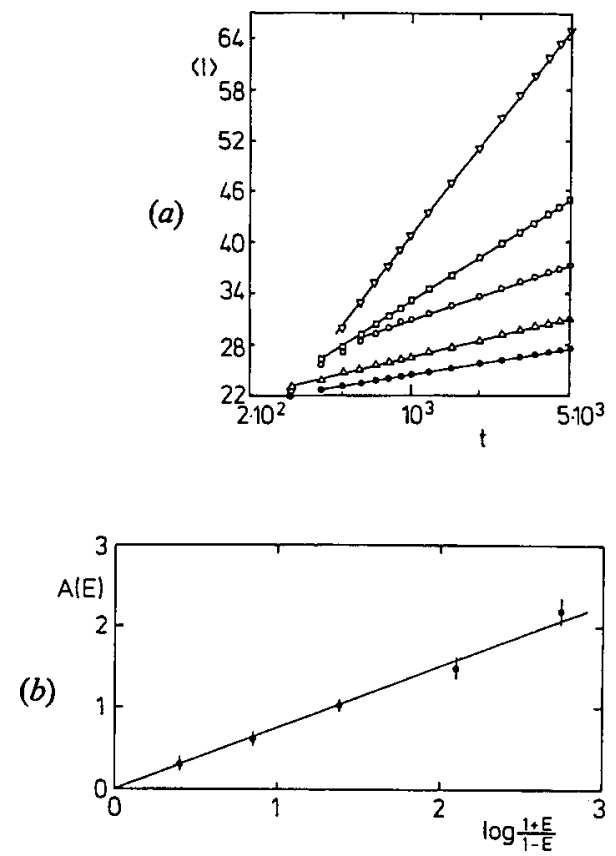

Figure 38. (a) Plot of $\langle l\rangle$ against $\ln t$ on percolation clusters generated in $d=2$ at criticality, for different values of bias fields: $\bigcirc, E=0.9 ; \triangle, 0.8 ; \bigcirc, 0.7 ; \square, 0.5 ; \nabla, 0.3$. The solid lines are best linear fits. (b) Dependence of $A(E)$ on $E$. The points represent the inverse of the slopes presented in $(a)$, and the line represents the theory (6.19). For the calculations, clusters of up to 200 shells were generated and averages were made over 100 configurations. 
(a)

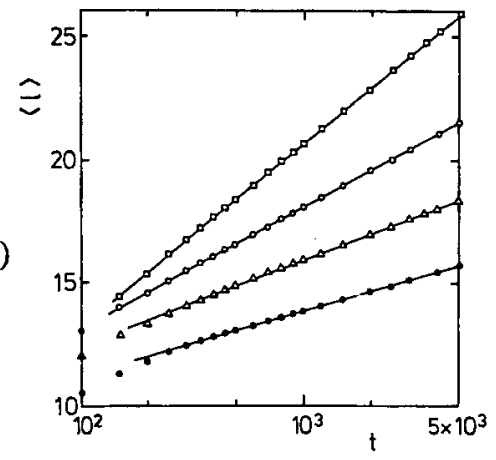

(b)

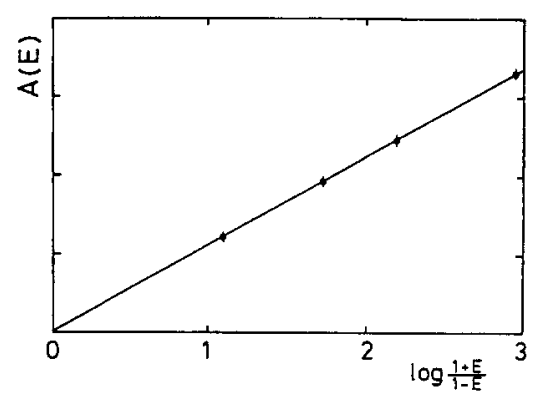

Figure 39. (a) Plot of $\langle l\rangle$ against $\ln t$ on percolation clusters generated on a Cayley tree at criticality, for different values of bias fields: $\bigcirc, E=0.9 ; \triangle, 0.8 ; \bigcirc, 0.7 ; \square, 0.5$. The solid lines are best linear fits. (b) Dependence of $A(E)$ on $E$. The points represent the inverse of the slopes presented in $(a)$, and the curve is plotted from (6.19). For the simulations, clusters of up to 200 shells and averages over 100 configurations were taken.

assumption of a power-law distribution for the effective dangling-end lengths in percolation clusters leads (see section 6.2) to a logarithmic time dependence:

$$
\langle l\rangle \sim(\Lambda(E) \ln t)^{\gamma}
$$

where $\gamma$ characterize s the distribution of dangling ends and $\Lambda(E)$ is given by (6.7). To test the validity of (6.27) for percolation clusters, computer simulations of random walks with various values of topological bias fields were performed using the exactenumeration method. Results for $d=2$ percolation clusters and percolation on a Cayley tree are shown in figures 38 and 39. For both cases the best agreement with (6.27) is obtained for $\gamma \approx 1$.

The value of $\gamma \approx 1$ for percolation on a Cayley tree (mean field) is explained by the following scaling argument. A random walker along the backbone of the incipient infinite cluster is temporarily trapped along its way by dangling ends. It is plausible that the mass $s$ of the dangling ends is distributed the same as the mass of clusters [18]

$$
P_{0}(S) \sim S^{-\tau+1}, \quad \tau=\frac{5}{2}
$$

The path length $l$ is related [57] to the mass $S$ by $S \sim l^{2}$. Hence the distribution of the path lengths is

$$
P(l)=P_{0}(S) \frac{\mathrm{d} S}{\mathrm{~d} l} \sim l^{2(1-\tau)+1}=l^{-2} .
$$


Comparing (6.29) and (6.17), we see that $\gamma=1$ for percolation on the Cayley tree. For percolation in $d<6$ the above argument does not apply. For example, in $d=2$ one obtains $\gamma \approx 0.1$ which is much smaller than the measured numerical value, $\gamma \approx 1.0$. This is since the structure of the dangling ends is complex and contains loops. An effective $\gamma$ is needed and cannot be calculated simply from $\tau$.

\subsection{Bias along the backbone}

For the case of a bias induced by the hydrodynamic flow in porous media [29, 308, 309], it is relevant to study a model with a bias field along the backbone alone. This is since the net flow in the dangling ends is zero. In the following we review a simple realization of such a model.

Consider the case of biased diffusion along the backbone of the comb with a power-law distrubiton of dead ends (6.17). In this case the particle has a drift along the backbone $\left(E_{x} \neq 0\right)$ and diffuses regularly along the dead ends $\left(E_{y}=0\right)$. The average time a particle spends on a tooth of size $l$ is given by (6.5) in the limit $E_{y} \rightarrow 0$ :

$$
T_{i} \equiv w^{-1} \underset{E_{y} \rightarrow 0}{=} 2 l
$$

Using (6.8) and (6.17), one obtains

$$
P(w)=2^{\gamma} \gamma w^{-(1-\gamma)} .
$$

The diffusion exponent $d_{\mathrm{w}}$ is found in a self-consistent scaling approach, similar to that of section 5.5. The total time spent on the backbone when a particle moves a distance $x$ is

$$
t \sim \sum_{i=1}^{x} \frac{1}{w_{i}} \sim x \int_{w_{\min }}^{1} \frac{1}{w} P(w) \mathrm{d} w \sim \begin{cases}x & (\gamma>1) \\ x w_{\min }^{-(1-\gamma)} & (\gamma<1)\end{cases}
$$

The quantity $w_{\min }$ is a measure of an effective smallest transition probability that the random walker sees during the displacement $x$. This transition probability is the inverse of an effective maximum span $l_{\max }$ made by the walker in the $y$-direction. This $l_{\max }$ is the smaller of two lengths characterizing the walk along the $y$-direction. One is the maximum tooth length within a distance $x$ along the backbone, which scales as $x^{1 / \gamma}$. The second is the span of a random walk diffusing in the $y$-direction, assuming infinite teeth. This span $y$ is found from the relation

$$
y^{2} \sim t \sim x^{d_{\mathrm{w}}} .
$$

The first relation describes regular diffusion along a tooth. The second of these relations reflects the anomalous diffusion expected along the backbone because of the sojourns of the particles along the teeth. (The diffusion exponent $d_{\mathrm{w}}$ will be calculated self-consistently.) The effective maximum span along the $y$-direction is

$$
l_{\max } \sim \min \left(x^{d_{w} / 2}, x^{1 / \gamma}\right) .
$$

Following the same approach as in section (5.5), one finds

$$
d_{\mathrm{w}}= \begin{cases}1 & (\gamma>1) \\ 2(1+\gamma)^{-1} & (0<\gamma<1) .\end{cases}
$$


It is evident that the diffusion along the teeth slows the transport along the backbone (compare to (5.52)) in spite of being regular. This slowing down is characterized by a diffusion exponent $d_{\mathrm{w}}>1$.

Similar arguments can be applied for the case of percolation systems in high dimensions $(d \geqslant 6)$. To this purpose, consider diffusion on the percolation cluster generated on a Cayley tree. The bias field acts on the particle only along the backbone of the tree, and the motion is diffusive in the dangling ends. The analogy between percolation on the Cayley tree and random comb with $\gamma=1$ used in section 6.2 cannot be used simple-mindedly here (substitution of $\gamma=1$ in (6.35) yields an incorrect result). The diffusion on the dangling ends of the percolation cluster is unbiased, but is anomalous $\left(d_{\mathrm{w}}^{l}=3\right)$, in contrast with diffusion on the teeth of the comb, which is normal with $d_{\mathrm{w}}^{l}=2$. The distribution of the mass of dangling ends, $S$, along the backbone is given by (6.28): $P_{0}(S) \sim S^{-\tau+1}$.

Similarly to the result in (6.30), the transition probabilities $\left\{w_{i}\right\}$ along the backbone scale as the inverse of the corresponding dangling-end mass:

$$
w \sim \frac{1}{S} .
$$

Thus the distribution of these transition probabilities for $w$ is

$$
P(w)=P_{0}(S) \frac{\mathrm{d} S}{\mathrm{~d} w} \sim w^{-(3-\tau)} .
$$

Let $l_{\|}(t)$ and $l_{\perp}(t)$ be the chemical distances traversed by the walker in $t$ steps on the backbone and on the dead ends respectively. Similarly to (6.33), $l_{\perp}$ and $l_{\|}$scales as

$$
l_{\perp}^{d_{\mathrm{w}}^{l}(E=0)} \sim t \sim l_{\|}^{d_{\mathrm{w}}^{l}} .
$$

The first relation results from the fact that no field is acting along the dangling ends (unbiased diffusion), and the second relation described the bias diffusion along the backbone. Since $S \sim l_{\perp}^{d_{l}}$, using (6.38) we obtain

$$
S \sim l_{\|}^{d_{\mathrm{w}}^{l} d_{l} / d_{\mathrm{w}}^{l}(0)} .
$$

The time spend on the backbone when a particle moves a distance $l_{\|}$is

$$
t \sim \sum_{i=1}^{l_{\|}} \frac{1}{w_{i}}=l_{\|} \frac{1}{w} P(w) \mathrm{d} w \sim l_{\|} w_{\min }^{-(3-\tau)}, \quad \tau<3 .
$$

Following the approach of section 5.5, one obtains

$$
d_{\mathrm{w}}^{l}=\frac{1}{1-(3-\tau) d_{l} / d_{\mathrm{w}}^{l}(0)} .
$$

Substituting the known exponents for percolation on the Cayley tree, $d_{\mathrm{w}}^{l}(0)=3$, $\tau=\frac{5}{2}, d_{l}=2$, one obtains

$$
d_{\mathrm{w}}^{l}=\frac{3}{2} .
$$

It would be interesting to confirm this result by an independent theoretical approach, and to test it numerically. For low dimensions, $d<6,(6.41)$ is not expected to yield good results, since the backbone is not exactly linear as assumed in (6.40). 


\subsection{Time-dependent fields}

Transport properties of disordered systems under the influence of time dependent fields have attracted considerable interest [37, 48, 200, 310-312]. Indeed, the case of an alternating bias field of small amplitude (the linear response regime) is closely related to the dynamical (a.c.) conductivity, and has been studied both experimentally [37, 311] and theoretically [37, 48, 200, 310].

Following the approach of Gefen et al. [48], the frequency dependence of the a.c. conductivity $\sigma(\omega)$ can be analysed using the scaling form

$$
\sigma(\omega)=\xi^{-\tilde{\mu}} s(\omega \tau), \quad \tau=\xi^{d_{\mathrm{w}}} .
$$

The factor $\xi^{-\tilde{\mu}}$ follows from the d.c. limit $\omega \rightarrow 0$, and $\tau$ is a typical time scale of the system derived from the anomalous diffusion. In the limit $\omega \tau \gg 1$ linear response theory applies, and $\sigma(\omega)$ is independent of $\xi$; therefore

$$
\sigma(\omega) \sim \omega^{\tilde{\mu} / d_{\mathrm{w}}} \quad(\omega \tau \gg 1) .
$$

Using the Kramers-Kronig relation for the dynamical dielectric constant $\epsilon(\omega)$,

$$
\epsilon(\omega)-1 \sim P V \int \frac{\mathrm{d} \omega^{\prime} \sigma\left(\omega^{\prime}\right)}{\omega^{\prime}\left(\omega^{\prime}-\omega\right)},
$$

one obtains

$$
\epsilon(\omega)=\omega^{2-\beta / \nu} f(\omega \tau)
$$

In the linear response regime, $\tau \gg 1$,

$$
\epsilon(\omega) \sim \omega^{-1+\tilde{\mu} / d_{\mathrm{w}}} .
$$

An alternative approach [310] to obtain the frequency dependence is by using the result of Scher and Lax [286] valid for the linear response regime, relating $\sigma(\omega)$ to the mean-square displacement $\left\langle R^{2}(t)\right\rangle$ :

$$
\sigma(\omega) \sim n \omega^{2} \lim _{\eta \rightarrow 0} \int_{0}^{\infty} \exp (-\mathrm{i} \omega t) \exp (-\eta t)\left\langle R^{2}(t)\right\rangle \mathrm{d} t .
$$

Here $n$ is the charge density, which scales as $n \sim \xi^{d_{\mathrm{f}}-d} \sim \omega^{\left(d-d_{\mathrm{f}}\right) / d_{\mathrm{w}}}=\omega^{\beta / \nu d_{\mathrm{w}}}$. From (6.57) it follows that

$$
\sigma(\omega) \sim \exp \left[-i\left(1-\frac{2-\beta / \nu}{d_{\mathrm{w}}}\right) \frac{\pi}{2}\right] \omega^{1-(2-\beta / \nu) d_{\mathrm{w}}} .
$$

Since $d_{\mathrm{w}}=2+\tilde{\mu}-\beta / \nu$, (3.18), this is exactly the same result as in (6.44), and in addition there is also a prediction for the phase shift between the current and field.

Bernasconi et al. [37] successfully explained the anomalous frequency dependence of the conductivity observed in the one-dimensional superionic conductor hollandite. They used a model with an exponential distribution of potential barriers, and a Boltzmann distribution of transition rates. It predicts an anomalous low-frequency conductivity, $\sigma(\omega) \sim(-\mathrm{i} \omega)^{\nu}$, with $\nu=\left(1-T / T_{\mathrm{c}}\right) /\left(1+T / T_{\mathrm{c}}\right)$ for $T<T_{\mathrm{c}}$, and a mobility transition for $T=T_{\mathrm{c}}$. This result is consistent with the result of (5.37) in section 5.3, which was derived for diffusion in the same model. The equivalence with (5.37) is evident when considering the relation $\sigma(\omega) \sim(-\mathrm{i} \omega)^{1-2 / d_{\mathrm{w}}}$, obtained from (6.49) (for a one-dimensional system $\beta=0$ ). However, the theory for $\sigma(\omega)$ and $\epsilon(\omega)$, discussed above, has been found [311] to be less successful in explaining the results for $d=2$ percolation. Laibowitz and Gefen [311] measured $\sigma(\omega)$ and $\epsilon(\omega)$ for thin gold films near the percolation threshold. They found that $\sigma(\omega) / \epsilon(\omega) \sim \omega$, in 


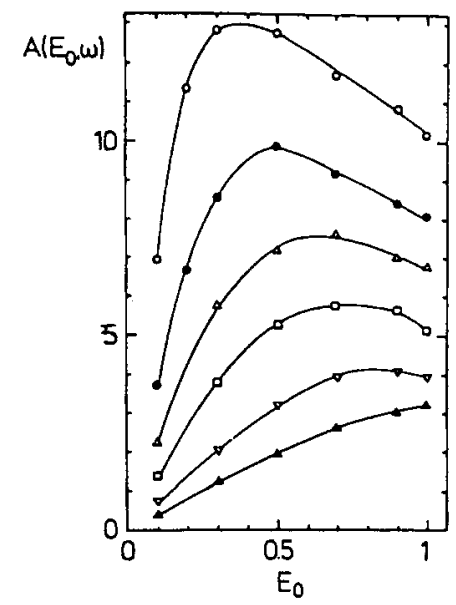

Figure 40. Plot of $A\left(E_{0}, \omega\right)$ as a function of $E_{0}$ for various values of frequency $\omega$ : $\bigcirc$, $\omega=0.002$;

$\boldsymbol{O}, 0.005 ; \triangle, 0.01 ; \square, 0.02 ; \nabla, 0.05 ; \boldsymbol{\Delta}, 0.1$.

accordance with (6.44) and (6.47), but that $\sigma(\omega) \sim \omega^{0.95}$, which strongly disagrees with the prediction of $(6.44)$ that $\sigma(\omega) \sim \omega^{\mu / d_{\mathrm{w}}}=\omega^{0.34}$. This discrepancy has been attributed [311] to the neglect of electron-electron interactions.

So far, only the linear response regime has been discussed. Harder et al. [310] studied the nonlinear response in two-dimensional percolation clusters. They found that for large applied fields the phase shift is still consistent with the shift predicted by linear response theory, but that the amplitude of the response is markedly different, and shows strong nonlinear effects. In figure 40 the amplitude of the displacement of a particle, $A\left(E_{0}, \omega\right)$, under the influence of a bias field $E=E_{0} \sin \omega t$ is shown. For low frequencies there is a strong nonlinearity.

\section{Trapping and diffusion-limited reactions}

The range of applications of the theory of diffusion in disordered media is immense. Among the most important are the kinetics of trapping processes, and diffusion-controlled reactions. Practical applications include various dynamical processes occurring in (fractal) disordered media such as powders, porous earth and rocks [313], amorphous solids [314], atmospheric dust, micellar systems [315, 316], and polymers in solution [317]. Physical examples include electron-hole recombination in random surfaces and in amorphous solids, exciton trapping and annihilation [251, 318], luminiscence, and slow drug release from a leaky matrix [319], to name just a few. Whenever the trapping process (for example of an exciton) or the reaction (for example between particles) is rapid in comparison with the characteristic time scale associated with the diffusion mechanism of motion of these excitons, or particles, the diffusion process becomes the most important factor in determining the kinetics of the system. Such systems are said to undergo a 'diffusionlimited', or a 'diffusion-controlled' process, and are the subject of this section.

The simplest cases of trapping (whether perfect or temporary) or a random walker, of one-species annihilation, $\mathrm{A}+\mathrm{A} \rightarrow$ inert, and two-species annihilation, $\mathrm{A}+\mathrm{B} \rightarrow$ inert, already contain the basic ingredients giving rise to the characteristic 
anomalous dynamics of diffusion-limited processes in disordered media. Most of the research work has been carried out for just such systems, and we review it below.

\subsection{Trapping}

The problem of trapping is fundamental for the understanding of kinetics of reactions. Image a random walker in a medium that is filled with static trapping sites at a finite concentration. The random walker represents some kind of excitation, or an active chemical species, and the trapping centres could be sites at which the excitation is dissipated, being converted to some other kind of energy, or they could represent some other chemical species that combines with the first, thus deactivating it. The question of interest is: what is the trapping rate, or, equivalently, the survival probability, or how the concentration of random walkers decays with time. We assume that trapping occurs immediately upon the encounter of a random walker with a trapping centre, so that the process is diffusion-limited.

For traps randomly distributed in a $d$-dimensional Euclidean space, the asymptotic result for the decay of $n(t)$, the number of random walkers, is [126, 127]

$$
n(t) \sim \exp \left\{-a c^{2 /(d+2)} t^{d /(d+2)}\right\} .
$$

Here $c$ is the concetration of traps and $a$ is a dimension-dependent constant. This result has been rigorously proved [126] for $t \rightarrow \infty$. For the one-dimensional case several authors [131, 320, 321] have presented a more detailed result, which also includes the prefactor to the exponential dependence. Weiss and Havlin [311] prove that in $d=1$ the diffusion of the surviving particles is anomalous, with $\left\langle R^{2}(t)\right\rangle \sim t^{2 / 3}$. Equation (7.1) is best understood, as shown by Grassberger and Procaccia [127], as being the consequence of the very large, but rare, trap-free regions, enhancing the survival probability of walkers in the long-time limit. Indeed, the probability of having a trap-free region of volume $V$, is according to the Poisson distribution,

$$
P_{0}(V)=\exp (-c V) .
$$

The probability $P(V, t)$ that a given random walker survives for a time $t$ in a trapfree region of volume $V$ enclosed by a trapping boundary is, asymptotically,

$$
P(V, t) \sim \exp \left(- \text { const } \times \frac{t}{V^{2 / d}}\right) .
$$

The survival probability $S(t)$, and hence the mean number of walkers $n(t)$, is dominated by

$$
S(t) \sim n(t) \sim \max _{\{V\}}\left[P_{0}(V) P(V, t)\right],
$$

giving rise to the result (7.1).

The above results easily generalize to trapping in fractal space. The only difference is that, because of anomalous diffusion, the scaling of time with the volume covered by the walker is $t \sim V^{d_{\mathrm{w}} / d_{\mathrm{f}}}=V^{2 / d_{\mathrm{s}}}$; hence, instead of (7.3), one has

$$
P(V, t) \sim \exp \left(\text { const } \times \frac{t}{V^{2 / d_{\mathrm{s}}}}\right) .
$$

On using this result and (7.4), one finally arrives at [322, 323]

$$
n(t) \sim \exp \left\{\text { const } \times c^{2 /\left(d_{\mathrm{s}}+2\right)} t^{d_{\mathrm{s}} /\left(d_{\mathrm{s}}+2\right)}\right\} .
$$



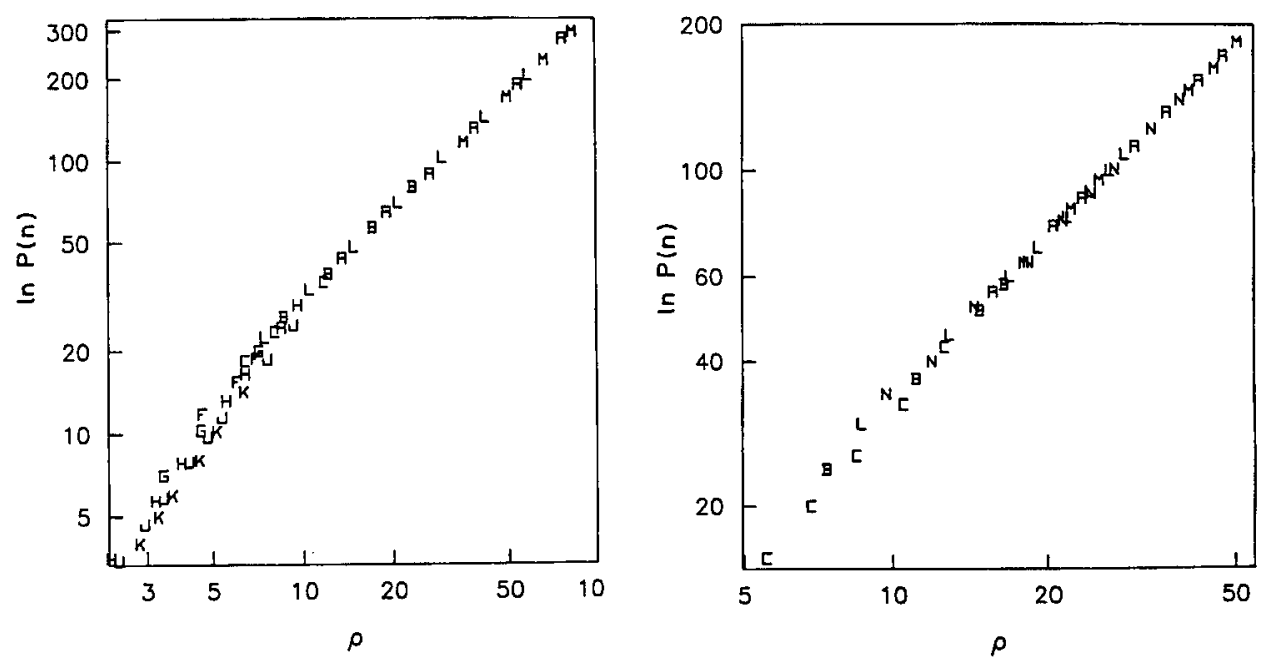

Figure 41. (a) Results for $\ln P(n)$ for different values of trap concentrations $c$ in $d=2$ dimensions and step sizes $n$, plotted as a function of $\varrho=[-\ln (1-c)]^{1 / 2} n^{1 / 2}$. (b) Results for $\ln P(n)$ for different values of trap concentrations $c$ in $d=3$ dimensions and step sizes $n$, plotted as a function of $\varrho=[-\ln (1-c)]^{2 / 5} n^{3 / 5}$. The results shown here have been obtained by the exact-enumeration method. (After Havlin et al. [130].)

Thus (7.6) is exactly the same as (7.1), except that $d$ is replaced by $d_{\mathrm{s}}$.

The question of the range of validity of (7.6) or (7.1) has recently attracted much interest [130, 323, 324]. Havlin et al. [130] found that for two and three dimensions the asymptotic behaviour of $n(t),(7.1)$ can be observed when the survival probability drops below $10^{-13}$ (figure 41). These results are based on exact-enumeration simulation methods. In spite of the extreme conditions required for the onset of asymptotic behaviour, it has been observed numerically in Monte Carlo simulations for trapping in $d=1[129,325]$, in percolation clusters in two and three dimensions [322]. Very recently it has been observed experimentally [326] by monitoring the electron-transfer reaction from methyl viologen monocations to colloidal platinum in an aqueous solution. A decay is reported there of the form $n(t) \sim \exp \left(-\alpha t-\beta t^{d /(d+2)}\right) \quad$ (in $d=3$ ), which is in agreement with the scaling prediction of Redner and Kang [327] for the case of mobile traps. For the trapping of particles in percolation clusters, Shapir [326] has found that the long-time behaviour is dominated by the more ramified clusters, with $d_{\mathrm{s}}=1$, leading to onedimensional behaviour in any dimension. No numerical simulations or experimental observations of this phenomenon are currently available.

For the important regime of short time $[133,328]$ the cumulant expansion

$$
S(t)=\exp \left\{\sum_{j} k_{j, t}[-\ln (1-c)]^{j} / j !\right\}
$$

can be truncated after a few terms, and it completely describes the trapping process. Here $k_{j, t}$ are cumulants of $S(t)$, the number of distinct sites visited by a randomwalker after $t$-steps. Zumofen et al. [133] combined the results for the short- and the large-time regimes into a scaling form

$$
S(t) \sim \exp [-f(x)], \quad x \equiv \ln (1-c) t^{d_{s} / 2}
$$


and

$$
f(x)= \begin{cases}\sum_{j} a_{j}(-x)^{j} / j ! & \text { for small } x \\ A x^{2 /\left(d_{\mathrm{s}}+2\right)} & \text { for large } x .\end{cases}
$$

where the $a_{j}$ and $A$ are constants. This scaling form works nicely for small $x$, reasonably well for the Sierpinski gasket and in $d=1$, but yields rather poor results for the square lattice. Obviously, a full answer to the problem of trapping that includes all the different regimes is still required.

We note that the case of imperfect trapping, in which a trap captures the random walker with a probability $f<1$, is also of interest. The particular case of temporary traps has been extensively discussed in section 5. Weiss and Havlin [329] generalized the imperfect-trapping problem to the non-Markovian case where a trap is characterized by a set of probabilities $\left\{f_{j}\right\}$. Here $f_{j}$ is the probability that the $j$ th encounter leads to a trapping event. They show that for such traps, when distributed on a line $(d=1)$, the asymptotic survival probability of a walker falls off as $t^{1 / 2} \exp \left(-a t^{1 / 3}\right)(\mathrm{cf} .(7.1))$, provided that the $f_{j}$ have an associated finite first moment. However, when $f_{j}$ is asymptotically proportional to $1 / j^{1+\alpha}$, where $0<\alpha<1$, the survival probability falls off as $1 / t^{\alpha}$. Another problem of interest is that of the trapping of random walkers that interact amount themselves. The particular case of a hard-core interaction between the diffusing particles is discussed and reviewed in section 8.1 .

\subsection{One-species annihilation and the effect of diffusion}

While trapping is a good model for the dissipation of excitations in disordered media, it is insufficient as a model for chemical reactions. This is due to the assumption made in the trapping model that the traps are static. In chemical reactions all the reactions are in motion, giving rise to different kinetics than predicted by the trapping model.

The dramatic effect of diffusion in a diffusion-controlled process, as opposed to a reaction-limited process (described by the mean-field rate equations), can already be seen in the simple case of one-species annihilation [128, 330-335]. Imagine a system containing particles of just one species A. The particles diffuse, and, on collision of two particles, either one is annihilated or they combine to form an inert species that does not participate in the process. The two cases are represented schematically by

and

$$
\mathrm{A}+\mathrm{A} \stackrel{k}{\rightarrow} \mathrm{A}
$$

$$
\mathrm{A}+\mathrm{A} \stackrel{k}{\rightarrow} \text { inert }
$$

respectively.

In the mean-field limit, i.e. the reaction-limited case, the kinetics of the process are described by a simple rate equation;

$$
\dot{c}_{\mathrm{A}}(t)=-k c_{\mathrm{A}}^{2}(t) \text {. }
$$

Here, $c_{\mathrm{A}}(t)$ is the concentration of A-particles at time $t$, and the dot denotes differentiation with respect to time. In this approximation, the two processes in $(7.9 a, b)$ are described by the same rate equation (7.10), and the different numbers of particles that result from a single reaction in each case can be easily taken into account in the reaction constant $k$. The solution of (7.10) is 


$$
c_{\mathrm{A}}(t)=\frac{1}{k t+1 / c_{\mathrm{A}}(0)},
$$

which is the mean-field prediction for the decay of concentration in the one-species annihilation process.

The diffusion-controlled process of one-species annihilation has been studied extensively [128, 330-335]. Peliti [336] has shown rigorously, using a field-theory approach, that the two processes $(7.9 a, b)$ are in the same universality class. The kinetics of the process has been derived by several authors [128, 332, 334, 336], and the theory includes rigorously proved results. The concentration decays as

$$
c_{\mathrm{A}}(t) \sim 1 / t^{d / 2}, \quad d<d_{\mathrm{c}}=2 .
$$

For $d<2$ the kinetics is anomalous, and $d_{\mathrm{c}}=2$ is the upper critical dimension above which the process is normal and decays according to the mean-field prediction of (7.11).

A simple scaling argument $[333,335]$ that stresses the role of diffusion and leads to the result of (7.12) is as follows. Imagine a domain in the system of linear size $L$. Initially there are in this domain of the order $c_{\mathrm{A}}(0) L^{d}$ particles, but, as the process goes on, the particles in the domain interact and annihilate in pairs. After a time $t \sim L^{2}$ (a characteristic time for a diffusing particle to get across the domain) all the particles in the domain have had the chance to interact and annihilate, leaving of the order of one particle in it. Thus at time $t$

$$
c_{\mathrm{A}}(t) \approx \frac{1}{L^{d}} \sim \frac{1}{t^{d / 2}}
$$

which is exactly the result given in (7.12). For $d>2$ the random walk performed by the particles is not recurrent, and therefore waiting a time $t \sim L^{2}$ is not sufficient for the completion of the process within the domain, and the above argument breaks. The mean-field result that $c_{\mathrm{A}}(t) \sim 1 / t$ is then valid. The argument is easily generalized to disordered media. The characteristic scaling of time is then $t \sim L^{d_{\mathrm{w}}}$ and upon the completion of the process in the domain, the residual concentration is $[330,331]$

$$
c_{\mathrm{A}}(t)=\frac{1}{L^{d_{\mathrm{f}}}} \sim \frac{1}{t^{d_{\mathrm{f}} / d_{\mathrm{w}}}}=\frac{1}{t^{d_{\mathrm{s}} / 2}} .
$$

Thus one gets the same result as in (7.13), only $d$ is replaced by $d_{\mathrm{s}}$. In addition to the numerical simulations [330], there have been some remarkable experiments of Kopelman et al. [41] on porous membranes, films, polymeric glasses and isotropic mixed crystals, confirming this result.

We mention that the process in $(7.9 a)$ describes, in a gross manner, the particlecoalescence model (PCM). A more detailed description of this aggregation is

$$
\mathrm{A}_{i}+\mathrm{A}_{j} \stackrel{k_{i, j}}{\longrightarrow} \mathrm{A}_{i+j} .
$$

Here $\mathrm{A}_{i}$ denotes a particle of mass $i$, and (7.14) symbolizes the event in which a particle of mass $i$ sticks to a particle of mass $j$, resulting in a particle of mass $i+j$. If we give up the identification of different particles according to their mass, and take all the reaction rates $k_{i, j}$ as equal to $k$, the aggregation process in (7.14) reduced to the representation in (7.9a). Smoluchowski [337] presented a solution of the kinetics of this aggregation in the mean-field limit, with a constant matrix of reaction rates, $k_{i, j}=k$. Indeed the total number of particles $\Sigma_{i} \mathrm{~A}_{i}$ decays as $1 / t^{2}$, in agreement with 
(7.11). This is in contrast with the result of the diffusion-limited case of (7.12) that $\Sigma_{i} \mathrm{~A}_{i} \sim 1 / t^{d / 2}$. The effect of diffusion is also apparent in the decay of monomers $\mathrm{A}_{1}$. While in the mean-field limit the number of monomers decays as $1 / t^{2}$, it can be shown that in the diffusion-limited case it decays as $1 / t^{3 / 2}$ (in $d=1$ ).

\subsection{Two-species annihilation and the effect of spatial fluctuations in concentration}

Another process of interest and wide applicability is that in which particles of two different species A and B combine and annihilate, or form a third inert species $[128,331]$. This two-species annihilation process is symbolically represented by

$$
\mathrm{A}+\mathrm{B} \stackrel{k}{\rightarrow} \text { inert. }
$$

In the mean-field approximation the kinetics is described by the rate equations

The conservation law

$$
\dot{c}_{\mathrm{A}}(t)=\dot{c}_{\mathrm{B}}(t)=-k c_{\mathrm{A}}(t) c_{\mathrm{B}}(t) .
$$

$$
c_{\mathrm{A}}(t)-c_{\mathrm{B}}(t)=\text { constant } \equiv \Delta
$$

derived from these equations reflects the fact that with each reaction that takes place the difference in the number of A-particles and B-particles remains unchanged. Equation (7.16) is readily solved with the aid of (7.17):

$$
c_{\mathrm{A}}(t)=\Delta\left[1-\frac{c_{\mathrm{A}}(0)}{c_{\mathrm{B}}(0)} \exp (-\Delta k T)\right]^{-1} .
$$

In the special case of equal initial concentrations, $c_{\mathrm{A}}(0)=c_{\mathrm{B}}(0)$, the exponential decay (of the minority species) is replaced by an algebraic decay

$$
c_{\mathrm{A}}(t)=\left(k T+\frac{1}{c_{\mathrm{A}}(0)}\right)^{-1} \text {. }
$$

This special case of symmetry has attracted much interest.

In considering the non-classical behaviour of the diffusion-controlled two-species annihilation process, spatial fluctuations in the concentration of the reactants must be taken into account [333]. Consider the case of equal initial concentrations, $c_{\mathrm{A}}(0)=c_{\mathrm{B}}(0)=c(0)$. In a domain of linear size $L$ there are initially of the order of $c(0) L^{d} \pm\left[c(0) L^{d}\right]^{1 / 2}$ A-particles (and likewise for B-particles). The fluctuations in the number of particles are due to the totally random distribution of the reactants assumed in the initial state. After a time $t \sim L^{2}$ the particles in the domain have had the chance to interact and annihilate in pairs, leaving a residue of particles of that species that was initially in the majority (owing to fluctuations in concentration). Thus the number of particles left is of order $\left[c(0) L^{d}\right]^{1 / 2}$. Note that the conservation law $c_{\mathrm{A}}(t)-c_{\mathrm{B}}(t)=$ const is implicitly used in this argumentation. One is allowed to use this conservation law because it is generally valid, by virtue of the pairwise annihilation, and not restricted to the mean-field case. The concentration then decays as

$$
c(t) \sim \frac{\left[c(0) L^{d}\right]^{1 / 2}}{L^{d}} \sim c(0) t^{-d / 4}, \quad d \leqslant d_{\mathrm{c}}=4 .
$$

The formation of domains, owing to the spatial fluctuations in concentration, is essential to the result in (7.20). The reaction is largely inhibited because of these domains, as it takes place only along the boundaries between domains. Figure 42 


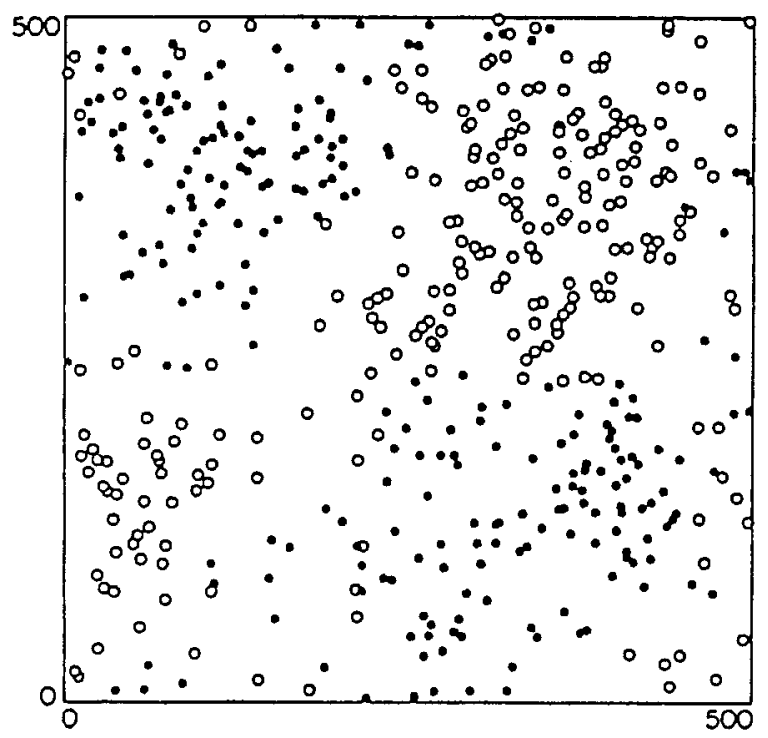

Figure 42. Particle and antiparticle (A and B) positions at $t=1000$ on a $d=2,500 \times 500$ square system. The initial condition was a uniform distribution with concentration $c=0.05$. (After Toussaint and Wilczek [128].)

shows the formation of domains as seen in computer simulations of the two-species annihilation process in $d=2$.

The decay law given in (7.20) is valid for $d \leqslant 4$, and $d_{\mathrm{c}}=4$ is the upper critical dimension above which the mean-field result holds. One way of understanding why the approach leading to (7.20) fails for $d>4$ is the following. Since for $d>2$ the random-walk trail performed by a particle is no longer recurrent, the probability that a particle interacts with a single particle enclosed in a volume $L^{d}$ scales like $L^{d-2}$. Assuming that domains develop, because of the spatial fluctuations in concentration, we should expect to have of the order of $L^{d / 2}$ particles in a domain of linear size $L$ belonging to the species that is (locally) in the majority. Then, a particle of the opposite species has a probability $L^{2-d} \times L^{d / 2}$ of interacting and annihilating upon crossing the domain. For $d>4$ this probability scales to zero. Thus for $d>4 \mathrm{a}$ domain of particles of one species will be 'transparent' to the opposite species, and the formation of domains is impossible, invalidating the approach that led to (7.20).

The striking differences between the anomalous kinetics of the one- and twospecies annihilation processes have led K. Kang to propose the $n$-species annihilation model, which is a reaction model that interpolates smoothly between these two important cases. The system consists of $n$ different species $\mathrm{A}_{1}, \mathrm{~A}_{2}, \ldots, \mathrm{A}_{n}$ that interact in pairs, annihilating or combining to form some other, inert, species:

$$
\mathrm{A}_{i}+\mathrm{A}_{j} \stackrel{k}{\rightarrow} \text { inert }, \quad i \neq j .
$$

For $n=2$ the problem reduces to that of the two-species annihilation process. For $n \rightarrow \infty$ the constraint that a particle does not interact with its own species becomes negligible, and the process converges to the one-species annihilation case. Thus on increasing $n$ from 2 to $\infty$ one gets a series of systems that interpolates between twoand one-species annihilation processes. 
The kinetics of this problem in the mean-field limit as well as in the diffusioncontrolled cases was studied by Ben-Avraham and Redner [338]. For the mean-field limit with equal initial concentrations the decay goes like $1 / t$. In the diffusioncontrolled case they found that fluctuations arising from the discrete nature of the reactants must be taken into account. This is in addition to the spatial fluctuations in concentration and the scaling due to diffusion, which also have an effect. Their result is that the concentration decays as

$$
c(t) \sim c(0)^{1 /[2(n-1)]} t^{-(d / 2)[1-1 / 2(n-1)]},
$$

with an upper critical dimension $d_{\mathrm{c}}=2+2 /(2 n-3)$. This result indeed interpolates between the expressions given in (7.12) and (7.20) for the one- and two-species annihilation.

\section{Further cases of diffusion}

\subsection{Diffusion of particles with hard-core interactions}

It has been realized from experiments by Laibowitz and Gefen [311], that the transport properties of real materials with a fractal structure cannot be explained simply in terms of random-walk models where the walkers do not interact with each other. Most interaction schemes are difficult to investigate [339], and research has focused [340-347] on the case of diffusing particles with a repulsive hard-core interaction.

Diffusion of particles with hard-core interactions in Euclidean space has been studied by several authors [340-347]. While for $d>1$ diffusion is still normal even in the hard-core interaction case, diffusion is anomalous in $d=1$. The diffusion of a labelled or tagged particle (tracer diffusion) is greatly inhibited by its repulsing neighbours. The mean displacement of a tagged particle follows the asymptotic law

$$
\left\langle r^{2}\right\rangle=\frac{2}{\pi^{1 / 2}} \frac{1-c}{c} t^{1 / 2} \quad \text { as } t \rightarrow \infty,
$$

meaning that diffusion of a tracer particle is anomalous with $d_{\mathrm{w}}=4$. For small times $t$ the tagged particle does not 'see' its neighbours, and its diffusion is normal:

$$
\left\langle r^{2}\right\rangle=2(1-c) t \text { for small } t .
$$

The anomalous diffusion of a tagged particle in $d=1$ has consequences for the problem of trapping of tagged particles on the line in the presence of another type of particle which cannot be trapped. Bunde et al. [348] studied the limit of a single trap as well as the case of a finite concentration [349] of traps. For the single-trap limit they found that the trapping rate scales as $t^{-1 / 2}$ for the short times and as $t^{-3 / 4}$ in the asymptotic large-time limit. This is in accordance with (8.1) and (8.2), since the trapping rate is proportional to the time derivative of $1 /\left\langle r^{2}(t)\right\rangle^{1 / 2}$. For the case of a finite concentration of traps the stretched exponential decay law of the survival probability discussed in section 7.1 changes to

$$
S(t) \sim \exp \left[-p^{4 / 5}(1-c)^{2 / 5} t^{1 / 5}\right] .
$$

Here $p$ is the concentration of traps and $c$ is the concentration of the particles. The slower decay of $S(t)$, as compared with (7.1), is due to the slower anomalous diffusion motion of a tagged particle, (8.1). 
The study of diffusion of particles with hard-core interactions has been extended from Euclidean spaces to DLA (in $d=2$ ) [350] and to percolation clusters in $d=2$ and $d=3$ (see reference [351], and C. Amitrano, A. Bunde and H. E. Stanley, 1986, unpublished). Amitrano et al. [350] found that in DLA the long-time behaviour can be described by

$$
\left\langle r^{2}\right\rangle \sim\left[(1-c) f_{\mathrm{T}}(c) t\right]^{2 / d_{\mathrm{w}}} .
$$

Here $f_{\mathrm{T}}(c)$ is a function that describes correlations of consecutive jumps of the tracer particles and generalizes the Euclidean results, see, for example, [340] for two- and three-dimensional lattices. For $c$ close to $1, f_{\mathrm{T}}(c) \approx 1-c$. The anomalous-diffusion exponent $d_{\mathrm{w}}$ in (8.4) is the same as for the non-interacting case, i.e. hard-core interactions do not change the fractal dimensionality of the walks in DLA. This holds true for all concentrations $c$. However, for concentrations close to $c=1$

$$
\left\langle r^{2}\right\rangle=g\left(\frac{t}{t_{x}}\right), \quad t_{x}=\frac{c^{2}}{(1-c)^{2}},
$$

where

$$
g(x) \sim \begin{cases}x^{1 / 2} & (x \ll 1), \\ x^{2 / d_{w}} & (x \gg 1) .\end{cases}
$$

Amitrano et al. [340] explained this result by the fact that for short times the tagged particle sees a finite quasi-one-dimensional section of the DLA, therefore reproducing the one-dimensional law (8.1). In the asymptotic large-time limit the particle senses the fractal structure of the substrate and the limit (8.4) is recovered. Similar results have been reported for percolation clusters (see reference [351] and C. Amitrano, A. Bunde and H. E. Stanley, 1986, unpublished). The effect of a uniform bias field on diffusing particles with hard-core interactions has been studied in $d=1$ dimensions by R. Kutner and H. van Beijeren (preprint, 1987) and for random networks by R. Ramaswamy and M. Barma (preprint, 1987).

(a)

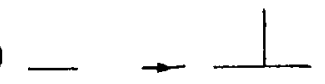

(b)

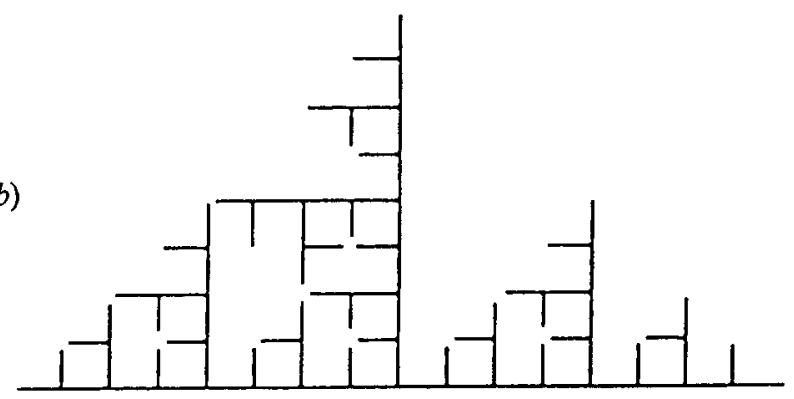

Figure 43. Deterministic tree: $(a)$ generation law; $(b)$ the tree shown to four generations. 
(a) $\rightarrow-1$

(b)

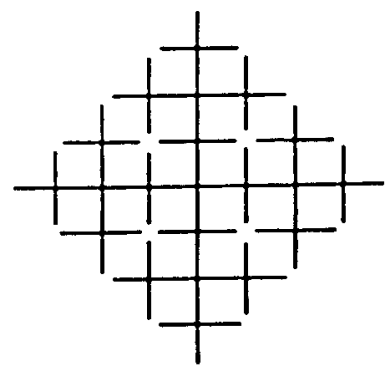

Figure 44. Deterministic tree: $(a)$ generation law; $(b)$ the tree shown to four generations.

\subsection{Diffusion on deterministic fractals}

The subject of transport of loopless, finitely ramified, statistical fractals has been reviewed in section 4 . The main results are that the anomalous-diffusion exponent is $d_{\mathrm{w}}^{l}=d_{l}+1,(4.5)$, and that the probability distribution of being at a chemical shell $l$ at time $t$ is $P(l, t)=A t^{-d_{s} / 2} \exp \left[-a\left(l / t^{1 / d_{\mathrm{w}}^{l}}\right)^{v}\right]$, with $v=d_{\mathrm{w}}^{l}\left(d_{\mathrm{w}}^{l}-1\right)$, (4.20). We now present two examples of loopless, finitely ramified, deterministic fractals, and show that the above results are valid.

These two examples are shown in figures 43 and 44. It is evident from the figures that for both fractals $d_{\mathrm{f}}=d_{l}$, and $\tilde{\nu}=1$. This feature is not general to deterministic loopless fractals. From the definition of $d_{\mathrm{f}}$ it follows that the fractal dimension of the tree in figure 43 is $d_{\mathrm{f}}=\ln 3 / \ln 2$, and that of the tree in figure 44 is $d_{\mathrm{f}}=\ln 4 / \ln 2=2$.

Owing to the exact self-similarity of these fractals, one can rigorously prove [274] that $d_{\mathrm{w}}^{l}=d_{l}+1$. This is also confirmed by an exact enumeration of the walks on the trees. Results are shown in figure 45 . The exact enumeration provides us with results for $P(l, t)$. The structure of $P(l, t)$ for the tree of figure 43 is shown in figure 46 . The envelope of $P(l, t)$ scales exactly as proposed in (4.20). Results for both fractal trees are presented in figure 47.

Transport properties on deterministic infinitely ramified fractals are harder to analyse, and there are no exact results for this problem. Ben-Avraham and Havlin [53] have derived rigorous bounds for the transport exponents of a family of exact fractal lattices with an infinite ramification. The fractal lattices belonging to this family are characterized by two parameters, $b$ and $x$. The generator or genus of each lattice is obtained from a $d$-dimensional hypercube subdivided to $b^{d}$ smaller hypercubes. The parameter $x(0 \leqslant x \leqslant 1)$ is a measure of the number of hypercubes that actually belong to the generator (the $b^{d}$ hypercubes are diluted). For example, in $d=2$ the genus consists of $b^{2}$ squares diluted so that there are $b(1-x)$ rows of 


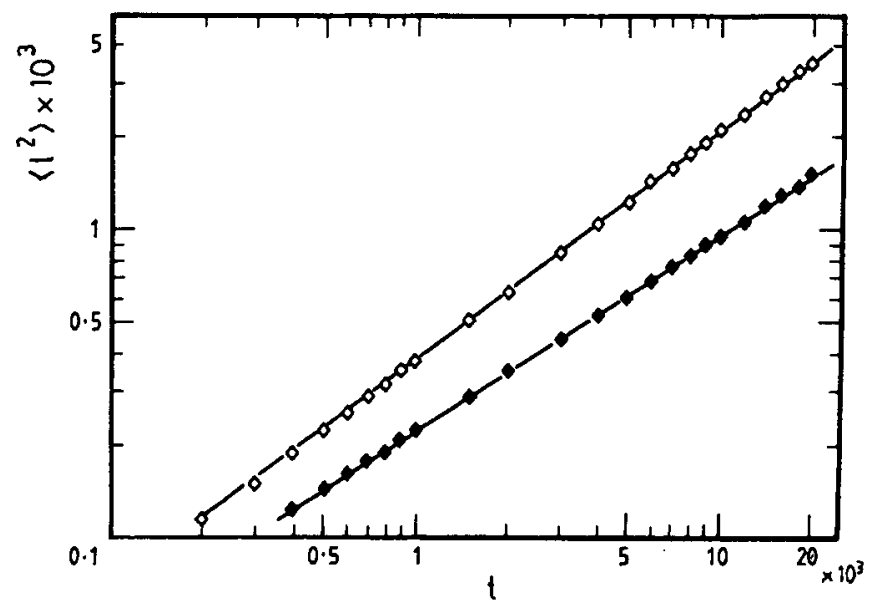

Figure 45. Plot of $\left\langle l^{2}\right\rangle$ as a function of $t$ for diffusion on the fractals of figures $43(\diamond)$, and $44(\diamond)$. The dots represent the data from exact enumeration, and the solid curves were traced according to $d_{\mathrm{w}}^{l}=d_{l}+1$.

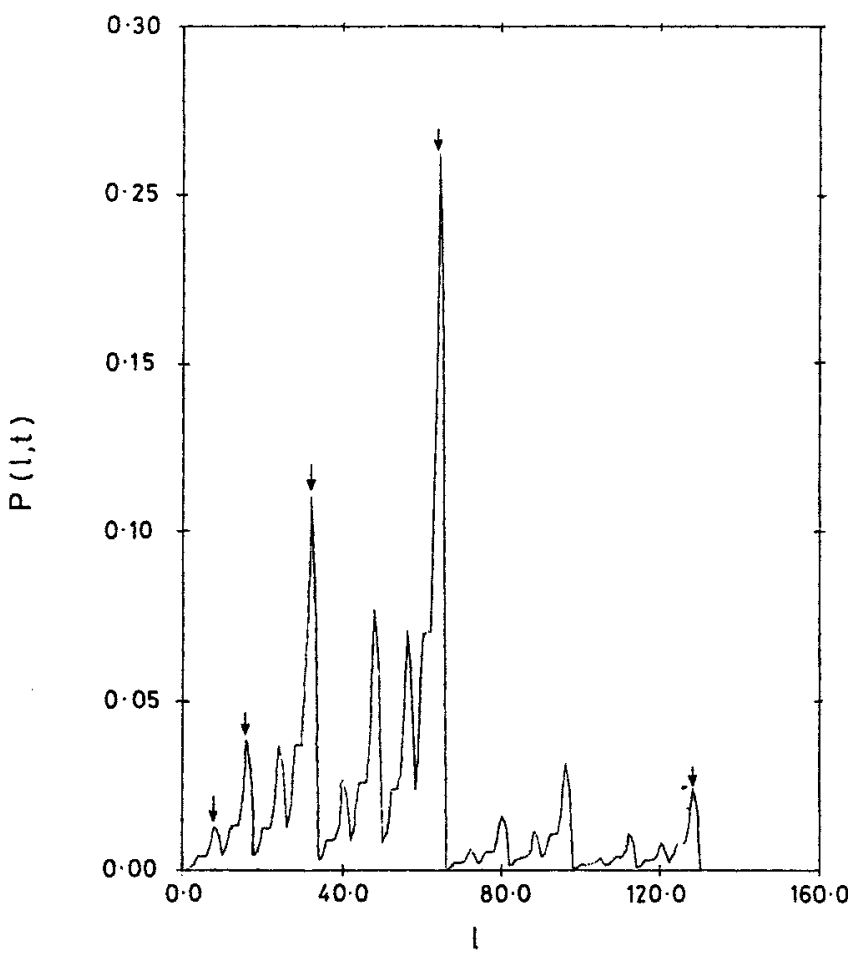

Figure 46. The probability distribution, $P(l, t)$, for diffusion on the fractal of figure 43 . The envelope points are marked by the arrows. 


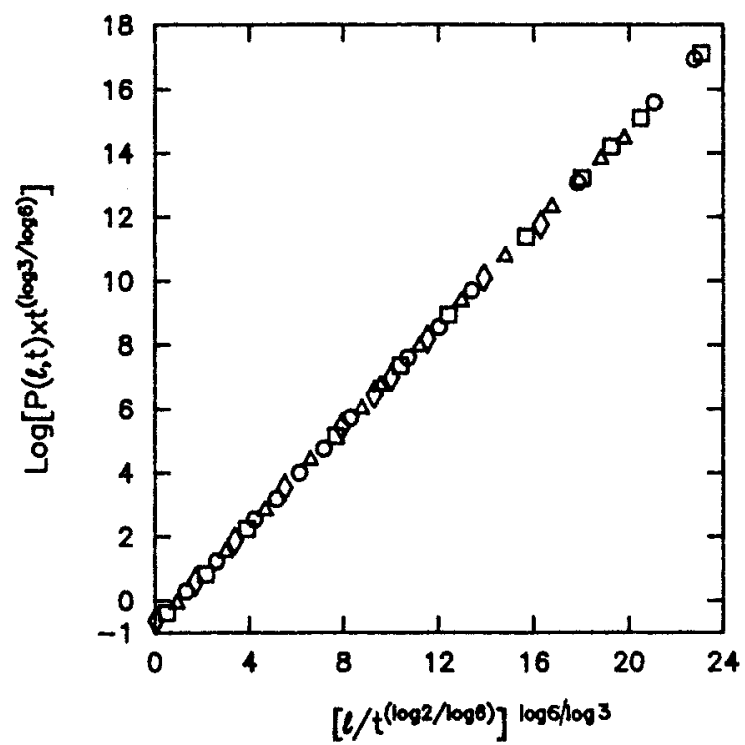

(a)

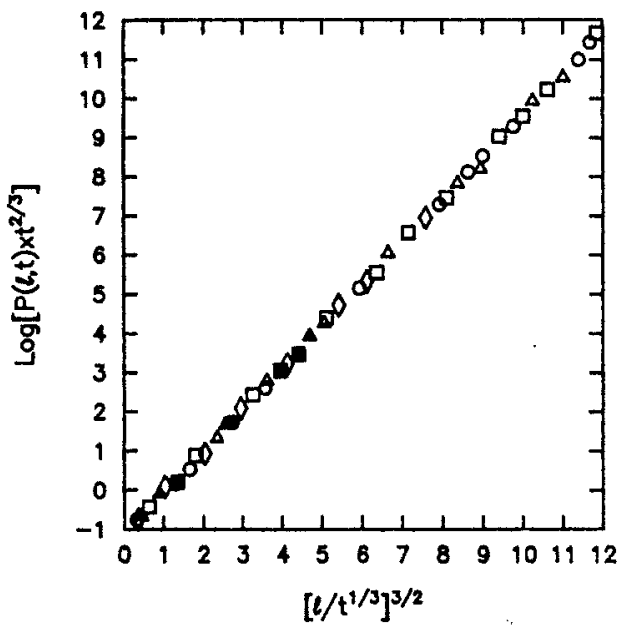

(b)

Figure 47. Scaling of $P(l, t)$ for the deterministic fractals of figures $(a) 43(b)$ and $(b) 44(b)$. The linear shape of the resulting curves confirms (4.20).

$b$ connected squares, and the remaining $b x$ rows contain $b(1-x)$ squares each (figure 48).

The fractal dimension of a two-dimensional lattice $\uparrow$ with the parameters $b$ and $x$ is

$$
d_{\mathrm{f}}=2+\frac{\ln \left(1-x^{2}\right)}{\ln b} .
$$

For the resistance exponent $\tilde{\zeta}$ the rigorous bounds 
(a)

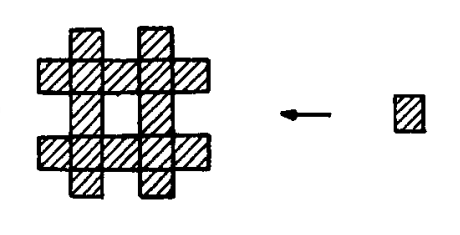

(b)

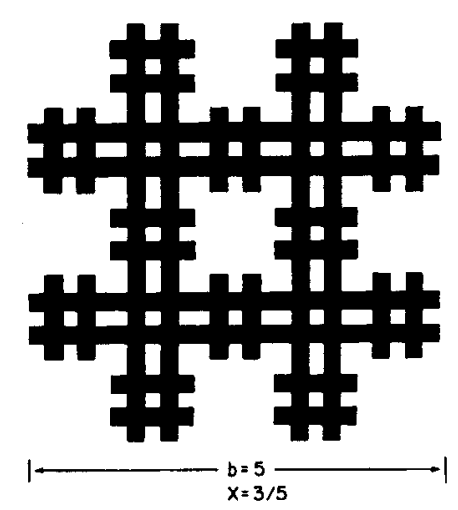

Figure 48. (a) Genus of a two-dimensional fractal lattice with $b=5$ and $x=\frac{3}{5}$. (b) The lattice is shown to two generations.

$$
\ln \left(\frac{1-x+x^{2}}{1-x}\right) / \ln b \leqslant \tilde{\zeta} \leqslant \ln \left(\frac{1}{1-x}\right) / \ln b
$$

are derived. From the Einstein relation $d_{\mathrm{w}}=d_{\mathrm{f}}+\tilde{\zeta}$ bounds of $d_{\mathrm{w}}$ follow:

$$
2+\frac{\ln \left[(1+x)\left(1-x+x^{2}\right)\right]}{\ln b} \leqslant d_{\mathrm{w}} \leqslant 2+\frac{\ln (1+x)}{\ln b} .
$$

The above bounds become narrower and coincide when $x \rightarrow 0$ or $b \rightarrow \infty$. Numerical simulations for diffusion on fractal lattices belonging to the family of figure 48 have been performed by Movshovitz [352] and by J. Given (private communication). Their results are consistent with the bounds of (8.9).

\subsection{Self-avoiding walks on fractals}

A problem that is closely related to the problem of diffusion (random walks) in disordered media is that of self-avoiding walks (SAW) [353-360, 400] on fractals. The interest in this problem stems from its analogy with the $n=0$ vector model $[10,361]$. The configurational properties of SAWs in disordered media are largely influenced by the geometrical properties of the backbone of the substrate. Because of the excluded-volume interaction on SAWs, a walk cannot exit from a dangling end once it has previously entered it. Thus the SAW lies mainly on the backbone. This is in contrast with random walks, which explore all parts of the substrate.

The end-to-end exponent $\nu$ for SAWs is defined [362] by

$$
\left\langle R_{N}^{2}\right\rangle \sim N^{2 \nu}
$$

where $\left\langle R_{N}^{2}\right\rangle$ is the mean-square end-to-end distance of a SAW consisting of $N$ steps. The value of $\nu$ for Euclidean lattices is very successfully predicted by the mean-field Flory theory $[10,363]$ 
(a)

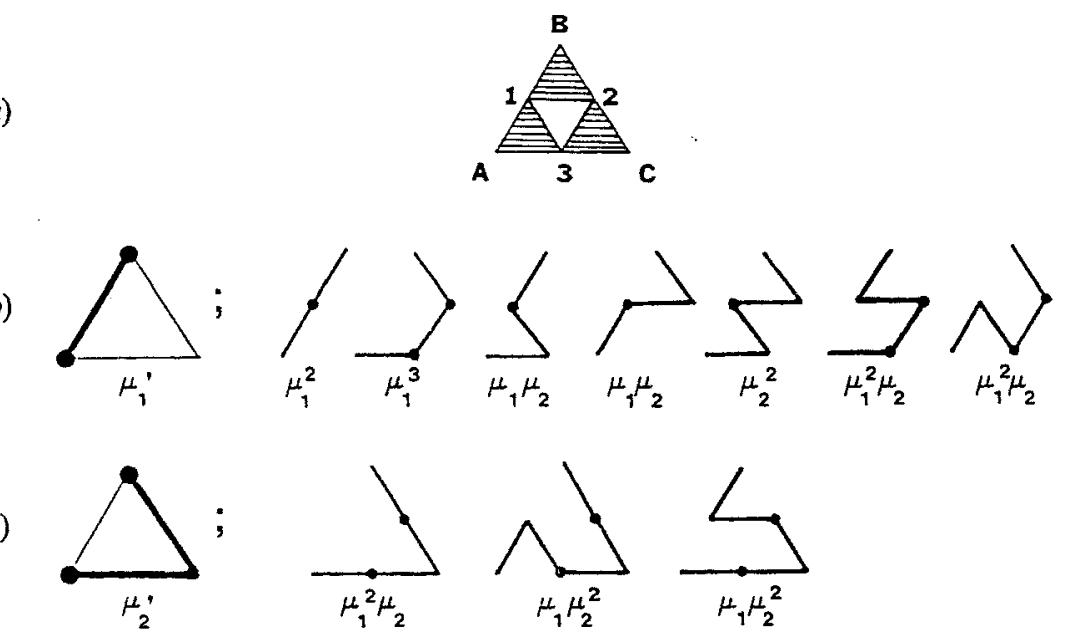

Figure 49. (a) Sierpinski gasket. (b) and (c) Coarse graining of SAWs on the gasket.

$$
\nu_{\mathrm{F}}=3 /(d+2), \quad d \leqslant d_{\mathrm{c}}=4,
$$

where the subscript $\mathrm{F}$ stands for Flory. Equation (8.11) agrees with the known exact results in $d=1,2$ [364] and $d=d_{\mathrm{c}}=4$. The question as to whether the Flory prediction is exact for $d=3$ is still open. The validity of the Flory approach can be tested for SAWs on finitely ramified exact fractal lattices. The end-to-end exponent $\nu$ can be obtained exactly using a renormalization-group procedure, and compared with the prediction from a Flory theory.

The exact real-space renormalization-group (RSRG) technique for SAWs on the Sierpinski gasket $[354,358-360]$ consists in a decimation procedure on the points 1,2 and 3 of figure $49(a)$. Let $\mu_{1}^{\prime}$ be the fugacity associated with a SAW lying between the vertices A and B and that does not touch C (figure $49(b)$ ) and let $\mu_{2}^{\prime}$ be the fugacity associated with a SAW lying from A to B and passing through C (figure $49(c)$ ). These rescaled fugacities $\mu_{1}^{\prime}$ and $\mu_{2}^{\prime}$ can be obtained from the decimation of the inner vertices of the gasket (vertices 1, 2 and 3 in figure $49(a)$ ) and represented in terms of $\mu_{1}$ and $\mu_{2}$, the analogous fugacities for a SAW in the gasket before rescaling.

Figures $49(b, c)$ show the different configurations contributing to $\mu_{1}^{\prime}$ and $\mu_{2}^{\prime}$ :

$$
\left.\begin{array}{l}
\mu_{1}^{\prime}=\mu_{1}^{2}+\mu_{1}^{3}+2 \mu_{1} \mu_{2}+\mu_{1}^{2}+2 \mu_{1}^{2} \mu_{2}, \\
\mu_{2}^{\prime}=\mu_{1}^{2} \mu_{2}+2 \mu_{1}, \mu_{2}^{2} .
\end{array}\right\}
$$

The only non-trivial point is

$$
\mu_{2}^{*}=0, \quad \mu_{1}^{*}=\frac{\sqrt{ } 5-1}{\sqrt{ } 2} .
$$

The eigenvalues around the fixed point are

$$
\lambda_{1}=\mu_{1}^{* 2}<1, \quad \lambda_{2}=2 \mu_{1}^{* 2}+3 \mu_{1}^{* 2}>2 .
$$

A study of the flow in the $\left(\mu_{1}, \mu_{2}\right)$ plane performed by Rammal et al. [354] yielded

$$
\nu=\frac{\ln 2}{\ln \left(2 \mu_{1}^{* 2}+3 \mu_{1}^{* 2}\right)} \approx 0.798 .
$$


Table 8. Exponents characterizing SAWs on percolation clusters at criticality used in the text.

\begin{tabular}{|c|c|c|c|c|c|}
\hline$d$ & $\tilde{\nu}$ & $\begin{array}{c}\nu_{l} \\
(8.31)\end{array}$ & $\nu=\nu_{l} \tilde{\nu}$ & $\begin{array}{c}\nu_{\mathrm{F}} \\
(8.26)\end{array}$ & $\begin{array}{c}\nu \\
\text { (regular } \\
\text { lattices }\end{array}$ \\
\hline 2 & 0.88 & 0.864 & $\begin{array}{l}0.77 \\
0.767^{\mathrm{a}}\end{array}$ & 0.69 & 0.75 \\
\hline 3 & 0.75 & 0.89 & $\begin{array}{l}0.67 \\
0.65 \text { [353] }\end{array}$ & 0.57 & 0.6 \\
\hline 4 & 0.64 & 0.936 & 0.59 & 0.49 & 0.5 \\
\hline 6 & $\frac{1}{2}$ & 1 & $\frac{1}{2}$ & $\frac{1}{2}$ & 0.5 \\
\hline
\end{tabular}

${ }^{\mathrm{a}}$ For large-cell RSRG results [401].

This result has also been dervied by Klein and Seitz [359]. A similar approach can be applied to the three-dimensional Sierpinski gasket, yielding $\nu=0.729$ [354].

Rammal, Toulouse and Vannimenus [354] presented a Flory theory for SAWs on fractals that yielded

$$
\nu_{\mathrm{F}}=\frac{1}{d_{\mathrm{f}}^{\mathrm{BB}}} \frac{3 d_{\mathrm{s}}^{\mathrm{BB}}}{d_{\mathrm{s}}^{\mathrm{BB}}+2} .
$$

The exponents $d_{\mathrm{f}}^{\mathrm{BB}}$ and $d_{\mathrm{s}}^{\mathrm{BB}}$ are respectively the fractal and fracton dimensions of the backbone. The presence of the backbone exponents in (8.16) reflects the fact that a SAW on a percolation cluster explores only the backbone of the cluster.

Since the Sierpinski gasket has no dangling ends, $d_{\mathrm{f}}^{\mathrm{BB}}=d_{\mathrm{f}}=\ln (d+1) / \ln 2$, and $d_{\mathrm{s}}^{\mathrm{BB}}=2 \ln (d+1) / \ln (d+3)$. Substituting in (8.16) the exact numerical values for $d_{\mathrm{f}}^{\mathrm{BB}}$ and $d_{\mathrm{s}}^{\mathrm{BB}}$ yields $\nu_{\mathrm{F}}=0.768$ for $d=2$ and $\nu_{\mathrm{F}}=0.654$ for $d=3$. These results disagree with the exact numerical values of $\nu(d=2)=0.798$ and $\nu(d=3)=0.729$ obtained from renormalization-group theory. This supports the argument that a Flory theory is not exact for SAWs in every dimension.

The question of SAWs on percolation clusters is of interest, and is rather controversial [353, 355-359]. Kremer [353] reports that numerical simulations of SAWs on the infinite percolation cluster for $p>p_{\mathrm{c}}$ indicate that the end-to-end exponent $\nu$ is the same as for Euclidean lattices. For $p=p_{\mathrm{c}}$ the value of $\nu$ changes. Harris [355] argues that for averages over all clusters the value of $\nu$ is the same as for Euclidean space, even in the case $p=p_{\mathrm{c}}$. Derrida [357], on the other hand, claims that $\nu$ is modified even by the presence of weak disorder, and that its value is different from normal space for all $p$.

The Flory theory, (8.16), has the following interpretation for SAWs on percolation clusters. For $p=p_{\mathrm{c}}$ the critical exponents $d_{\mathrm{s}}^{\mathrm{BB}}$ and $d_{\mathrm{f}}^{\mathrm{BB}}$, describe the incipient infinite percolation cluster. For $p>p_{\mathrm{c}}, d_{\mathrm{s}}^{\mathrm{BB}}$ and $d_{\mathrm{f}}^{\mathrm{BB}}$ characterize the incipient infinite percolation clusters for length scales $L$ smaller than the correlation length $\xi$. When $L \gg \xi$ the cluster is homogeneous, and is characterized by the normal Euclidean exponents $d_{\mathrm{s}}^{\mathrm{BB}}=d_{\mathrm{f}}^{\mathrm{BB}}=d$, and (8.26) reduces to (8.11). Thus the Flory theory agrees with Kremer [353] that SAWs have the same end-to-end exponent in percolation when $p>p_{\mathrm{c}}$ (for $L \gg \xi$ ) as for Euclidean lattices. The numerical values of $\nu_{\mathrm{F}}$ arising from (8.16) for SAWs on percolation clusters are presented in table 8 . 
In deriving (8.16), an assumption has been made that the distribution probability of random walks on a disordered medium scales like $P(r, t) \sim \exp \left(-r^{d_{\mathrm{w}}} / t\right)$. However, from the discussion in section 3.5, it is plausible that $P(r, t) \sim \exp \left[\left(r / t^{1 / d_{\mathrm{w}}}\right)^{u}\right]$, where $u$ is not necessarily equal to $d_{\mathrm{w}}$. Moreover, the Flory arguments can be carried out either in normal Pythagorean space or in chemical space. In the latter case one uses $P(l, t) \sim \exp \left[-\left(l / t^{1 / d_{\mathrm{w}}^{l}}\right)^{v}\right]$ for the density distribution. In the following the chemical space is used, since the Flory results in this case are in agreement with the limiting exact results of percolation in $d=1$ and in $d=6$. The Flory approximation, using normal Pythagorean space, fails in these cases. The Flory expression for the free energy of a SAW is (dropping all unimportant prefactors)

$$
F \approx \frac{N^{2}}{l^{d_{l}}}+\left(\frac{l}{N^{1 / d_{\mathrm{w}}^{l}}}\right)^{v} .
$$

Here $d_{l}$ and $d_{\mathrm{w}}^{l}$ are critical exponents describing the backbone and the diffusion on it in the chemical space respectively. Define and end-to-end exponent $\nu_{1}$ for a SAW in chemical space by

$$
l \sim N^{\nu_{l}}
$$

Since $R \sim l^{\tilde{\nu}}$ (section 3.4), one obtains $R \sim N^{\tilde{\nu} \nu_{l}}=N^{\nu}$, and it follows that

$$
\nu=\tilde{\nu} \nu_{l}
$$

Note that, since the SAW is a subset of the backbone, $\nu \geqslant 1 / d_{\mathrm{f}}$. On the other hand, $\nu_{l} \leqslant 1$; thus

$$
\frac{1}{d_{\mathrm{f}}}=\frac{\tilde{\nu}}{d_{l}} \leqslant \nu=\nu_{l} \tilde{\nu} \leqslant \tilde{\nu} .
$$

Minimizing the free energy $F(8.27)$ yields the Flory-type result

$$
\nu_{l}=\frac{2+v / d_{\mathrm{w}}^{l}}{v+d_{l}} .
$$

Table 8 shows the numerical values of $\nu_{l}$ and $\nu$ obtained from (8.19) and (8.21), using the estimated values of $v, d_{\mathrm{w}}^{l}$ and $d_{l}$ for percolation clusters (section 3.6). Note that the numerical values of $\nu$ from (8.19) are larger than the corresponding end-toend exponents of SAWs on regular lattices. In contrast, $\nu_{\mathrm{F}}$ from (8.16) gives smaller values than in regular space.

As for the question of the validity of the Flory approach for the evaluation of $\nu$, the results are inconclusive. For the Sierpinski gasket, $\nu$ for SAWs in $d=2$ and $d=3$ is known exactly, and so are the exponents $d_{l}$ and $d_{\mathrm{w}}^{l}$. However, the value of $v$ is unknown in both cases (apart from simulations). Assuming that the Flory result of (8.21) holds, we get $v=1.995$ for the Sierpinski gasket in $d=2$, and $v=1.584$ for the gasket in $d=3$. These values are in general agreement with numerical simulations $[113,114]$ and with $v=d_{\mathrm{w}} /\left(d_{\mathrm{w}}-1\right)$ of section 2.4. An exact solution for the exponent $v$ will provide us with the answer to the question as to whether the Flory result is exact or not on the Sierpinski gasket, thus shedding light on the more fundamental question of the validity of the Flory approach for regular $d=3$ space. 


\subsection{Diffusion in other types of disordered systems}

There are various cases of diffusion in other disordered systems that have not been reviewed here. It is hardly possible to include all of the enormous amount of examples. A short list is included in the following.

The problem of transport in a random mixture of two types of conductors has been given much attention [365-372]. Indeed, percolation is a particular case of this general problem, where one of the types of 'conductors' is a perfect insulator. The system has been used as a model of ionic conductors mixed with a dispersed insulating phase [142], and resistor-superconducting mixtures such as thin films of superconducting material deposited on a normal substrate [373]. Diffusion on kinetic-gelation [374, 375] clusters has been studied experimentally by R. Bansil and L. Carvalho (1987, preprint) and theoretically by N. Bahadur, H. J. Herrmann and D. P. Landau (preprint, 1987). They have found that diffusion is anomalous and that the value of $d_{\mathrm{w}}$ cannot be distinguished from that of diffusion in threedimensional percolation clusters at criticality. Diffusion on percolation clusters in the presence of random fields was studied numerically by Pandey [377]. Anomalous diffusion in any dimension in the presence of long-range correlated random forces was found by J. P. Bouchaud, A. Comtet, A. Georges and P. Le Doussal (preprint, 1987).

The theory of anomalous diffusion in disordered systems has been employed in the study of the substrates generated by simple random walks (see references [85, 213, 376] and D. Movshovitz and S. Havlin, preprint, 1987), in the study of polymers using the self-avoiding-walk model [67,378-381], and in the derivation of some novel features of the overlapping Lorentz gas [382].

Anomalous diffusion has also been studied for dynamically disordered systems (A. Nitzan, S. D. Druger and M. A. Ratner, 1987, preprint) and in stirred percolation [383, 384]. The problem is relevant to the electrical conductivity of water-in-oil microemulsions [385]. Diffusion on directed percolation [386] has been considered by Vicsek et al. [387] and by Stephen [388]. Other substrates in which anomalous diffusion has been studied are hierarchical lattices [389], multifractals $(\mathrm{H}$. Weissmann and S. Havlin, 1987, preprint, P. Meakin, 1987, preprint) and diffusion near absorbing fractals (M. E. Cates and T. A. Witten, 1987, preprint). An exact solution to the problem of diffusion on a quasi-periodic chain has been provided by Khanta and Stinchcombe [390] using a decimation method involving scaling by an irrational length factor. This provides some understanding of the problem of transport in quasicrystals [391].

Charge transport in amorphous semiconductors also involves in many cases amorphous diffusion and/or percolation, and has been very extensively studied and reviewed $[108,286,287,392-396]$ in connection with the electrical conductivity and photoconductivity of these materials. The basic quantity from which these properties can be derived is the configurational average of the probability density $P(\mathbf{r}, t)$, introduced in section 2.4, that a particle will be found distance $\mathbf{r}$ from its starting point after time $t$. Two extreme types of problems have been treated in detail. One of these is where the charge carriers move through the system in extended states, i.e. as free particles, subject to trapping in, and release from, a set of potential wells of random depth (see section 5.3) [397]. It has been shown that this problem can be treated exactly by the use of a distinction of waiting times and the continuous-time random-walk method. The other problem is that known as $r$-percolation [392], in which the charge carriers move between states of the same energy localized at sites 
distributed randomly through the medium, with the transition rate between any two sites depending only on the distance $R$ between them, and usually decaying exponentially with $R$. A much more realistic but difficult problem is that in which the carriers move between localized states of different energy, with the transition rate depending on the energy difference as well as on the distance between the sites, so that it is no longer symmetrical for transitions in the two directions. For this sort of problem, there is no obvious way of defining critical exponents or fractal dimensions.

\section{Acknowledgements}

We wish to acknowledge useful collaborations with A. Aharony, S. Alexander, A. Bunde, R. C. Ball, M. Dishon, Z. Djorjevic, Y. Glaser, H. Harder, D. Hong, J. E. Kiefer, R. Leyvraz, I. Majid, B. B. Mandelbrot, P. Meakin, D. Movshovitz, R. Nossal, S. Redner, H. E. Roman, H. Sompolinsky, H. E. Stanley, D. Stauffer, H. Taitelbaum, B. L. Trus, I. Webman, G. H. Weiss and H. Weissman. We also appreciate critical reading of the manuscript by A. Aharony, A. Bunde, W. Dieterich, C. Domb, H. Gould, V. Halpern, B. Harris, J. Klafter, R. Kopelman, F. Leyvraz, S. Redner, H. E. Stanley, D. Stauffer, H. Taitelbaum, I. Webman, G. H. Weiss, N. Wiser and H. Weissman and we are grateful to D. A. Lavenda for typing the manuscript. This work was partly supported by a Minerva Fellowship.

\section{Appendix: Numerical methods}

The study of diffusion on random media has lead to the development of various sophisticated numerical methods: Monte Carlo procedures and exact enumerications. Frequently, the numerical work is double, requiring simulations of the substrate, as well as of the diffusion process. Also, the diffusion process can be realized by different kinds of random walkers or 'ants' [398]. These different kinds of walkers arose partly because of numerical convenience and partly in order to model specific physical situations as, for example, 'termites' in the study of superconducting clusters [367, 370].

\section{A.1. Monte Carlo methods}

A straightforward numerical approach to the problem of diffusion on random media requires a double Monte Carlo procedure: one for simulating the substrate, the other for the simulation of diffusion itself. The methods for the simulation of the substrate depend, of course, on the substrate itself. For percolating clusters the well known cluster-growth method [175, 184] and the Hoshen-Kopelman algorithm [399] are most useful. To these, one can add the very recent methods of cluster burning [179] for the generation of the backbones of percolation clusters. Monte Carlo procedures for the simulation of DLA clusters [63, 228] are also continuously improving. With the modern sophisticated algorithms exploiting the equivalence of DLAs to electrostatics [119], giant clusters of millions of sites are currently generated. These are just a few examples. Diffusion itself is simulated by a random walker. Different weights can be assigned for a step to the unblocked directions, giving rise to several types of walkers, 'ants' or 'termites', some of which are reviewed in section A.3.

Some numerical techniques avoid, or reduce to some extent, the number of Monte Carlo steps required because of the double simulation procedure. As an 


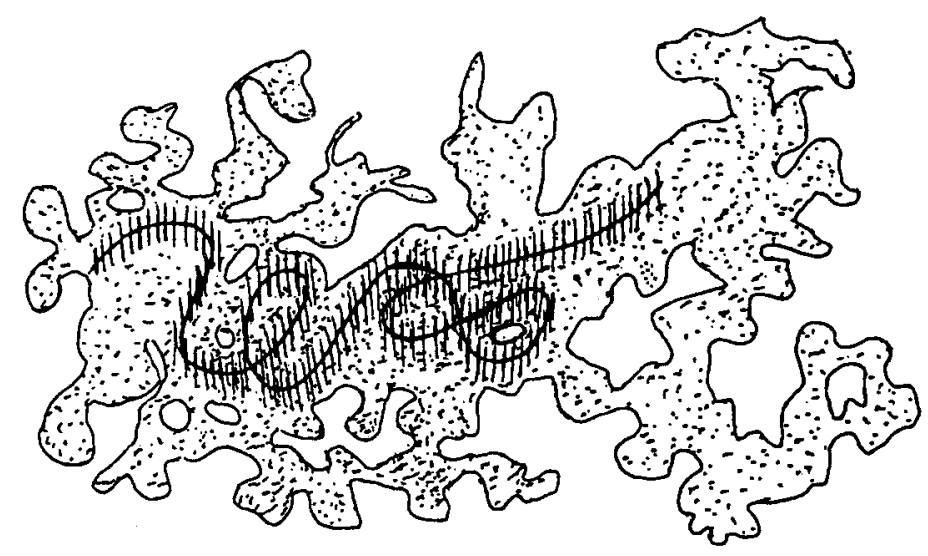

Figure 50. Schematic representation of the MC procedure for simulation of percolation and diffusion together. The shaded region represents parts of the cluster on which diffusion passes, while the dotted areas belong to the cluster but are not constructed by the MC procedure.

example, we consider the problem of diffusion on percolation clusters at criticality. Two kinds of ensemble must be taken into account (see section 3.2): the ensemble of all possible clusters, and the ensemble of the largest clusters, i.e. of only those clusters whose span is much larger than the diffusion span. For the case of all clusters, an elegant method reducing the amount of Monte Carlo work has been proposed [47]. In this method the percolation clusters and the diffusing random walker are generated by the same procedure. Each simulation consists of a random walker starting at an origin assumed to belong to the cluster. Then the nearest neighbours (NN) of the origin are chosen to be occupied or empty with probability $p$ or $1-p$ respectively ( $p$ is the probability in the percolation problem), just as in the clustergrowth method. The ant diffuses randomly to one of the occupied NN sites. This diffusion step may be carried out according to any specific algorithm, for example the myopic- or the blind-ant algorithm [388]. Now, the occupation of each of the $\mathrm{NN}$ of the latter site (to which the walker has diffused) is determined, as usual, unless, of course, its occupation has been previously determined in an earlier stage. The procedure is continued in this fashion up to the desired number of steps. The advantage of the method is that one constructs and stores only those parts of the cluster which are in the immediate neighbourhood of the walk (figure 50). Its major disadvantage is that each cluster is used for only one random walk, while overall it might be worthwhile to have several random walkers per cluster.

Another consideration that reduces the amount of numerical work for either kind of ensemble is the following. If the quantity of interest is not $d_{\mathrm{w}}$ but rather $\tilde{\zeta}$, the resistance exponent of the clusters, then the fact that $\tilde{\zeta}$ for the whole clusters is equal to $\tilde{\zeta}$ for the backbone $[82,102]$ can be used. One can simulate diffusion on the backbone alone [82], and use (2.7) to derive $\tilde{\zeta}=d_{\mathrm{w}}^{\mathrm{BB}}-d_{\mathrm{f}}^{\mathrm{BB}}$, where the superscript $\mathrm{BB}$ stands for backbone. Since $d_{\mathrm{f}}^{\mathrm{BB}}<d_{\mathrm{f}}$, diffusion is performed on a much smaller substrate. On the other hand, since $\tilde{\zeta}$ is fixed, $d_{\mathrm{w}}^{\mathrm{BB}}<d_{\mathrm{w}}$ as well. This implies that fewer random walk steps are required for a given end-to-end length of the walk. For these two reasons (the sparsity of the backbone compared with the whole cluster, and the shorter walks required) the amount of work is greatly reduced, leading to 


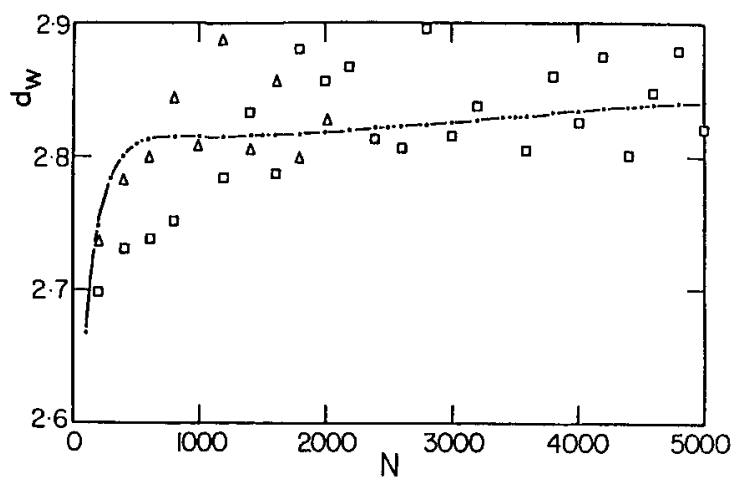

Figure 51. Graph of $d_{\mathrm{w}}$ against $N$, comparing $\operatorname{MC}$ data $(\triangle, \square)$ with exact-enumeration data (). The MC data is the same as that of figure 18.

more reliable values for $\tilde{\zeta}$ [82]. The only drawback of this method is the need for an ensemble of backbones, which is harder to simulate than the whole clusters. We hope that the recent algorithms of burning [179] for obtaining the backbones will increase the use of backbones for improving our estimates of $\tilde{\zeta}$.

\section{A.2. Exact enumeration}

This powerful technique was first used by Ben-Avraham and Havlin [47] in the early studies of anomalous diffusion on fractals and on percolation clusters, and has been reviewed by Majid et al. [83]. The method eliminates the need for a Monte Carlo simulation for the process of diffusion. Thus a Monte Carlo procedure is used at most once for generating the substrate, and all possible random walks starting from a given origin on this substrate are averaged. The exact enumeration of random walks greatly reduces the error bars, compared with Monte Carlo simulations using about the same amount of computer time (figure 51).

The key to the exact-enumeration procedure is that the probability of a random walker being at any site $i$ at some time $t$ (one can speak of time, $t$ or the $t$ th step, as convenient) is determined solely by the probabilities of being at the nearest neighbours of site $i$ at time $t-1$. The algorithm is as follows. We first store the medium on which diffusion is to take place in a matrix, keeping track of the nearest neighbours of each site. To calculate the diffusion, we have two matrices, $M_{1}\{j\}_{t}$ and $M_{2}\{j\}_{t^{\prime}}$, which store the probability distribution function $P(\mathbf{r}, t)$ of the random walker at times $t$ and $t^{\prime}$, where $\{j\}$ represents the set of all sites in the medium. Thus, given the distribution function $M_{1}\{j\}_{t}$ at time $t$, the distribution function at time $t+1$ is given by

$$
M_{2}\{j\}_{t+1}=\sum_{\operatorname{nn}\{j\}} M_{1}[n n\{j\}]_{t} W[\mathrm{nn}\{j\}, j] .
$$

Here $\mathrm{nn}\{j\}$ denotes the nearest neighbours of $\{j\}$, and $W[\mathrm{nn}\{j\}, j]$ is the probability of the walker to step from nn $\{j\}$ to $j$. The $W[\mathrm{nn}\{j\}, j]$ depend on the kind of walker (ant) in use. Having obtained $M_{2}\{j\}_{t+1}$, one can go back and calculate the distribution function at a time $t+2$ by

$$
M_{1}\{j\}_{t+2}=\sum_{\mathrm{nn}\{j\}} M_{2}[\mathrm{nn}\{j\}]_{t+1} W[\mathrm{nn}\{j\}, j] .
$$


In this fashion the probability distribution density $P\left(\mathbf{r}_{j}, t\right) \sim M\{j\}_{t}$ is obtained ( $\mathbf{r}_{j}$ is the spatial coordinate of site $j)$. Once $P(\mathbf{r}, t)$ is known, various spatial averages can be calculated (see section 2.4). The exact-enumeration procedure may easily be continued up to the time and memory limitations of the computer. The computation time increases linearly with the number of sites in the medium times the number of steps to which diffusion is performed.

Finally, we note that the exact-enumeration method can be applied to a wide range of problems involving diffusion. For example, the survival probability $\mathscr{S}(\mathbf{r}, t)$ for a diffusing particle in a random medium with traps can easily be obtained by a suitable choice of $W[\mathrm{nn}\{j\}, j]$ at the trapping sites $j$. Indeed, the method of exact enumeration has been profitably used in a wide variety of diffusion problems: diffusion on exact fractals; moments and scaling of probability densities [85]; diffusion on percolation at criticality for $d=2$ [83]; diffusion on percolation backbones [82]; diffusion on DLAs [221]; diffusion on clusters generated by random walks [85, 404]; and trapping of particles in $d=1$ [325] and $d=2$ and 3 [130]; generating DLA aggregates (S. Havlin and B. L. Trus, preprint, 1987).

\section{A.3. The 'blind ant' and the 'myopic ant'}

In the early stages of research on diffusion on percolation clusters de Gennes coined the suggestive name of 'the ant in the labyrinth'. Since then a distinction has appeared between different kinds of ants (see references [83, 398] and A. B. Harris, Y. Meir and A. Aharony, preprint, 1987), which is equivalent to different kinds of walkers having characteristic individual diffusion probabilities $W[\mathrm{nn}\{j\}, j]$ from site to site.

The blind ant does not necessarily move, but can wait at its present position with a probability equal to the number of blocked pathways divided by the coordination number of the lattice. The mypic ant, on the other hand, must move at every time step. It moves to one of the open paths lying ahead with equal probabilities for each direction. The two ants are obviously different, although computer simulations show that their walks converge to yield the same anomalous-diffusion exponents on fractals [83]. The blind ant converges more rapidly to the asymptotic regime [83, 398], possibly because of a lower probability of entering dead ends. On the other

(a)

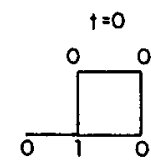

(b)

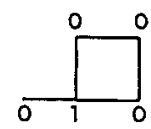

(c)

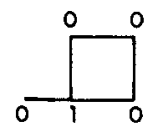

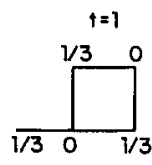
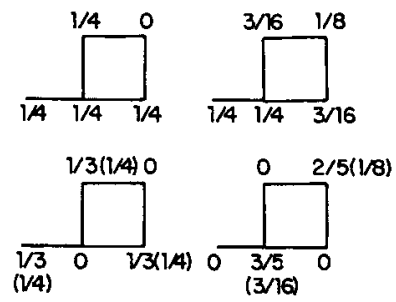

Figure 52. Time evolution of the probability distribution function for three successive instants of time for the cases of $(a)$ the myopic ant, $(b)$ the blind ant and $(c)$ the trapped ant with a mass-conserving normalization, and with no mass conservation (the case of trapping). Probabilities for the case of trapping are given in parentheses. 
hand, for some random media lacking a well-defined coordination number $z$ (for example trees with unrestricted random branching or continuum percolation of rods) the blind-ant algorithm cannot possibly be defined, and the myopic ant is most useful in such cases.

Many other algorithms are possible, some of which have important physical interpretations. For example, suppose that one enumerates the different number of ways to get to any point of the substrate in $t$ steps, starting from the origin. Dividing by the total number of possible walks, we get a third kind of ant, different from the blind and the myopic ants. However, note that we could also choose to normalize, dividing by $z^{t}$ (the total number of random walks that are possible on the underlying lattice). A moment's reflection shows that the result is the probability $\mathscr{S}(\mathbf{r}, t)$ of finding a random walker at $\mathbf{r}$ at time $t$ when the blocked sites act as perfect traps. Thus, even with the mass-conserving normalization, this third kind of ant gives a distribution that is proportional to $\mathscr{S}(\mathbf{r}, t)$.

To illustrate the different ant algorithms, we present in figure 52 the time evolution of the probability density for a myopic ant, a blind ant, and the third kind of ant - the trapped and - which has been suggested as an example.

\section{Notes added in proof}

After this review was completed we learned about additional relevant work. We list in the following the references together with the section to which they should be added.

Add to section 1, references [42] and [43]:

Numerical simulations of random walks on percolation systems are presented also in the earlier work by Hoshen and Kopelman [402].

Add to section 2:

Moments of the number of distinct sites visited by a random walker on a fractal have been studied by P. Argyrakis, L. W. Anacker and R. Kopelman (preprint, 1987).

Add to reference [137], section 2.4:

The energy spectrum of the Sierpinski gasket in a magnetic field has been studied by Banavar et al. [403].

Add to section 3:

Long-range random walk on percolation clusters was studied by Argyrakis and Kopelman [404]. The fractal behaviour of correlated random walks on percolation clusters was studied by Argyrakis and Kopelman [405]. The response function for random walks on the bond disordered lattice close to the percolation threshold is calculated in the effective medium approximation by M. E. Ernst, G. A. van Velzen and J. W. Dufty (preprint, 1987).

Add to section 3.4:

Recent numerical evaluations of the chemical distance exponents $\tilde{g}, \tilde{\nu}$ and $\tilde{\delta}$ by A. U. Neumann and S. Havlin (preprint, 1987) yield $\tilde{g}=3.3 \pm 0.1, \tilde{\nu}=0.88 \pm 0.01$ and $\tilde{\delta}=9.8 \pm 0.2$ for $d=2$ and $\tilde{g}=4.0 \pm 0.1, \tilde{\nu}=0.70 \pm 0.01$ and $\tilde{\delta}=4.2 \pm 0.1$ for $d=3$ percolation clusters. Note that these results in particular in $d=3$ are not consistent with the relation $\tilde{\delta}=(1-\tilde{\nu})^{-1}$ suggested in reference [109]. This raises a question about the validity of this relation for structures for which loops cannot be 
neglected. Manna and Chakrabarti [406] use the concept of chemical distance (minimum path) to study the dielectric breakdown in the presence of random conductors.

Add to section 3.5:

The form of $P(\mathbf{r}, t)$ for diffusion on fractals was found recently to be relevant for localization on fractals. Levy and Souillard [407] argued that the impurity quantum states on fractals are superlocalized. The wavefunction decays as $|\psi(r)| \sim \exp \left(-r^{\gamma}\right)$, $\gamma>1$. They find a relation between $\gamma$ and the exponent $u$ in $P(r, t),(2.21)$, $\gamma=u d_{\mathrm{w}}\left(d_{\mathrm{w}}+u\right)$. Assuming $u=d_{\mathrm{w}}$ as suggested in $[113,114]$ they obtained $\gamma=\left(d_{\mathrm{w}} / 2\right)>1$, i.e., superlocalization of the wave function. See also [408]. However, when using in the above formula for $a$ the result $u=\left(1-1 / d_{\mathrm{w}}\right)^{-1}$ suggested in [115] and [116] one obtains $\gamma=1$ and the wavefunction is not superlocalized. A. B. Harris and A. Aharony (preprint, 1987) prove that for averaging over all possible configurations, $|\psi(r)| \sim \exp (-r)$, i.e. $a=1$. From this and from the relation $\gamma=u d_{\mathrm{w}} /\left(d_{\mathrm{w}}+u\right)$ they argue that $u=\left(1-1 / d_{\mathrm{w}}\right)^{-1}$ for all fractals. See also related results obtained by F. Delyon, G. Deutscher, Y. E. Levy and B. Souillard (preprint, 1987).

The multifractal nature of random resistor networks was also studied by Fourcade and Tremblay [409] and by Platt and Family [410]. The voltage anomalies in random resistor networks were studied also in [411]. An indication for multifractal behaviour was found by Bunde et al. [412] for biased diffusion in percolation systems at criticality.

The voltage distribution for a two-component random mixture of conductances was studies by L. de Arcangelis and A. Coniglio (preprint, 1987). Using a scaling theory they found that an infinite hierarchy of exponent is needed to characterize systems in $D>2$, and only one exponent for $D=2$.

Add to section 3.6:

A numerical study of transport properties in two- and three-dimensional bond percolation systems has been performed by van Velzen and Ernst [413].

Recent Monte Carlo simulations of random walks on three-dimensional percolation systems were performed by R. B. Pandey, D. Stauffer and J. G. Zabolitzky (preprint, 1987). They estimate from their data that $d_{\mathrm{w}}^{\prime} \simeq 5.7 \pm 0.05$ yielding that $d_{\mathrm{w}}=4.30 \pm 0.05$ and $\tilde{\mu} \simeq 2.8 \pm 0.3$. Note that these values are appreciably larger than the previous values given in table 3 . These results deviate significantly from the Alexander-Orbach conjecture that $d_{\mathrm{s}}=2 d_{\mathrm{f}} / d_{\mathrm{w}}=4 / 3$.

Add to section 3.7:

The field theory introduced by Lubensky and Tremblay [156] for continuum percolation was re-analysed by Machta (preprint, 1987). The dynamical exponents found agree with those found by Straley [212] and Machta et al. [414] using the nodes-links-blobs model for the backbone of percolation.

Add to reference [235] section 4.3:

Maloy et al. [415].

Add to section 4.4:

For a discussion of the detailed form of $P(\mathbf{r}, t)$ in the case of CTRW in particular about the prefactor of the exponent in (4.20) see H. Weissmann, G. H. Weiss and S. Havlin (preprint, 1987). 
Add to section 5:

Haus and Kehr [416] studied the effective medium theory including weighted initial conditions, and the relation between the continuous time random walk theory and the averaged master equations. They found that the initial conditions are essential for the random trap model, yielding the previous exact results [417] for the mean square displacement, $\left\langle X^{2}\right\rangle=2 D t$, for all times.

Add to section 5.1:

Diffusion in systems with an arbitrary distribution of energy barriers and nearest neighbour hopping processes was studied by W. P. Keirstead and B. A. Huberman (preprint, 1987). Transport and spectral properties of strongly disordered chains with a power-law distribution of transition rates was studied also by Nieuwenhuizen and Ernst [418].

Add to section 5.4:

Simulations of random walks on percolation clusters with energetic disorder were studied by P. Argyrakis and R. Kopelman (preprint, 1987).

Add to section 6:

Monte Carlo simulations of biased random walks on a two-dimensional Sierpinski gasket were performed by Kim et al. [419].

Add to section 6.4:

The transport of dynamically neutral tracer flowing through a random network of tubes with percolation disorder was studied by Koplik et al. [420]. Using equation (78) of their theory [420] one obtains $d_{\mathrm{w}}^{1}=2$, compared to $d_{\mathrm{w}}^{1}=3$ obtained in (6.42) for a tracer in flow through the incipient infinite percolation cluster generated on a Cayley tree. More extensive theoretical and numerical work is needed to resolve this disagreement. A network model and effective medium approxmation for fluid flow in porous media was presented recently by R. Blumenfeld and D. J. Bergman (preprint, 1987).

Add to section 7.1:

Single and multiple random walks on random lattices were studied using excitation trapping and annihilation simulations by Kopelman et al. [421]. A scaling theory of diffusion in the presence of large traps was developed by Lyo [422]. Correlated random walks on linear chains with random traps were studied by J. K. Anlauf, K. W. Kehr and S. M. Reulein (preprint, 1987).

Add to section 7.2:

Supercomputer simulations of the elementary $\mathrm{A}+\mathrm{B} \rightarrow 0$ diffusion-limited reaction were performed under study source conditions on a Sierpinski lattice by L. W. Anacker and R. Kopelman (preprint, 1987).

Add to section 8.1:

Recently a percolation model for mixed alkali effects in solid ionic conductors was presented by Harder, Bunde and Dieterich [423] where the diffusion of hard core particles in a percolation network also was considered.

The effect of hard core interaction on diffusion in the presence of random fields and random transition rates in one-dimensional chains was studied by E. KoscienlyBunde, A. Bunde, S. Havlin and H. E. Stanley (preprint, 1987). 
Table 9. Summary of definitions, relations between exponents presented in the text, and notations of these exponents in other publications.

\begin{tabular}{|c|c|c|c|c|}
\hline Definition & $\begin{array}{l}\text { Notation in the } \\
\text { present review }\end{array}$ & \multicolumn{2}{|c|}{ In other publications } & Relations \\
\hline $\begin{array}{l}\text { Fractal } \\
\text { dimensions }\end{array}$ & $\begin{array}{l}d_{\mathrm{f}} \\
d_{\mathrm{f}}^{\mathrm{BB}} \text { (backbone) }\end{array}$ & $\begin{array}{l}D, \bar{d} \\
\bar{d}\end{array}$ & $\begin{array}{l}{[48]} \\
{[47]}\end{array}$ & \multirow[t]{2}{*}{$d_{\mathrm{f}}=d-\beta / \nu$} \\
\hline $\begin{array}{l}\text { Topological } \\
\text { exponent }\end{array}$ & $d_{1}$ & $\hat{d}$ & {$[181]$} & \\
\hline $\begin{array}{l}\text { Chemical } \\
\text { exponent }\end{array}$ & $\tilde{\nu}$ & $\begin{array}{l}1 / d_{\min } \\
Z_{1} \\
\psi_{23} \\
\nu / \nu_{\mathrm{t}}\end{array}$ & $\begin{array}{l}{[185]} \\
{[181]} \\
{[176]} \\
{[175]}\end{array}$ & $\tilde{\nu}=d_{1} / d_{\mathrm{f}}$ \\
\hline $\begin{array}{l}\text { Fracton (spectral) } \\
\text { dimension }\end{array}$ & $d_{\mathrm{s}}$ & $\begin{array}{l}\bar{d} \\
\tilde{d}\end{array}$ & {$[45]$} & $d_{\mathrm{s}}=2 d_{\mathrm{f}} / d_{\mathrm{w}}$ \\
\hline $\begin{array}{l}\text { Diffusion } \\
\text { exponents }\end{array}$ & $\begin{array}{l}d_{\mathrm{w}} \\
d_{\mathrm{w}}^{1} \\
d_{\mathrm{w}}^{\prime}\end{array}$ & $\begin{array}{l}2+\theta \\
D\end{array}$ & $\begin{array}{l}{[48]} \\
{[47]} \\
{[81]}\end{array}$ & $\begin{array}{l}d_{\mathrm{w}}=d_{\mathrm{f}}+\bar{\zeta} \\
d_{\mathrm{w}}^{1}=\tilde{\nu} d \omega \\
d_{\omega}^{\prime}=d_{\mathrm{w}} /(1-\beta / 2 \nu)\end{array}$ \\
\hline $\begin{array}{l}\text { Conductivity } \\
\text { exponent }\end{array}$ & $\tilde{\mu}=\mu / \nu$ & \multirow{2}{*}{\multicolumn{2}{|c|}{$\tilde{t}=t / \nu$}} & $\tilde{\mu}=(d-2)+\tilde{\zeta}[427]$ \\
\hline $\begin{array}{l}\text { Resistance } \\
\text { exponent }\end{array}$ & $\tilde{\zeta}=\zeta / \nu$ & & & \\
\hline
\end{tabular}

Add to section 8.2:

The fracton dimension of a family of deterministic fractals has recently been studied by Borgan et al. [424].

Add to section 8.3:

D. Markovic, S. Milosevic and H. E. Stanley (preprint, 1987), studied SAWs on percolation systems. Within the framework of the position-space renormalization group (PSRG) method they find that the critical exponent $\nu$ of the mean end-to-end distance of SAW on a two-dimensional random network is equal to the critical exponent of SAW on the ordinary square lattice. An exact solution of a kinetic selfavoiding walk on the Sierpinski gasket was presented by Bradley [425].

Add to section 8.4:

T. Odagaki (preprint, 1987) has studied the termite and ant diffusions in the $d$-dimensional lattice trapping model. Dekeyser et al. [426] have studied the static and dynamic fractal properties of random walks with intersections. The fracton dimensionality of random superconducting-normal networks has been numerically calculated by A. R. Day, W. Xia and M. L. Thorpe (preprint, 1987).

For the convenience of the reader we summarize in table 9 the definitions of the different exponents, important relations between the exponents presented in the text, and the notation used in other publications. 


\section{References}

[1] Pynn, R., and SkJeltorp, A., (eds), 1985, Scaling Phenomena in Disordered Systems, NATO ASI Series B, Vol. 133 (New York: Plenum).

[2] Engelman, R., and JAeger, Z., (eds), 1986, Fragmentation Form and Flow in Fractured Media (Bristol: IPS).

[3] Haus, J. W., and Kehr, K. W., 1987, Phys Rep., 150, 263.

[4] Alexander, S., Bernascon, J., Schneider, W. R., and Orbach, R., 1981, Rev. mod. Phys., 53, 175.

[5] Burridge, R., Childress, S., and Papanicolau, G., (eds), 1982, Proc. Conf. on Macroscopic Properties of Disordered Media (New York: Springer Verlag).

[6] Proc. Second Bar-Ilan Conf. on Physics of Disordered Systems, 1987, Phil. Mag. B, 56.

[7] Boccara, N., and Daoud, M., (eds), 1985, Physics of finely divided matter. Proc. Winter School, Les Houches, 1985 (Heidelberg: Springer-Verlag).

[8] Family, F., and Landau, D. P., (eds), 1984, Proc. Int. Topical Conf. Kinetics of Aggregation and Gelation, Athens, GA (Amsterdam: North-Holland).

[9] Stanley, H. E., and Ostrowsky, N., (eds), 1986, On Growth and Form (Boston, MA: Nartinus Nijhoff).

[10] De Gennes, P. G., 1979, Scaling Concepts in Polymer Physics (Ithaca, NY: Cornell University Press).

[11] Herrmann, H. J., 1986, Phys. Rep., 136, 153.

[12] Blumen, A., Klafter, J., and Zumofen, G., 1986, Optical Spectroscopy of Glasses, edited by I. Zschokke (Dordrecht: Reidel), p. 199.

[13] Bunde, A., 1986, Adv. Solid St. Phys., 26, 113.

[14] Weiss, G. H., and Rubin, R. J., 1983, Adv. chem. Phys., 52, 363.

[15] Shlesinger, M. F., and West, B. J., (eds), 1984, Random Walks and their Applications in the Physical and Biological Sciences, AIP Conf. Proc. 109 (New York: AIP).

[16] Kir KPAtrick, S., 1979, Le Houches Summer School on Ill Condensed Matter, edited by R. Maynard and G. Toulouse (Amsterdam: North-Holland).

[17] Domb, C., Stoll, E., and Schneider, T., 1980, Contemp. Phys., 21, 577.

[18] Stauffer, D., 1985, Introduction to Percolation Theory (London: Taylor \& Francis).

[19] Essam, J. W., 1980, Rep. Prog. Phys., 43, 843.

[20] Deutscher, G., Zallen, R., and Adler, J., (eds), 1983, Percolation Structures Processes, Ann. Isr. phys. Soc., Vol. 5 (Bristol: Hilger).

[21] Kesten, H., 1982, Percolation Theory for Mathematicians (Boston, MA: Birkauser).

[22] Aharony, A., 1986, S.K. Ma Memorial Book (Singapore: World Scientific).

[23] Mandelbrot, B. B., 1977, Fractals: Form, Chance and Dimension (San Francisco: Freeman).

[24] Mandelbrot, B. B., 1982, The Fractal Geometry of Nature (San Francisco: Freeman).

[25] Pietronero, L., and Tosatti, E., (eds), 1986, Fractals in Physics (Amsterdam: NorthHolland).

[26] Proc. Symp. on Fractals in the Physical Scinces, 1984, J. statist. Phys., 36.

[27] Stanley, H. E., and Meakin, P., 1987, Nature (to be published).

[28] Stanley, H. E., 1987, Introduction to Fractal and Multifractal Phenomena (Oxford University Press).

[29] Guyon, E., Hulin, J. P., and Lenormand, R., 1984, Ann. Mines, 17, 40.

[30] Nittmann, J., Daccord, G., and Stanley, H. E., 1985, Nature, 314, 141; Nittmann, J., and Stanley, H. E., 1986, Nature, 321, 663.

[31] Salomon, M. B., and Yeshurun, Y., 1988 (to be published).

[32] Uemura, Y. and J., and Birgeneau, R. J., 1986, Phys. Rev. Lett., 57, 1947.

[33] Courtens, E., Pelous, J., Phalippou, J., Vacher, R., and Woignier, T., 1987, Phys. Rev. Lett., 58, 128.

[34] Avogadro, A., Aldrovandi, S., and Borsa, F., 1986, Phys. Rev. B, 33, 5637.

[35] Grinstein, G., and Fernandez, J. F., 1984, Phys. Rev. B, 29, 6389; Birgenau, R. J., Shirane, G., and Yoshizawa, H., 1985, Phys. Rev. Lett., 54, 2147.

[36] Levitt, N., 1982, Ann. Rev. Biophys. Bioengng, 11, 251.

[37] Bernascon, J., Beyeler, J. U., Strassler, S., and Alexander, S., 1979, Phys. Rev. Lett., 42, 819.

[38] Kapitulnik, A., and Deutscher, G., 1982, Phys. Rev. Lett., 49, 1944. 
[39] Voss, R., Leibowitz, R., and Alessandrini, E., 1982, Phys. Rev. Lett., 49, 1441.

[40] Palevski, A., and Deutscher, G., 1984, J. Phys. A, 17, L895.

[41] Kopelman, R., 1986, J. statist. Phys., 42, 185.

[42] De Gennes, P. G., 1976, La Recherche, 7, 919.

[43] Mitescu, C. D., Ottavi, H., and Rossenq, J., 1979, Electrical Transport and Optical Properties of Inhomogeneous Media, Ohio State University, 1977, edited by J. C. Garland and D. B. Tanner, AIP Conf. Proc. 40 (New York: AIP), p. 377.

[44] Gefen, Y., Aharony, A., Mandelbrot, B. B., and Kirkpatrick, S., 1981, Phys. Rev. Lett., 47, 1771.

[45] Alexander, S., and Orbach, R., 1982, J. Phys. Lett., Paris, 43, L625.

[46] Webman, I., 1981, Phys. Rev. Lett., 47, 1497.

[47] Ben-Avraham, D., and Havlin, S., 1982, J. Phys. A, 15, L691; Havlin, S., BenAvraham, D., and Sompolinsky, H., 1983, Phys. Rev. A, 27, 1730.

[48] Gefen, Y., Aharony, A., and Alexander, S., 1983, Phys. Rev. Lett., 50, 77.

[49] Rammal, R., and Toulouse, G., 1983, J. Phys. Lett., Paris, 44, L13.

[50] Meakin, P., and Stanley, H. E., 1983, Phys. Rev. Lett., 51, 1457.

[51] Wilke, S., Gefen, Y., Iklovic, V., Aharony, A., and Stauffer, D., 1984, J. Phys. A, 17, 647.

[52] Havlin, S., Duordjevic, Z. V., Majid, I., Stanley, H. E., and Weiss, G. H., 1984, Phys. Rev. Lett., 53, 178.

[53] Ben-Avraham, D., and Havlin, S., 1983, J. Phys. A, 16, L559.

[54] Weiss, G. H., 1970, Sep. Sci., 5, 51.

[55] Montroll, E. W., and Weiss, G. H., J. math. Phys., 6, 167.

[56] Gefen, Y., Mandelbrot, B. B., and Aharony, A., 1980, Phys. Rev. Lett., 45, 855.

[57] Havlin, S., and Nossal, R., 1984, J. Phys. A, 17, L427.

[58] Weitz, D. A., Lin, M. Y., Huang, J. S., Witten, T. A., Sinha, S. K., Gethner, J. S., and Ball, R. C., 1985, Scaling Phenomena in Disordered Systems, Nato, ASI Series B, Vol. 133 (New York: Plenum), p. 171.

[59] Schaefer, D. W., and Keefer, K. D., 1984, Phys. Rev. Lett., 53, 1383; Proc. Int. Topical Conf. on Kinetics of Aggregation and Gelation, Athens, GA, edited by F. Family and D. P. Landau (Amsterdam: North-Holland), p. 39.

[60] Kuems, K., and Freltoft, T., 1985, Scaling Phenomena in Disordered Systems, NATO ASI Series, edited by R. Pynn and A. Skjeltorp (New York: Plenum), p. 133.

[61] Weitz, D. A., and Oliveria, M., 1984, Phys. Rev. Lett., 53, 1433.

[62] Schaeffer, D. W., Martin, J. E., Wiltzius, P., and Cannell, D. S., 1984, Phys. Rev. Lett., 52, 2371.

[63] Witten, T. A., and Sander, L. M., 1981, Phys. Rev. Lett., 47, 1400.

[64] Niemeyer, L., Pietronero, L., and Weismann, A. J., 1984, Phys. Rev. Lett., 52, 1033.

[65] Langer, J. S., 1986, Physica A, 140, 44.

[66] Sawada, Y., 1986, Physica A, 140, 134.

[67] Stapleton, H. J., Allen, J. P., Flynn, C. P., Stinson, D. G., and Kurtz, S. R., 1980, Phys. Rev. Lett., 45, 1456.

[68] Havlin, S., and Ben-Avraham, D., 1982, Phys. Rev. A, 26, 1728.

[69] Cates, M. E., 1984, Phys. Rev. Lett., 53, 926.

[70] Alexander, S., Laermans, C., Orbach, R., and Rosenberg, H. M., 1983, Phys. Rev. B, 28, 4615.

[71] OrR, W. J., 1947, Trans. Faraday Soc., 43, 12.

[72] Chandrasekhar, S., 1943, Rev. mod. Phys., 15, 1.

[73] Gefen, Y., Aharony, A., and Mandelbrot, B. B., 1983, J. Phys. A, 16, 1267; Gefen, Y., Aharony, A., Shapir, Y., and Mandelbrot, B. B., 1984, J. Phys. A, 17, 435.

[74] Havlin, S., and Ben-Avraham, D., 1982, J. Phys. A, 15, L311.

[75] Gould, H., and Kohin, R. P., 1984, J. Phys. A, 17, L159.

[76] Given, J. A., and Mandelbrot, B. B., 1983, J. Phys. A, 16, L565.

[77] Havlin, S., 1985, Phys. Rev. Lett., 55, 130.

[78] Cates, M. E., 1985, Phys. Rev. Lett., 55, 131. 
[79] Kirk Patrick, S., 1979. Inhomogeneous Superconductors, edited by D. U. Gubser, T. L. Francarilla, J. R., Leibowitz and S. A. Wolf, AIP Conf. Proc. 58 (New York: American Institute of Physics).

[80] Havlin, S., and Ben-Avraham, D., 1983, J. Phys. A, 16, L483.

[81] Pandey, R. B., and Stauffer, D., Phys. Rev. Lett., 51, 527.

[82] Hong, D. C., Havlin, S., Hermann, H. J., and Stanley, H. E., 1984, Phys. Rev. B, 30, 4083.

[83] Majid, I., Ben-Avraham, D., Havlin, S., and Stanley, H. E., 1984, Phys. Rev. B, 30, 1626.

[84] Meakin, P., Majid, I., Havlin, S., and Stanley, H. E., 1984, J. Phys. A, 17, L975.

[85] Havlin, S., Weiss, G. H., Ben-Avraham, D., and Movshovitz, D., 1984, J. Phys. A, 17, L849.

[86] Havlin, S., Nossal, R., Trus, B., and Weiss, G. H., 1985, Phys. Rev. B, 31, 7497.

[87] Dhar, D., and Ramaswamy, R., 1985, Phys. Rev. Lett., 54, 1346.

[88] Derrida, B., and Vannimenus, J., 1982, J. Phys. A, 15, L557.

[89] Derrida, B., Zabolitzky, J. G., Vannimenus, J., and Stauffer, D., 1984, J. statist. Phys., 36, 31.

[90] Zabolitzky, J. G., 1984, Phys. Rev. B, 30, 4077.

[91] Kittel, C., 1971, Introduction to Solid State Physics (New York: Wiley).

[92] Alexander, S., Bernasconi, J., Schneider, W. R., and Orbach, R., 1978, J. Phys., Paris, C, 6, 706 .

[93] Leyvraz, F., and Stanley, H. E., 1983, Phys. Rev. Lett., 51, 2048.

[94] Harris, A. B., Kim, S., and Lubensky, T. C., 1984, Phys. Rev. Lett., 53, 743.

[95] Aharony, A., and Stauffer, D., 1984, Phys. Rev. Lett., 52, 2368.

[96] Havlin, S., 1984, Phys. Rev. Lett., 53, 1705.

[97] Daoud, M., 1983, J. Phys. Lett., Paris, 44, 1925.

[98] Herrmann, H. J., Derrida, B., and Vannimenus, J., 1984, Phys. Rev. B, 30, 4080.

[99] Lobb, C. J., and Frank, D. J., 1984, Phys. Rev. B, 30, 4090.

[100] Rammal, R., Angles d’Auriac, J. C., and Benoit, A., 1984, Phys. Rev. B, 30, 4087.

[101] Stanley, H. E., Majid, I., Margolina, A., and Bunde, A., 1984, Phys. Rev. Lett., 53, 1706.

[102] Stanley, H. E., and Coniglio, A., 1984, Phys. Rev. B, 29, 522.

[103] Alexander, S., Percolation Structures and Processes, edited by G. Deutsche, R. Zallan and J. Adler, Ann. Israel Phys. Soc., Vol. 5 (Bristol, UK: Adam Hilger).

[104] Essam, J. W., and Bhatti, F. M., 1985, J. Phys. A, 18, 3577.

[105] Aharony, A., Alexander, S., Entin-Wohlman, O., and Orbach, R., 1987, Phys. Rev. Lett., 58, 132.

[106] De Gennes, P. G., 1976, J. Phys. Lett., Paris, 37, L1.

[107] Skal, A. S., and ShklovskiI, B. I., 1974, Fiz. Tekh. Polyprovodn, 8, 1586; 1975, Soviet Phys. Semicond., 8, 1029.

[108] Pfister, G., and Scher, H., 1978, Adv. Phys., 27, 747.

[109] Havlin, S., Trus, B. L., Weiss, G. H., and Ben-Avraham, D., 1985, J. Phys. A, 18, L247.

[110] Domb, C., 1969, Adv. chem. Phys., 15, 229; Fisher, M. E., 1966, J. chem. Phys., 44, 616; Fisher, M. E., and Burford, R. J., 1967, Phys. Rev., 156, 583; McKenzie, D. S., 1976, Phys. Rep., 27, 2; McKenzie, D. S., and Moore, M. A., 1971, J. Phys. A, 4, L82.

[111] O’Shaughnessy, B., and Procaccia, I., 1985, Phys. Rev. Lett., 54, 455; 1985, Phys. Rev., 32, 3073.

[112] Banavar, J. R., and Willemson, J., 1984, Phys. Rev. B, 30, 6778.

[113] Guyer, R. A., 1984, Phys. Rev. A, 29, 2751.

[114] Havlin, S., Movshovitz, D., Trus, B. L., and Weiss, G. H., 1985, J. Phys. A, 18, L719.

[115] Halsey, T. C., Jensen, M. H., Kadanoff, L. P., Procaccia, I., and Shraiman, B. I., 1986, Phys. Rev. A, 33, 1141. 
[116] de Arcangelis, L., Redner, S., and Coniglio, A., 1985, Phys. Rev. B, 31, 4725; 1986, Phys. Rev. B, 34, 4656.

[117] Meakin, P., Stanley, H. E., Coniglio, A., and Witten, T. A., 1985, Phys. Rev. A, 32, 2364; Meakin, P., Coniglio, A., Stanley, H. E., and Witten, T. A., 1986, Phys. Rev. A, 34, 3325.

[118] Halsey, T. L., Meakin, P., and Procaccia, I., 1986, Phys. Rev. Lett., 56, 854.

[119] Amitrano, C., Coniglio, A., and di Liberto, F., 1986, Phys. Rev. Lett., 57, 1016.

[120] Mandelbrot, B. B., 1978, Proc. 13th IUPAP Conf. on Statistical Physics edited by E. Cabib, C. G. Kuper and I. Reiss (Bristol, UK: Adam Hilger).

[121] Hentschel, H. G. E., and Procaccia, I., 1983, Physica D, 8, 435.

[122] Blumenfeld, R., Meir, Y., Harris, A. B., and Aharony, A., 1986, J. Phys. A, 19, L91; Meir, Y., Blumenfeld, R., Aharony, A., and Harris, A. B., 1986, Phys. Rev. $\mathrm{B}, 34,3424$.

[123] Ramel, R., and Tremblay, A. M. S., 1987, Phys. Rev. Lett., 58, 415.

[124] Khanta, M., and Yeomans, J. M., 1987, J. Phys. A, 20, L325.

[125] Toulous, G., 1974, Nuovo Cim. B, 23, 234; Harris, A. B., Lubensky, T. C., Holcomb, W. K., and Dasgupta, C., 1975, Phys. Rev. Lett., 35, 327, 1397(E).

[126] Donsker, N. D., and Varadhan, S. R. S., 1979, Commun. pure appl. Math., 32, 721.

[127] Grassberger, P., and Proccacia, I., 1982, J. chem. Phys., 77, 6281.

[128] Toussaint, D., and Wilczek, F., 1983, J. chem. Phys., 78, 2642.

[129] Redner, S., and Kang, K., 1983, Phys. Rev. Lett., 81, 1729.

[130] Havlin, S., Dishon, M., Kiefer, J. E., and Weiss, G. H., 1984, Phys. Rev. Lett., 53, 407.

[131] Weiss, G. H., and Havlin, S., 1984, J. statist. Phys., 37, 17.

[132] Bergman, D., and Kantor, Y., 1984, Phys. Rev. Lett., 53, 511.

[133] Zumofen, G., Blumen, A., and Klafter, J., 1984, J. Phys. A, 17, L479.

[134] Straley, J. P., 1980, J. Phys. C, 13, 2991.

[135] Anacker, L. W., and Kopelman, R., 1987, Phys. Rev. Lett., 58, 289.

[136] Robillard, S., and Tremblay, A. M. S., 1986, J. Phys. A, 19, 2171.

[137] Domany, E., Alexander, S., Bensimon, D., and Kadanoff, L. P., 1983, Phys. Rev. $\mathrm{B}, \mathbf{2 8}, 3110$.

[138] Rammal, R., and Toulouse, G., 1982. Phys. Rev. Lett., 49, 1194.

[139] Broadbent, S. R., and Hammersley, J. M., 1987, Proc. Camb. phil. Soc., 53, 629.

[140] Domb, C., and Sykes, M. F., 1961, Phys. Rev., 122, 77.

[141] Stauffer, D., 1979, Phys. Rep., 54, 1.

[142] Bunde, A., Dieterich, W., and Roman, E., 1985, Phys. Rev. Lett., 55, 5; Roman, H. E., Bunde, A., and Dieterich, W., 1986, Phys. Rev. B, 34, 3439.

[143] Mackay, G., and Jan, N., 1984, J. Phys. A, 17, L757.

[144] Grassberger, P., 1983, Math. Biosci., 63, 157.

[145] Bunde, A., Herrmann, H., Margolina, A., and Stanley, H. E., 1985, Phys. Rev. Lett., 55, 653.

[146] Ziff, R. M., and Sapoval, B., 1987, J. Phys. A, 19, L1169.

[147] Den Niss, M. P. M., 1979, J. Phys. A, 12, 1857.

[148] Heermann, D. W., and Stauffer, D., 1981, Z. Phys. B, 44, 339.

[149] Gaunt, D. S., and Sykes, M. F., 1983, J. Phys. A, 16, 1783.

[150] Coniglio, A., 1982, J. Phys. A, 15, 3829.

[151] Mandelbrot, B. B., and Given, J. A., 1984, Phys. Rev. Lett., 52, 1853.

[152] Argyrakis, P., and Kopelman, R., 1984, Phys. Rev. B, 29, 511.

[153] Halperin, B. I., Feng, S., and Sen, P. N., 1985, Phys. Rev. Lett., 54, 2391.

[154] Elan, W. T., Kerstein, A. R., and Rehr, J. J., 1984, Phys. Rev. Lett., 52, 1516.

[155] Sen, P. N., Roberts, J. N., and Halperin, B. I., 1985, Phys. Rev. B, 32, 3306.

[156] Lubensky, T. C., and Tremblay, A. M. J., 1986, Phys. Rev. B, 34, 3408.

[157] Machta, J., Guyer, R. A., and Moore, S. M., 1986, Phys. Rev. B, 33, 4818.

[158] Bunde, A., Harder, H., and Havlin, S., 1986, Phys. Rev. B, 34, 3540.

[159] Weiss, G. H., and Havlin, S., 1987, Phys. Rev. B, 36, 807.

[160] Feng, S., Halperin, B. I., and Sen, P. N., 1987, Phys. Rev. B, 35, 197.

[161] Frisch, H. L., and Harmmersley, J. M., 1963, J. Soc. ind. Appl. Math., 11, 894.

[162] Balberg, I., 1987, Phil. Mag. B, 56, 995. 
[163] Domb, C., 1986, Fragmentation, Form and Flow in Fractured Media, edited by R. Englman and Z. Jaeger (Bristol, UK: Hilger), p. 102.

[164] Kapitulnik, A., Aharony, A., Deutscher, G., and Stauffer, D., 1983, J. Phys. A, 16, L269.

[165] Domb, C., 1976, J. Phys. A, 9, L141.

[166] Sapoval, B., Rosso, M., Gouyet, J. F., and Colonna, J. F., 1986, Solid St. Ionics $18 / 19,21$.

[167] Stanley, H. E., 1977, J. Phys. A, 10, L211.

[168] Harrison, R. J., Bishop, G. H., and Quinn, G. D., 1978, J. statist. Phys., 19, 53.

[169] Kopelman, R., 1976, Topics in Applied Physics, Vol. 15, edited by F. K. Fong (Heidelberg: Springer-Verlag).

[170] Viscek, T., 1981, Z. Phys. B, 45, 153.

[171] Derrida, B., Orbach, R., and Yu, K. W., 1984, Phys. Rev. B, 29, 6645.

[172] Orbach, R., 1986, Science, 231, 814 and references therein.

[173] Aharony, A., Alexander, S., Entin-Wohlman, O., and Orbach, R., 1985, Phys. Rev. B, 31, 2565.

[174] Middlemiss, K. M., Whittington, S. G., and Gaunt, D. C., 1980, J. Phys. A, 13, 1835.

[175] Alexandrowicz, Z., 1980, Phys. Lett. A, 80, 284.

[176] Pike, R., and Stanley, H. E., 1981, J. Phys. A, 14, L169.

[177] Hong, D. C., and Stanley, H. E., 1983, J. Phys. A, 16, 1475; 1983, Ibid., 16, L525.

[178] Vannimenus, J., Nadal, J. P., and Martin, H., 1984, J. Phys. A, 17, L351.

[179] Herrmann, H. J., Hong, D. C., and Stanley, H. E., 1984, J. Phys. A, 17, L261.

[180] Ritzenberg, A. L., and Cohen, R. I., 1984, Phys. Rev. B, 30, 4036.

[181] Grassber ger, P., 1986, Math. Biosci., 62, 157; 1986, J. Phys. A, 19, 1681.

[182] Witten, T. A., and Kantor, Y., 1984, Phys. Rev. B, 30, 4093.

[183] Havlin, S., 1984, Kinetics of Aggregation and Gelation, edited by F. Family and D. P. Landau (Amsterdam: Elsevier).

[184] Leath, P. L., 1976, Phys. Rev. B, 14, 5046.

[185] Stanley, H. E., 1984, J. statist. Phys., 36, 843.

[186] Grassberger, P., 1985, J. Phys. A, 18, L215.

[187] Edwards, B. F., and Kerstein, A. R., 1985, J. Phys. A, 18, L1081.

[188] Margolina, A., 1985, J. Phys. A, 18, L651.

[189] Webman, I., 1984, Proc. Int. Topical Conf. on Kinetics of Aggregation and Gelation, Athens, GA, edited by F. Family and D. P. Landau (Amsterdam: North-Holland); Kantor, Y., and Webman, I., 1984, Phys. Rev. Lett., 52.

[190] Family, F., and Coniglio, A., 1984, J. Phys. A, 17, L285.

[191] Roux, S., 1985, J. Phys. A, 18, L395.

[192] Issacson, J., and Lubensky, T. C., 1980, J. Phys. Lett., Paris, 41, L469.

[193] Larsson, T. A., 1987, J. Phys. A, 20, L291.

[194] Cardy, J. L., and Grassberger, P., 1985, J. Phys. A, 18, L267.

[195] Barma, M., 1985, J. Phys. A, 18, L277.

[196] Rammal, R., Tannous, C., and Tremblay, A. M. S., 1985, Phys. Rev. A, 31, 1662.

[197] Castellani, C., and Peliti, L., 1986, J. Phys. A, 19, L429.

[198] Blumenfeld, R., and Aharony, A., 1985, J. Phys. A, 18, L443.

[199] Mandelbrot, B. B., 1974, J. Fluid Mech., 62, 331.

[200] Bug, A. L. R., Grest, G. S., Cohen, M. H., and Webman, I., 1986, J. Phys. A, 19, L323.

[201] Bernasconi, J., 1978, Phys. Rev. B, 18, 2185.

[202] Herrmann, H., and Stanley, H. E., 1984, Phys. Rev. Lett., 53, 1121.

[203] Voss, R. F., 1984, J. Phys. A, 16, 1475.

[204] Priest, R. G., and Lubensky, T. C., 1976, Phys. Rev. B, 13, 4159.

[205] Амit, D. J., 1976, J. Phys. A, 9, 1441.

[206] De Alcantar Bonfim, O. F., Kirkhaus, J. E., and McKane, A. J., 1981, J. Phys. A, 14, 2391.

[207] Adler, J., 1985, J. Phys. A, 18, 307.

[208] Mitescu, C. D., and Musoff, M. J., 1981, J. Phys. Lett., Paris, 44, L679.

[209] Gawlinski, E. T., and Stanley, H. E., 1981, J. Phys. A, 14, L291. 
[210] Kogut, P. M., and Straley, J. P., 1979, J. Phys. C, 12, 2151.

[211] Ben-Mizrahi, A., and Bergman, D. J., 1981, J. Phys. C, 14, 909.

[212] Straley, J. P., 1982, J. Phys. C, 15, 2333, 2343.

[213] Harris, A. B., 1987, Phil. Mag. B, 56, 835.

[214] Benguigui, L., 1986, Phys. Rev. B, 34, 8177.

[215] Harris, A. B., and Lubensky, T. C., 1984, J. Phys. A, 17, L609.

[216] Wang, J., and Lubensky, T. C., 1986, Phys. Rev. B, 33, 4998.

[217] Jan, N., Hong, D. C., and Stanley, H. E., 1985, J. Phys. A, 18, L935.

[218] Zimm, B. H., and Stockmayer, W. H., 1949, J. chem. Phys., 17, 1301.

[219] Lubensky, T. C., and Isaacson, J., 1981, Phys. Rev. Lett., 46, 871.

[220] Havlin, S., Trus, B., and Stanley, H. E., 1984, Phys. Rev. Lett., 53, 1288.

[221] Meakin, P., and Stanley, H. E., 1983, Phys. Rev. Lett., 51, 1457.

[222] Havlin, S., Nossal, R., and Trus, B., 1985, Phys. Rev. A, 32, 3829.

[223] Parisi, G., and Sourlas, N., 1981, Phys. Rev. Lett., 46, 871.

[224] Dhar, D. H., 1983, Phys. Rev. Lett., 51, 853.

[225] Martin, J., 1972, Phase Transitions and Critical Phenomena, Vol. 3, edited by C. Domb and M. S. Green (New York: Academic Press); Redner, S., 1982, J. statist. Phys., 29, 309.

[226] Duordjevic, Z. V., Havlin, S., Stanley, H. E., and Weiss, G. H., 1984, Phys. Rev. B, 30, 478.

[227] Hohenberg, P. C., and Halperin, B. I., 1977, Rev. mod. Phys., 49, 435.

[228] Meakin, P., 1983, Phys. Rev. A, 27, 604.

[229] Meakin, P., 1983, Phys. Rev. A, 27, 1495.

[230] Witten, T. A., and Cates, M. E., 1986, Science, 232, 1607.

[231] Elam, W. T., Wolf, S. A., Sprayne, J., Van Vetchten, D., Barz, G. L., Jr, and Meakin, P., 1985, Phys. Rev. Lett., 54, 701.

[232] Brady, R. M., and BAll, R. C., 1984, Nature, 309, 225.

[233] Witten, T. A., and Sander, L. M., 1983, Phys. Rev. B, 27, 5686.

[234] Niemeyer, L., and PinneKamp, F., 1982, Proc. Int. Symp. on Gaseous Dielectric, Knoxville, TN (New York: Pergamon), p. 379.

[235] Patterson, L., 1984, Phys. Rev. Lett., 52, 1621.

[236] Meakin, P., 1983, Phys. Rev. Lett., 51, 1119.

[237] Kolb, M., Botet, R., and Jullien, R., 1983, Phys. Rev. Lett., 51, 1123.

[238] Ball, R. C., and Witten T. A., 1984, Phys. Rev. A, 29, 2966.

[239] Weiss, G., and Havlin, S., 1986, Physica A, 134, 474.

[240] Barma, M., and Dhar, D., 1983, J. Phys. C, 16, 1451.

[241] White, S. R., and Barma, M., 1984, J. Phys. A, 17, 2995.

[242] Goldhirsch, I., and Gefen, Y., 1987, Phys. Rev. A, 35, 1317.

[243] Shlesinger, M. F., 1974, J. statist. Phys., 10, 421.

[244] Blumen, A., Klafter, J., White, B. S., and Zumofen, G., 1984, Phys. Rev. Lett., 53, 1301.

[245] Ball, R., Havlin, S., and Weiss, G. H., 1987, J. Phys. A, 20, 4055.

[246] Ohtsuki, T., and Keyes, T., 1984, Phys. Lett. A, 105, 273; 1984, Phys. Rev. Lett., 52, 1177.

[247] Havlin, S., Nossal, R., Trus, B., and Weiss, G. H., 1984, J. Phys. A, 17, 1957.

[248] Havlin, S., 1986, Fractals in Physics, edited by L. Pietronero and E. Tosatti (Amsterdam: Elsevier).

[249] Family, F., 1982, J. Phys. A, 15, L583.

[250] Halperin, V., 1981, J. Phys. C, 14, 3195; Halperin, V., and Sonnenschein, M., 1981, J. Phys. C, 14, 3209.

[251] Halperin, V., and Shatz, S., 1984, Chem. Phys., 91, 233.

[252] Dyson, F. J., 1953, Phys. Rev., 92, 1331.

[253] Schmidt, J., 1957, Phys. Rev., 105, 425.

[254] Domb, C., 1963, Proc. R. Soc., Lond. A, 276, 418.

[255] Halperin, B. I., 1965, Phys. Rev. A, 139, 104; 1966, Adv. chem. Phys., 13, 123.

[256] Bernasconi, J., Alexander, S., and Orbach, R., 1987, Phys. Rev. Lett., 41, 185.

[257] Alexander, S., 1981, Phys. Rev. B, 23, 2951.

[258] Stephen, M. J., and Katiotis, R., 1982, Phys. Rev. B, 26, 2917. 
[259] Havlin, S., Bunde, A., Stanley, H. E., and Movshovitz, D., 1986, J. Phys. A, 19, L693.

[260] Bunde, A., Havlin, S., Stanley, H. E., Trus, B., and Weiss, G. H., 1986, Phys. Rev. B, 34, 8129.

[261] Sompolinsky, H., 1981, Phys. Rev. Lett., 47, 935.

[262] Teitel, S., and Domany, E., 1985, Phys. Rev. Lett., 5, 2176.

[263] Rammal, R., Toulouse, G., and Virasoro, M. A., 1986, Rev. mod. Phys., 58, 765.

[264] Pandey, R. B., Stauffer, D., Margolina, A., and Zabolitsky, J. G., 1986, J. statist. Phys., 34, 427.

[265] Machta, J., 1981, Phys. Rev. B, 24, 5260.

[266] Zwanzig, R., 1982, J. statist. Phys., 28, 127.

[267] Kundu, K., and Phillips, P., 1987, Phys. Rev. A, 35, 857; Kundu, K., Parris, P. E., and Phillips, P., 1987, Phys. Rev. A, 35, 3468.

[268] Bouchaud, J. P., Comtet, A., Georges, A., and Le Doussal, P., 1987, Europhys. Lett., 3, 635.

[269] Dentener, P. J. H., and Ernst, M. H., 1984, Phys. Rev. B, 29, 1755.

[270] Havlin, S., Weissman, H., and Matan, O., 1987 (to be published).

[271] Huberman, B. A., and Kerszberg, M., 1985, J. Phys. A, 18, L331.

[272] Kutasov, D., Aharoni, A., Domany, E., and Kinzel, W., 1986, Phys. Rev. Lett., 56, 2229.

[273] Grossmann, S., Wegner, F., and Hoffmann, K. H., 1985, J. Phys. Lett., Paris, 46, L575.

[274] Hablin, S., and Weissman, H., 1986, J. Phys. A, 19, L1021.

[275] Blumen, A., Zumofen, G., and Klafter, J., 1986, J. Phys. A, 19, L861.

[276] Havlin, S., Bunde, A., Glaser, Y., and Stanley, H. E., 1986, Phys. Rev. A, 34.

[277] Havlin, S., and Weissman, H., 1987, Phys. Rev. B (in press).

[278] SinaI, YA., 1982, Proceedings of the Berlin Conference on Mathematical Problems in Theoretical Physics, edited by R. Schrader, R. Seiler and D. A. Uhlenbroch (Berlin: Springer), p. 12; 1982, Theor. Prob. Appl., 27, 256.

[279] Pandey, R. B., 1986, J. Phys. A, 19, 3925.

[280] Winter, C. L., Newman, C. M., and Neuman, S. P., 1984, SiaM J. appl. Math., 44, 411.

[281] Derrida, B., and Pomeau, Y., 1982, Phys. Rev. Lett., 48, 627.

[282] Havlin, S., Bunde, A., Weissman, H., and Aharony, A., 1987, Phys. Rev. B, 35, 397.

[283] Havlin, S., and Webman, I., 1987 (to be published).

[284] Havlin, S., Trus, B. L., and Weiss, G. H., 1986, J. Phys. A, 19, L817.

[285] Machta, J., 1985, J. Phys. A, 18, L531.

[286] Scher, H., and Lax, M., 1973, Phys. Rev. B, 7, 4491.

[287] Scher, H., and Montroll, E., 1975, Phys. Rev. B, 12, 2455.

[288] Klafter, J., and Silbey, R., 1980, Phys. Rev. Lett., 44, 55; 1980, J. chem. Phys., 72, 843.

[289] Kivelson, S., 1980, Phys. Rev. B, 21, 5755.

[290] Harder, H., Bunde, A., and Havlin, S., 1987, Phys. Rev. A, (in press).

[291] Havlin, S., Kiefer, J. E., and Weiss, G. H., 1987, Phys. Rev. A, 35, 1403.

[292] Feller, W., 1971, An Introduction to Probability Theory and Its Applications, Vol. II (New York: Wiley).

[293] Fischer, L., 1969, An Introduction to Gel Chromatography (Amsterdam: NorthHolland).

[294] Bottger, H., and Bryskin, V. V., 1980, Phil. Mag. B, 42, 297.

[295] van Lien, N., and ShklovskiI, B. I., 1981, Solid St. Commun., 38, 99.

[296] van der Mere, M., Schuchardt, R., and Keiper, R., 1982, Phys. Stat. Sol. B, 110, 571.

[297] Pandey, R. B., 1984, Phys. Rev. B, 30, 489.

[298] Dhar, D., 1984, J. Phys. A, 17, L257.

[299] Gefen, Y., and Goldhirsch, I., 1985, J. Phys. A, 18, L1037.

[300] Ohtsuki, T., 1982, J. phys. Soc. Japan, 51, 1493. 
[301] Seifert, E., and Suessenbach, M., 1984, J. Phys. A, 17, L703.

[302] Chowdhury, D., 1985, J. Phys. A, 18, L761.

[303] Stauffer, D., 1985, J. Phys. A, 18, 1827.

[304] de Arcangelis, L., Koplik, J., Redner, S., and Wilkinson, D., 1986, Phys. Rev. Lett., 57, 996.

[305] Redner, S., Koplik, J., and Wilkinson, D., 1987, J. Phys. A, 20, 1543.

[306] Roux, S., Mitescu, C., Charlaix, E., and Baudet, C., 1986, J. Phys. A, 19, L687.

[307] Barma, M., and Ransaswamy, R., 1986, J. Phys. A, 19, L605.

[308] Sahimi, M., Davis, H. T., and Scriven, L. E., 1983, Chem. Engng Commun., 23, 329.

[309] Michel, G., 1986, J. Phys. A, 19, 2461.

[310] Harder, H., Bunde, A., and Havlin, S., 1986, J. Phys. A, 19, L927.

[311] Laibowitz, R. G., and Gefen, Y., 1984, Phys. Rev. Lett., 53, 380.

[312] Luck, J. M., 1985, J. Phys. A, 18, 2061.

[313] Katz, A. J., and Thompson, A. H., 1985, Phys. Rev. Lett., 54, 1325.

[314] Avnir, D., Farin, D., and Pfeifer, P., 1984, Nature, 308, 261.

[315] Klein, U. K. A., and Haar, H. P., 1978, Chem. Phys. Lett., 58, 531.

[316] Kusumoto, Y., and Sato, H., 1979, Chem. Phys. Lett., 68, 13.

[317] Ediger, M. D., and FAYer, M. D., 1983, Macromolecules, 16, 1839.

[318] Klafter, J., and Blumen, A., 1985, J. Luminescence, 34, 77.

[319] Bunde, A., Havlin, S., Nossal, R., Stanley, H. E., and Weiss, G. H., 1985, J. chem. Phys., 83, 5909.

[320] Anlauf, J. K., 1986, Phys. Rev. Lett., 52, 1845.

[321] Agmon, N., and Glaser, M. L., 1986, Phys. Rev. A, 34, 656.

[322] Webman, I., 1984, Phys. Rev. Lett., 52, 220; 1984, J. statist. Phys., 26, 603.

[323] Klafter, J., Zumofen, G., and Blumen, A., 1984, J. Phys. Lett., Paris, 45, L49.

[324] Fixman, M., 1984, Phys. Rev. Lett., 53, 791.

[325] Havlin, S., Weiss, G. H., Kiefer, J. E., and Dishon, M., 1984, J. Phys. A, 17, L347.

[326] SHAPIR, Y.,

[327] Duordjevic, Z. B., 1986, Fractals in Physics, edited by L. Pietronero and E. Tosatti (Amsterdam: North Holland), p. 413; Redner, S., and Kang, K., 1984, J. Phys. A, 17, L451.

[328] Blumen, A., Klafter, J., and Zumofen, G., Phys. Rev. B, 28, 6112.

[329] Weiss, G. H., and Havlin, S., 1985, J. chem. Phys., 83, 5670.

[330] Meakin, P., and Stanley, H. E., 1984, J. Phys. A, 17, L173.

[331] Kang, K., and Redner, S., 1984, Phys. Rev. Lett., 52, 955.

[332] Torney, D. C., and McConnell, H. E., 1983, J. phys. Chem., 87, 1441; 1983, Proc. R. Soc., Lond. A, 387, 147.

[333] Kang, K., and Redner, S., 1985, Phys. Rev. A, 32, 435.

[334] Bramson, M., and Griffeath, D., 1980, Z. Wahrsceinlickeitstheori. verw. Gebiete, 53, 183.

[335] Kang, K., and Redner, S., 1984, Phys. Rev. A, 30, 2833.

[336] Peliti, L., J. Phys. A, 19, L365.

[337] von Smoluchowski, M. V., 1986, Z. Phys., 17, 557.

[338] Ben-Avraham, D., and Redner, S., 1986, Phys. Rev. A, 34, 501.

[339] Bunde, A., and Dieterich, W., 1984, Phys. Rev. B, 31, 6012.

[340] Kehr, K. W., and Binder, K., 1984, Applications of the Monte Carlo Methods in Statistical Physics, edited by K. Binder (Berlin: Springer).

[341] Sankey, D. F., and Fedders, P. H., 1977, Phys. Rev. B, 15, 3586.

[342] Fedders, P. A., 1978, Phys. Rev. B, 17, 40.

[343] Harris, T. E., 1965, J. appl. Prob., 2, 323.

[344] Levitt, D. G., 1973, Phys. Rev. A, 8, 3050.

[345] Richards, P. M., 1976, Phys. Rev. B, 16, 1363.

[346] Alexander, S., and Pincus, P., 1978, Phys. Rev. B, 18, 2011.

[347] van Beijeren, H., Kehr, K. W., and Kutner, R., 1983, Phys. Rev. B, 28, 5711.

[348] Bunde, A., Havlin, S., Nossal, R., and Stanley, H. E., 1985, Phys. Rev. B, 32, 3367.

[349] Bunde, A., Mosley, L. L., Stanley, H. E., Ben-Avraham, D., and Havlin, S., 1986, Phys. Rev. A, 34, 2575. 
[350] Amitrano, C., Bunde, A., and Stanley, H. E., 1985, J. Phys. A, 18, L923.

[351] Heupel, L., 1986, J. statist. Phys., 42, 541.

[352] Movshovitz, D., 1986, MSc thesis, Bar-Ilan University.

[353] Kremer, K., 1981, Z. Phys. B, 45, 148.

[354] Rammal, R., Toulouse, G., and Vannimenus, J., 1984, J. Phys., Paris, 45, 389.

[355] Harris, A. B., 1983, Z. Phys. B, 49, 347.

[356] Chakarabarti, B. K., and Kertesz, J., 1981, Z. Phys. B, 44, 221.

[357] Derrida, B., 1982, J. Phys. A, 15, L119.

[358] Ben-Avraham, D., and Havlin, S., 1984, Phys. Rev., 29, 2309.

[359] Klein, D. J., and Seitz, W. A., 1984, J. Phys., Paris, 45, L241.

[360] Kim, D., and Kahng, B., 1985, Phys. Rev. A, 31, 1193.

[361] Daoud, M., Cotton, J. P., Farnoux, B., Jannik, G., Sarma, G., Benoit, H., Duplessix, R., Picot, C., and De Gennes, P. G., 1975, Macromolecules, 8, 804.

[362] Domb, C., 1969, Adv. chem. Phys., 15, 229.

[363] Flory, P., 1971, Principles of Polymer Chemistry (Ithaca, NY: Cornell University Press).

[364] Nienhuis, B., 1982, Phys. Rev. Lett., 49, 1062.

[365] Webman, I., Jortner, J., and Cohen, M. H., 1977, Phys. Rev. B, 16, 2593.

[366] Coniglio, A., and Stanley, H. E., 1984, Phys. Rev. Lett., 52, 1068.

[367] Bunde, A., Coniglio, A., Hong, D. C., and Stanley, H. E., 1985, J. Phys. A, 18, L137.

[368] Havlin, S., Bunde, A., and Kiefer, J., 1986, J. Phys. A, 19, L419.

[369] Havlin, S., Bunde, A., and Stanley, H. E., 1986, Phys. Rev. B, 34, 445.

[370] Adler, J. Aharony, A., and Stauffer, D., 1985, J. Phys. A, 18, L129.

[371] Hong, D. C., Stanley, H. E., Coniglio, A., and Bunde, A., 1986, Phys. Rev. B, 33, 4565.

[372] Leyvraz, F., Adler, J., Aharony, A., Bunde, A., Coniglio, A., Hong, D. C., Stanley, H. E., and Stauffer, D., 1986, J. Phys. A, 19, 3683.

[373] Orr, B. G., Jaeger, H. M., and Goldman, A. M., 1985, Bull. Am. phys. Soc., 30, 232.

[374] Mannevil, P., and De Seze, L., 1985, Numerical Methods in the Study of Critical Phenomena, edited by I. Della Dora, J. Demongeot and B. Lacolle (Berlin: Springer).

[375] Herrmann, H. J., Landau, D. P., and Stauffer, D., 1982, Phys. Rev. Lett., 49, 412.

[376] Banavar, J. R., Haris, A. B., and Koplik, J., 1983, Phys. Rev. Lett., 51, 1115.

[377] Pandey, R. B., 1987, J. Phys. A, 20, L337.

[378] Ball, R., and Cates, M. E., 1984, J. Phys. A, 17, 2531.

[379] Chowdhury, D., and Chumrabarti, B. K., 1987, J. Phys. A, 18, L377.

[380] Manna, S. S., and Roy, A. K., 1987, Phys. Rev. A, 35, 4023.

[381] Helman, J. S., Coniglio, A., and Tsallis, C., 1984, Phys. Rev. Lett., 53, 1195; Cates, M. E., 1985, Ibid., 54, 1733; Stapelton, H. J., 1985, Ibid., 54, 1734; Helman, J. S., Coniglio, A., and Tsallis, C., 1985, Ibid., 54, 1735.

[382] Machta, J., and Moore, S. M., 1985, Phys. Rev. A, 32, 3164.

[383] Bug, A. L. R., and Gefen, Y., 1987, Phys. Rev. A, 35, 1301.

[384] Kerstein, A. R., and Pandey, R. B., 1987, Phys. Rev. A, 35, 3575.

[385] Grest, G. S., Webman, I., Safran, S. A., and Bug, A. L. R., 1986, Phys. Rev. A, 33, 2842.

[386] Kinzel, W., 1983, Percolation Structures Processes, edited by G. Deutscher, R. Zallen and J. Adler, Ann. Isr. phys. Soc., Vol. 5 (Bristol, UK: Adam Hilger).

[387] Vicsek, T., Kertesz, J., and Cserti, J., 1982, J. Phys. A, 15, L189.

[388] Stephen, M. J., 1981, J. Phys. C, 14, L1077.

[389] Hong, D., 1984, J. Phys. A, 17, L929.

[390] Khantha, M., and Stinchcombe, R. B., 1984, J. Phys. A, 20, 4951.

[391] Levine, D., and Steinhardt, P. J., 1984, Phys. Rev. Lett., 53.

[392] Pollak, M., 1978, The Metal Transition in Disordered Materials, edited by L. P. Friedman and D. P. Turnbull, 19th Scottish University Summer School in Physics, p. 84.

[393] Zyvagin, I. P., 1980, Phys. Stat. sol. (b), 101, 9.

[394] Bottger, H., and Bryskin, V. K., 1982, Phys. Stat. sol. (b), 113, 9. 
[395] Halperin, V., 1981, J. Phys., Paris, 42, 119.

[396] Halperin, V., 1985, Phil. Mag. B, 52, L75.

[397] Schmidlin, F. W., 1977, Phys. Rev. B, 16, 2362.

[398] Mitescu, C. D., 1983, Percolation Structures Processes, edited by G. Deutscher, R. Zallen and J. Adler, Ann. Isr. phys. Soc., Vol. 5 (Bristol, UK: Adam Hilger).

[399] Hoshen, J., and Kopelman, R., 1976, Phys. Rev. B, 14, 3428.

[400] Roy, A. K., and Chakrabarti, B. K., 1987, J. Phys. A, 20, 215.

[401] Lam, P. M., and Zhang, Z. Q., 1984, Z. Phys. B, 56, 155.

[402] Hoshen, J., and Kopelman, R., 1987, J. chem. Phys., 65, 2817.

[403] Banavar, J. R., Kadanoff, L., and Pruishen, A. M. M., 1985, Phys. Rev. B, 31, 1388.

[404] Argyrakis, P., and Kopelman, R., 1985, Phys. Rev. B, 31, 6008.

[405] Argyrakis, P., and Kopelman, R., 1986, J. chem. Phys., 84, 1047.

[406] Manna, S. S., and Chakrabarti, B. K., 1987, Phys. Rev. B, 36, 4078.

[407] Levy, Y. A., and Souillard, B., 1987, Europhys. Lett., 4, 233.

[408] Deutscher, G., Levy, Y., and Souillard, B., 1987, Europhys. Lett., 4, 577.

[409] Fourcade, B., and Tremblay, A.-M. S., 1987, Phys. Rev. A, 36, 2352.

[410] Platt, D. E., and Family, F., 1987, Phys. Rev. Lett., 58, 2786.

[411] Kahng, B., Batrouni, G. G., and Redner, S., 1987, J. Phys. A, 20, L827.

[412] Bunde, A., Harder, H., Havlin, S., and Roman, H. E., 1987, J. Phys., 20, L865.

[413] van Velzen, G. A., and Ernst, M. H., 1987, J. statist. Phys., 48, 677.

[414] Machta, J., et al., 1986, Phys. Rev. B, 33, 4818.

[415] Maloy, K. J., Feder, J., and Jossang, T., 1985, Phys. Rev. Lett., 55, 2688.

[416] Haus, J. W., and Kehr, K. W., 1987, Phys. Rev. B (to be published).

[417] Haus, J. W., Kehr, K. W., and Lyklema, J., 1982, Phys. Rev. B, 25, 2905.

[418] Nieuwenhuizen, Th. M., and Ernst, M. H., 1985, Phys. Rev. B, 31, 3518.

[419] Kim, G. O., OH, J. H., and Kim, J. J., 1987, J. Phys. A, 20, 1905.

[420] Koplik, J., Redner, S., and Wilkinson, D., 1987, J. Phys. A (to be published).

[421] Kopelman, R., Hoshen, J., Newhouse, J. S., and Argyrakis, P., 1983, J. statist. Phys., 30, 335.

[422] Lyo, S. K., 1987, Phys. Rev. B, 35, 7177.

[423] Harder, H., Bunde, A., and Dieterich, W., 1986, J. chem. Phys., 85, 4123.

[424] Borgan, Z., Elezovic, S., Knezevic, M., and Milosevic, S., 1987, J. Phys. A, 20, L715.

[425] Bradley, R. M., 1987, J. Phys. A, 20, L821.

[426] Dekeyser, R., Maritan, A., and Stella, A., 1987, Phys. Rev. A, 36, 2338.

[427] Harris, A. B., and Fisch, R., 1977, Phys. Rev. Lett., 38, 796. 